\title{
The supercharge and superconformal symmetry for $N=1$ supersymmetric quantum mechanics
}

\author{
T.E. Clark* , S.T. Love ${ }^{\dagger}$ and S.R. Nowling ${ }^{\ddagger}$ \\ Department of Physics, Purdue University, West Lafayette, IN 47907-1396
}

\begin{abstract}
The superspace Lagrangian formulation of $N=1$ supersymmetric quantum mechanics is presented. The general Lagrangian constructed out of chiral and antichiral supercoordinates containing up to two derivatives and with a canonically normalized kinetic energy term describes the motion of a nonrelativistic spin 1/2 particle with Landé $g$-factor 2 moving in two spatial dimensions under the influence of a static but spatially dependent magnetic field. Noether's theorem is derived for the general case and is used to construct superspace dependent charges whose lowest components give the superconformal generators. The supercoordinate of charges containing an $R$ symmetry charge, the supersymmetry charges and the Hamiltonian are combined to form a supercharge supercoordinate. Superconformal Ward identities for the quantum effective action are derived from the conservation equations and the source of potential symmetry breaking terms are identified.
\end{abstract}

\footnotetext{
*e-mail address: clark@physics.purdue.edu

$\dagger^{\dagger}$-mail address: love@physics.purdue.edu

†e-mail address: nowling@uiuc.edu
} 


\section{INTRODUCTION}

Supersymmetric quantum mechanics [1] has proven to be a fruitful testing ground for many of the intriguing consequences of the supersymmetry (SUSY) algebra which are also anticipated to be applicable in supersymmetric quantum field theories. Conversely, for those quantum mechanical models exhibiting a supersymmetry, the symmetry algebra can be exploited to extract many of the model spectral properties [2]. For example, the zero energy ground state wavefunctions for the 1 space dimension quantum mechanical systems whose dynamics is governed by the potential energy functions

$$
V(x)=\frac{1}{2} W^{2}(x) \pm \frac{1}{2} \frac{d W(x)}{d x},
$$

where $W(x)$ is an arbitary real function (superpotential), are given by

$$
\psi_{ \pm 0}(x)=N e^{\mp \int_{x_{0}}^{x} d x^{\prime} W\left(x^{\prime}\right)}
$$

where $N$ is a normalization constant. This result can be derived using the underlying $N=1$ supersymmetry exhibited by models of this type. The fact that models with such potential energy functions possess an underlying supersymmetry can be demonstrated in a variety of ways. One approach which manifestly incorporates the $N=1$ supersymmetry from the outset, entails the construction of a superspace consisting of the ordinary time coordinate, an anticommuting Grassmann variable and its conjugate. (See Appendix A for a detailed construction of such a superspace and its properties). The above class of models then emerges from the supersymmetric invariant action

$$
\Gamma_{0}=\int d V\left[\frac{1}{2} D \Phi \bar{D} \Phi-f(\Phi)\right]
$$

where $\Phi=\Phi^{\dagger}$ is a real supercoordinate whose $\theta \bar{\theta}$ component is $W(x)$ and after application of the equations of motion $\frac{d f(x)}{d x}=-W(x)$. The Hamiltonians for the two partner potential energy functions take the factorized form

$$
\begin{aligned}
H_{ \pm} & =-\frac{1}{2} \frac{d^{2}}{d x^{2}}+\frac{1}{2} W(x)^{2} \mp \frac{1}{2} \frac{d W(x)}{d x} \\
& =\frac{1}{\sqrt{2}}\left( \pm \frac{d}{d x}-W(x)\right) \frac{1}{\sqrt{2}}\left(\mp \frac{d}{d x}-W(x)\right) .
\end{aligned}
$$

The zero energy ground states satisfy $\frac{1}{\sqrt{2}}\left( \pm \frac{d}{d x}-W(x)\right) \psi_{ \pm 0}(x)=0$ whose solution is given above. 
Besides using real supercoordinates, the $N=1$ supersymmetry can also be realized in terms of chiral and antichiral supercoordinates. The purpose of this paper is to study the consequences of such realizations. In section 2, we present the most general supersymmetric action constructed out of such coordinates containing up to two derivatives. For a canonically normalized kinetic term, the model reduces to that describing a nonrelativistic spin $1 / 2$ particle with Landé $g$-factor 2 moving in 2 spatial dimensions under the influence of a static, but spatially dependent magnetic field.

The remainder of the paper is devoted to studying the space-time symmetry structure of the general $N=1$ supersymmetric action constructed out of the chiral and antichiral supercoordinates. The possible symmetries of the model are determined and the associated charges are constructed. The symmetry transformations are applied to the Green's functions and their (non-)conservation laws are expressed as Ward identities for the quantum effective action. The time translation and SUSY transformations of the supercoordinates are represented as superspace differential operators. The superspace action is shown to be invariant under these variations. The $N=1$ supersymmetry algebra and the construction of its representation on supercoordinates is presented in detail in Appendix A. There the methodology for constructing SUSY invariant actions is prescribed. The derivation of the superspace Noether's theorem for the construction of charges generating variations of the coordinates is presented in Section 3. The most general space-time symmetries of the model are found to be those of the $\operatorname{SPp}(2,1)$ superconformal group along with an additional $U(1)$ internal symmetry phase transformation. The Noether charge for each symmetry is constructed and the conservation laws derived. In general, the $U(1)$ and (super-)conformal symmetries are not conserved and the symmetry violating variation of the Lagrangian is presented. The charge (non-)conservation laws are used to derive the Ward identities obeyed by the generating functional for one-particle irreducible functions. The superconformal algebra is presented in Appendix B along with the representation of the symmetry transformations as superspace differential operator variations of the supercoordinates. The charges are shown to form the components of superspace multiplets which transform into each other under a restricted set of SUSY transformations. The multiplets are known as quasi-supercoordinates. Appendix $\mathrm{C}$ recalls the quantum action principle in superspace that underlies the derivation of the various Ward identities for the effective action, while in Appendix D, the spinning coordinate path integral is explicitly performed by evaluating the Grassmann determinant. So 
doing, the model is recast in terms of a matrix Hamiltonian which depends only on the particle ordinary spatial coordinates. Finally in Section 4, the multiplet that contains as its components a $R$-symmetry charge, the SUSY charges and the Hamiltonian is shown to form a supercoordinate referred to as the supercharge. All of the superconformal charges are constructed from these charges along with terms that are related to the global Weyl scaling charge for the chiral and antichiral supercoordinates. These are just the chiral, antichiral and total number operators. The Ward identity operators for the superconformal and $U(1)$ symmetries also form (quasi-)supercoordinates. The symmetry violating terms are determined for all the symmetry transformations. Only the trivial theory is both conformal and conformal SUSY invariant or simultaneously $U(1)$ and scale invariant. 


\section{2. $N=1$ SUPERSYMMETRY AND (ANTI-) CHIRAL SUPERCOORDINATES}

Manifestly supersymmetric quantum mechanical models are most conveniently formulated directly in superspace. The construction of this superspace can be achieved by augmenting the ordinary time variable by the introduction of an additional single complex Grassmann variable $\theta$, satisfying $\theta^{2}=0$, along with its complex conjugate $\bar{\theta}$, also satisfying $\bar{\theta}^{2}=0$. Together $(t, \theta, \bar{\theta})$ corresponds to a point in superspace. The detailed construction of this superspace along with the definition of SUSY covariant derivatives as well as real, chiral and anti-chiral supercoordinates are presented in Appendix A. In the following, we recall some of the salient features.

A chiral supercoordinate $\phi(t, \theta, \bar{\theta})$ and an antichiral supercoordinate $\bar{\phi}(t, \theta, \bar{\theta})$, have the component expansions

$$
\begin{aligned}
& \phi(t, \theta, \bar{\theta})=e^{-i \theta \bar{\theta} \partial_{t}}(z(t)+i \sqrt{2} \theta \xi(t))=z(t)+i \sqrt{2} \theta \xi(t)-i \theta \bar{\theta} \dot{z}(t) \\
& \bar{\phi}(t, \theta, \bar{\theta})=e^{i \theta \bar{\theta} \partial_{t}}(\bar{z}(t)-i \sqrt{2} \bar{\xi}(t) \bar{\theta})=\bar{z}(t)-i \sqrt{2} \bar{\xi}(t) \bar{\theta}+i \theta \bar{\theta} \dot{\bar{z}}(t)
\end{aligned}
$$

Here the components $z(t)$ and its complex conjugate $\bar{z}(t)$ are ordinary coordinates, while $\xi(t), \bar{\xi}(t)$ are Grassmann valued (spinning) coordinates. (Throughout the paper, a time derivative will be denoted by $\left.\dot{z}(t)=\partial_{t} z(t)=\frac{d}{d t} z(t)\right)$.

The most general SUSY invariant action through 2 derivatives may then be written in terms of the (anti-) chiral supercoordinates as

$$
\Gamma_{0}=\int d V \mathcal{L}=\int d V[K(\phi, \bar{\phi})+g(\phi, \bar{\phi}) D \phi \bar{D} \bar{\phi}]
$$

where the real Kähler prepotential supercoordinate $K=K(\phi, \bar{\phi})$ and real supercoordinate $g(\phi, \bar{\phi})$ are, in general, independent functions of the chiral and anti-chiral supercoordinates, $\phi$ and $\bar{\phi}$, but not there SUSY covariant derivatives. The nilpotent SUSY covariant derivatives are defined as $D=\frac{\partial}{\partial \theta}-i \bar{\theta} \frac{\partial}{\partial t}\left(D^{2}=0\right)$ and $\bar{D}=-\frac{\partial}{\partial \theta}+i \theta \frac{\partial}{\partial t}\left(\bar{D}^{2}=0\right)$. They anticommute to give the (SUSY covariant) time derivative $\{D, \bar{D}\}=2 i \frac{\partial}{\partial t}$. Performing the component decomposition yields the action

$$
\begin{aligned}
& \Gamma_{0}=\int d t L=\int d t\left\{4 g(z, \bar{z}) \dot{z} \dot{\bar{z}}+i\left(K_{z}(z, \bar{z}) \dot{z}-K_{\bar{z}}(z, \bar{z}) \dot{\bar{z}}\right)\right. \\
& +2 i g(z, \bar{z})(\dot{\xi} \overline{\bar{\xi}}-\dot{\xi} \bar{\xi})-K_{z \bar{z}}(z, \bar{z})[\xi, \bar{\xi}] \\
& \left.-i\left(g_{z}(z, \bar{z}) \dot{z}-g_{\bar{z}}(z, \bar{z}) \dot{\bar{z}}\right)[\xi, \bar{\xi}]\right\},
\end{aligned}
$$

Throughout the paper, subscripts indicate differentiation with respect to that variable so that, for example, $K_{z}=\frac{\partial K}{\partial z}, K_{z \bar{z}}=\frac{\partial^{2} K}{\partial z \partial \bar{z}}$. 
We first examine the above action when the kinetic energy term is canonically normalized which corresponds to taking $g(z, \bar{z})=\frac{1}{4}$ so the Lagrangian takes the form

$$
L=\dot{z} \dot{\bar{z}}+\frac{i}{2}(\xi \dot{\bar{\xi}}-\dot{\xi} \bar{\xi})+i\left(K_{z} \dot{z}-K_{\bar{z}} \dot{\bar{z}}\right)-K_{z \bar{z}}[\xi, \bar{\xi}]
$$

Under the supersymmetry transformations of the coordinates given by

$$
\begin{array}{rr}
i[Q, z]=\delta^{Q} z=i \sqrt{2} \xi & i\{Q, \xi\}=\delta^{Q} \xi=0 \\
i[Q, \bar{z}]=\delta^{Q} \bar{z}=0 & i\{Q, \bar{\xi}\}=\delta^{Q} \bar{\xi}=-\sqrt{2} \dot{\bar{z}} \\
i[\bar{Q}, z]=\delta^{\bar{Q}} z=0 & i\{\bar{Q}, \xi\}=\delta^{\bar{Q}} \xi=\sqrt{2} \dot{z} \\
i[\bar{Q}, \bar{z}]=\delta^{\bar{Q}} \bar{z}=-i \sqrt{2} \bar{\xi} & i\{\bar{Q}, \bar{\xi}\}=\delta^{\bar{Q}} \bar{\xi}=0,
\end{array}
$$

the Lagrangian transforms as a total time derivative

$$
\begin{aligned}
\delta^{Q} L & =\frac{d}{d t}\left(\frac{i}{\sqrt{2}} \dot{\bar{z}} \xi-\sqrt{2} K_{z} \xi\right) \\
\delta^{\bar{Q}} L & =\frac{d}{d t}\left(-\frac{i}{\sqrt{2}} \dot{z} \bar{\xi}-\sqrt{2} K_{\bar{z}} \bar{\xi}\right) .
\end{aligned}
$$

Consequently, the action, $\Gamma_{0}=\int d t L$, is SUSY invariant: $\delta^{Q} \Gamma_{0}=0=\delta^{\bar{Q}} \Gamma_{0}$.

Identifying the components $z(t)=\sqrt{\frac{m}{2}}(x(t)+i y(t))$ and $\bar{z}(t)=\sqrt{\frac{m}{2}}(x(t)-i y(t))$, where $x, y$ are the coordinates of 2-dimensional space, and rescaling the Kähler prepotential as $K \rightarrow q K$, where $q$ is the particle electric charge, this Lagrangian becomes

$$
L=\frac{1}{2} m \vec{v}^{2}+q \vec{A} \cdot \vec{v}+\frac{i}{2}(\xi \dot{\bar{\xi}}-\dot{\xi} \bar{\xi})+\frac{q}{2 m} B(x, y)[\xi, \bar{\xi}]
$$

where $\vec{v}=\hat{e}_{1} \dot{x}+\hat{e}_{2} \dot{y}$ is the particle velocity. In fact, this Lagrangian is recognized as one describing the planar motion of a nonrelativistic spin $1 / 2$ particle with Landé g-factor 2 moving under the action of a static, but spatially dependent magnetic field orientied perpendicular to the plane of motion: $\vec{B}=\hat{e}_{3} B(x, y)$, with

$$
B(x, y)=\epsilon_{i j} \partial_{i} A_{j}(x, y)=-\nabla^{2} K(x, y)
$$

and $A_{i}(x, y)$ the components of the vector potential. Thus the general supersymmetric quantum mechanical model in 2 spatial dimensions containing up to 2 derivatives and with canonically normalized kinetic energy term is simply that describing the motion of a nonrelativistic spin $1 / 2$ particle with $g$-factor 2 moving under the influence of a static, but spatially dependent magnetic field. 
The more familiar form for the corresponding Hamiltonian expressed solely in terms of the coordinates $x, y$ and their canonical momenta can be gleaned by integrating out the spinning variables as is explicitly performed in Appendix D. Alternatively, one could employ a specific representation using the Pauli matrices for the spinning coordinates: $\xi=\sigma_{+}$and $\bar{\xi}=\sigma_{-}$so that $\{\xi, \bar{\xi}\}=1$ and $[\xi, \bar{\xi}]=\sigma_{3}$. Here

$$
\sigma_{+}=\left(\begin{array}{cc}
0 & 1 \\
0 & 0
\end{array}\right) \quad \sigma_{-}=\left(\begin{array}{cc}
0 & 0 \\
1 & 0
\end{array}\right) \quad \sigma_{3}=\left(\begin{array}{cc}
1 & 0 \\
0 & -1
\end{array}\right)
$$

are the raising, lowering and third component Pauli matrices respectively. In either case, one secures the Lagrangian

$$
L=\frac{1}{2} m \vec{v}^{2}+q \vec{A} \cdot \vec{v}+\frac{q}{m} B(x, y) S,
$$

where $S=\frac{1}{2} \sigma_{3}$ is the spin operator. The corresponding Hamiltonian is then simply

$$
H=\frac{(\vec{p}-q \vec{A}(x, y))^{2}}{2 m}-\frac{q}{m} B(x, y) S,
$$

Note that the Landé $g$-factor is 2. As noted previously [3]- [6], this Hamiltonian is supersymmetric.

The complex, nilpotent $N=1$ supersymmetry charges, $Q$ and $\bar{Q}=(Q)^{\dagger}$, can be constructed as

$$
\begin{aligned}
& Q=\frac{i}{\sqrt{m}} \pi \sigma_{+} \\
& \bar{Q}=-\frac{i}{\sqrt{m}} \bar{\pi} \sigma_{-},
\end{aligned}
$$

where

$$
\begin{aligned}
& \pi \equiv\left(p_{x}-q A_{x}\right)-i\left(p_{y}-q A_{y}\right) \\
& \bar{\pi} \equiv\left(p_{x}-q A_{x}\right)+i\left(p_{y}-q A_{y}\right),
\end{aligned}
$$

have commutator

$$
[\bar{\pi}, \pi]=2 q B(x, y)
$$

These $Q, \bar{Q}$ nilpotent charges are seen to obey the supersymmetry algebra

$$
\{Q, Q\}=0=\{\bar{Q}, \bar{Q}\}
$$




$$
\begin{gathered}
\{Q, \bar{Q}\}=2 H \\
{[Q, H]=0=[\bar{Q}, H] .}
\end{gathered}
$$

The Hamiltonians for the two different spin projections form partners of this supersymmetric quantum mechanical system. The supersymmetry algebra was exploited [3] to explicitly construct the exact zero energy ground state wavefunction for the system. Modulo this ground state, the remainder of the eigenstates and eigenvalues of the two partner Hamiltonians form positive energy degenerate pairs [3].

For any supercoordinate $\phi(t, \theta, \bar{\theta})$, the supersymmetry transformations can be represented by superspace Grassmann derivatives (see Appendix A) as

$$
\begin{aligned}
\delta^{Q} \phi(t, \theta, \bar{\theta}) & =\left(\frac{\partial}{\partial \theta}+i \bar{\theta} \frac{\partial}{\partial t}\right) \phi(t, \theta, \bar{\theta}) \\
\delta^{\bar{Q}} \phi(t, \theta, \bar{\theta}) & =\left(-\frac{\partial}{\partial \bar{\theta}}-i \theta \frac{\partial}{\partial t}\right) \phi(t, \theta, \bar{\theta}) .
\end{aligned}
$$

The SUSY variations anticommute to yield a time derivative: $\left\{\delta^{Q}, \delta^{\bar{Q}}\right\}=-2 i \partial_{t}$. Since time translations are generated by time derivatives, this gives a representation of the SUSY algebra (2.16)

$$
\begin{gathered}
\left\{\delta^{Q}, \delta^{Q}\right\}=0=\left\{\delta^{\bar{Q}}, \delta^{\bar{Q}}\right\} \\
\left\{\delta^{Q}, \delta^{\bar{Q}}\right\}=-2 i \delta^{H} \\
{\left[\delta^{Q}, \delta^{H}\right]=0=\left[\delta^{\bar{Q}}, \delta^{H}\right]}
\end{gathered}
$$

where $\delta^{H} \phi(t, \theta, \bar{\theta})=\partial_{t} \phi(t, \theta, \bar{\theta})$.

In addition to time translation and SUSY invariance, the action of equation (2.3) also exhibits a symmetry under a $U(1)$ phase transformation of the (spinning) Grassmann coordinates given by $\xi^{\prime}=e^{i \rho} \xi$ and $\bar{\xi}^{\prime}=e^{-i \rho \bar{\xi}}$, where $\rho$ is a real parameter, while the coordinates $z$ and $\bar{z}$ are left invariant. Moreover, if $g$ and $K$ are functions only of the combination $\bar{\phi} \phi$, then the action is also invariant under rotations in the $x-y$ plane. This $U(1)$ phase transformation takes the form $\phi \rightarrow \phi^{\prime}=e^{i \alpha} \phi$ and $\bar{\phi} \rightarrow \bar{\phi}^{\prime}=e^{-i \alpha} \bar{\phi}$. Note that, in this case, it is the entire multiplet, including the ordinary and Grassmann odd coordinates, which participate in the rotation. These transformations are generated by superspace differential operators. Denoting the generator of the $U(1)$ transformation involving the entire supercoordinate by $J$ and the spinning coordinate only rotation by $R_{0}$, one finds

$$
\delta^{J} \phi(t, \theta, \bar{\theta})=i \phi(t, \theta, \bar{\theta}) \quad, \quad \delta^{J} \bar{\phi}(t, \theta, \bar{\theta})=-i \bar{\phi}(t, \theta, \bar{\theta})
$$


$\delta^{R_{0}} \phi(t, \theta, \bar{\theta})=i\left(\theta \frac{\partial}{\partial \theta}-\bar{\theta} \frac{\partial}{\partial \bar{\theta}}\right) \phi(t, \theta, \bar{\theta}) \quad, \quad \delta^{R_{0}} \bar{\phi}(t, \theta, \bar{\theta})=i\left(\theta \frac{\partial}{\partial \theta}-\bar{\theta} \frac{\partial}{\partial \bar{\theta}}\right) \bar{\phi}(t, \theta, \bar{\theta})$

The Lagrangian, and hence the action, is left unchanged by $R_{0}$ variations, $\delta^{R_{0}} \mathcal{L}=0$, but not, in general, by $J$-transformations, except, as stated above, when $g$ and $K$ are functions only of $\phi \bar{\phi}$.

As discussed in appendices $\mathrm{A}$ and $\mathrm{B}$, the $R$-transformations may also be defined to include a non-zero intrinsic $R$-weight, $n_{\phi}$, for the coordinates so that

$$
\begin{aligned}
& \delta^{R} \phi=i\left(n_{\phi}+\theta \frac{\partial}{\partial \theta}-\bar{\theta} \frac{\partial}{\partial \bar{\theta}}\right) \phi \\
& \delta^{R} \bar{\phi}=i\left(-n_{\phi}+\theta \frac{\partial}{\partial \theta}-\bar{\theta} \frac{\partial}{\partial \bar{\theta}}\right) \bar{\phi} .
\end{aligned}
$$

As we shall see, for a consistent embedding in the full superconformal algebra, it is required that $n_{\phi}=-2 d_{\phi}$, where $d_{\phi}$ is the the scaling weight of $\phi$. Note that the $R$-transformation with $n_{\phi}=0$ is $R_{0}$. Further note that since $\delta^{R}=n_{\phi} \delta^{J}+\delta^{R_{0}}$, in order for the action to be invariant under the general $R$-transformations, it must be $J$-invariant.

Throughout the paper, the transformations will be treated as functional differential operators or as derivatives acting in superspace. For a transformation $\mathcal{Q}$ with superspace differential operator representations $\delta^{\mathcal{Q}} \phi$ and $\delta^{\mathcal{Q}} \bar{\phi}$, the corresponding functional differential operator is defined as

$$
\delta^{\mathcal{Q}} \equiv \int d S \delta^{\mathcal{Q}} \phi \frac{\delta}{\delta \phi}+\int d \bar{S} \delta^{\mathcal{Q}} \bar{\phi} \frac{\delta}{\delta \bar{\phi}}
$$

So defined, the $\delta^{\mathcal{Q}}$ variation obeys the same algebra as the representation of the variation of the individual coordinates $\delta^{\mathcal{Q}} \phi$. The quantum action principle then relates the symmetry variations of the quantum effective action, $\Gamma$, to the transformations of the classical action $\Gamma_{0}$ (see Appendix C) as

$$
\delta^{\mathcal{Q}} \Gamma=\left[i \delta^{\mathcal{Q}} \Gamma_{0}\right] \Gamma=\left[i \int d V \delta^{\mathcal{Q}} \mathcal{L}\right] \Gamma
$$

where the right hand side is to be interpreted in terms of inserted one-particle irreducible (1-PI) functions. Thus, if the classical action is invariant so that $\delta^{\mathcal{Q}} \Gamma_{0}=\int d V \delta^{\mathcal{Q}} \mathcal{L}=0$, then so is the quantum effective action: $\delta^{\mathcal{Q}} \Gamma=0$. This is the $\mathcal{Q}$ symmetry Ward identitly for the generating functional of one-particle irreducible functions. (It should be noted that for certain highly singular potentials, a renormalization of the Green's functions may be 
required [7]. Further note that this procedure could potentially violate certain of the naive invariances of the classical action, a well known, yet anomalous, situation in field theory).

Since the classical action is SUSY and time translation invariant so is the quantum effective action

$$
\begin{aligned}
& \delta^{Q} \Gamma=0=\delta^{\bar{Q}} \Gamma \\
& \delta^{H} \Gamma=0 .
\end{aligned}
$$

Moreover, since the $R$-variations with zero $R$-weight are just total $\theta$ and $\bar{\theta}$ derivatives, $\delta^{R_{0}} \phi=i\left(\theta \frac{\partial}{\partial \theta}-\bar{\theta} \frac{\partial}{\partial \theta}\right) \phi$, the action is $R_{0}$-invariant. Hence

$$
\delta^{R_{0}} \Gamma=\left[i \int d V \delta^{R_{0}} \mathcal{L}\right] \Gamma=0,
$$

for $n_{\phi}=0=n_{\bar{\phi}}$. On the other hand, if $K$ and $g$ are not functions of $\bar{\phi} \phi$ only, then the action is not $J$ (or $R$ ) invariant but instead takes the form

$$
\delta^{J} \Gamma=\left[i \int d V \delta^{J} \mathcal{L}\right] \Gamma=\left[\int d V\left(\left(\bar{\phi} K_{\bar{\phi}}-\phi K_{\phi}\right)+\left(\bar{\phi} g_{\bar{\phi}}-\phi g_{\phi}\right) D \phi \bar{D} \bar{\phi}\right)\right] \Gamma .
$$




\section{NOETHER'S THEOREM AND SUPERCONFORMAL TRANSFORMATIONS}

A transformation of the coordinates corresponds to a good symmetry if the action remains invariant. In the present case, the action, $\Gamma_{0}$, is given as the integral over the real measure of the superspace Lagrangian as $\Gamma_{0}=\int d V \mathcal{L}$. Hence, the variation of the action is zero, $\delta \Gamma_{0}=\int d V \delta \mathcal{L}=0$, provided the Lagrangian transforms into the sum of chiral, $F$, and antichiral, $\bar{F}$, terms: $\delta \mathcal{L}=F+\bar{F}$. Note that since $\{D, \bar{D}\}=2 i \partial_{t}$ and $D^{2}=0=\bar{D}^{2}$, the case that the Lagrangian transforms into a total time derivative is subsummed by the above condition. Noether's theorem is obtained by considering the variation of the Lagrangian under the transformations of the supercoordinates, $\phi \rightarrow \phi+\delta \phi$ and $\bar{\phi} \rightarrow \bar{\phi}+\delta \bar{\phi}$, so that the Lagrangian transforms as

$$
\delta L=-\delta \phi \frac{1}{\bar{D}} \frac{\delta \Gamma_{0}}{\delta \phi}-\delta \bar{\phi} \frac{1}{D} \frac{\delta \Gamma_{0}}{\delta \bar{\phi}}+D\left(\delta \phi \frac{\partial \mathcal{L}}{\partial D \phi}\right)+\bar{D}\left(\delta \bar{\phi} \frac{\partial \mathcal{L}}{\partial \bar{D} \bar{\phi}}\right)
$$

where the Euler-Lagrange derivatives are given by

$$
\begin{aligned}
& \frac{\delta \Gamma_{0}}{\delta \phi}=-\bar{D}\left(\frac{\partial \mathcal{L}}{\partial \phi}-D\left(\frac{\partial \mathcal{L}}{\partial D \phi}\right)\right) \\
& \frac{\delta \Gamma_{0}}{\delta \bar{\phi}}=-D\left(\frac{\partial \mathcal{L}}{\partial \bar{\phi}}-\bar{D}\left(\frac{\partial \mathcal{L}}{\partial \bar{D} \bar{\phi}}\right)\right) .
\end{aligned}
$$

Here the $1 / D$ or $1 / \bar{D}$ operators in (3.1) are understood to cancel the corresponding $D$ or $\bar{D}$ derivatives in (3.2). Acting on equation (3.1) with $D$ and $\bar{D}$ yields

$$
\begin{aligned}
& D \delta \mathcal{L}=-\delta \bar{\phi} \frac{\delta \Gamma_{0}}{\delta \bar{\phi}}-(D \delta \bar{\phi})\left(\frac{1}{D} \frac{\delta \Gamma_{0}}{\delta \bar{\phi}}\right)+D\left(\bar{D}\left(\delta \bar{\phi} \frac{\partial \mathcal{L}}{\partial \bar{D} \bar{\phi}}\right)-\delta \phi\left(\frac{1}{\bar{D}} \frac{\delta \Gamma_{0}}{\delta \phi}\right)\right) \\
& \bar{D} \delta \mathcal{L}=-\delta \phi \frac{\delta \Gamma_{0}}{\delta \phi}-(\bar{D} \delta \phi)\left(\frac{1}{\bar{D}} \frac{\delta \Gamma_{0}}{\delta \phi}\right)+\bar{D}\left(D\left(\delta \phi \frac{\partial \mathcal{L}}{\partial D \phi}\right)-\delta \bar{\phi}\left(\frac{1}{D} \frac{\delta \Gamma_{0}}{\delta \bar{\phi}}\right)\right) .
\end{aligned}
$$

These identities lead to the superspace Noether's theorem. The Noether charge, $\mathcal{Q}$, corresponding to this transformation is defined as

$$
\mathcal{Q} \equiv \frac{1}{2}\left\{\bar{D}\left(\delta \bar{\phi} \frac{\partial \mathcal{L}}{\partial \bar{D} \bar{\phi}}\right)-\delta \phi\left(\frac{1}{\bar{D}} \frac{\delta \Gamma_{0}}{\delta \phi}\right)-D\left(\delta \phi \frac{\partial \mathcal{L}}{\partial D \phi}\right)+\delta \bar{\phi}\left(\frac{1}{D} \frac{\delta \Gamma_{0}}{\delta \bar{\phi}}\right)\right\}
$$

It follows that the SUSY covariant derivatives of the charge are

$$
\begin{aligned}
& D \mathcal{Q}=\delta \bar{\phi} \frac{\delta \Gamma_{0}}{\delta \bar{\phi}}+\frac{1}{2} D \delta \mathcal{L}+(D \delta \bar{\phi})\left(\frac{1}{D} \frac{\delta \Gamma_{0}}{\delta \bar{\phi}}\right) \\
& \bar{D} \mathcal{Q}=-\delta \phi \frac{\delta \Gamma_{0}}{\delta \phi}-\frac{1}{2} \bar{D} \delta \mathcal{L}-(\bar{D} \delta \phi)\left(\frac{1}{\bar{D}} \frac{\delta \Gamma_{0}}{\delta \phi}\right) .
\end{aligned}
$$


Further differentiation leads to the local form of Noether's theorem

$$
\begin{aligned}
\{D, \bar{D}\} \mathcal{Q}= & 2 i \frac{\partial}{\partial t} \mathcal{Q} \\
= & \bar{D}\left(\delta \bar{\phi} \frac{\delta \Gamma_{0}}{\delta \bar{\phi}}+(D \delta \bar{\phi}) \frac{1}{D} \frac{\delta \Gamma_{0}}{\delta \bar{\phi}}\right) \\
& \quad-D\left(\delta \phi \frac{\delta \Gamma_{0}}{\delta \phi}+(\bar{D} \delta \phi) \frac{1}{\bar{D}} \frac{\delta \Gamma_{0}}{\delta \phi}\right)-\frac{1}{2}[D, \bar{D}] \delta \mathcal{L}
\end{aligned}
$$

Inserting this in the one-particle irreducible functions, applying the equations of motion for the Green's functions (see Appendix C) and integrating over the time while ignoring total time derivatives, the Ward identity for the variation of the effective action is secured in the form of the quantum action principle

$$
\begin{aligned}
\delta \Gamma & \equiv\left(\int d S \delta \phi \frac{\delta}{\delta \phi}+\int d \bar{S} \delta \bar{\phi} \frac{\delta}{\delta \bar{\phi}}\right) \Gamma \\
& =\left[i \int d V \delta \mathcal{L}\right] \Gamma-\left[i \int d S(\bar{D} \delta \phi) \frac{1}{\bar{D}} \frac{\delta \Gamma_{0}}{\delta \phi}\right] \Gamma-\left[i \int d \bar{S}(D \delta \bar{\phi}) \frac{1}{D} \frac{\delta \Gamma_{0}}{\delta \bar{\phi}}\right] \Gamma,
\end{aligned}
$$

with $\Gamma$ the quantum effective action (the one-particle irreducible function generating functional). In obtaining this result, we have used that $\int d S \chi=\int d t d \theta \chi=\int d t D \chi$ and $\int d \bar{S} \bar{\chi}=\int d t d \bar{\theta} \bar{\chi}=-\int d t \bar{D} \bar{\chi}$, with $\chi$ and $\bar{\chi}$ Grassmann odd functions of the supercoordinates and their SUSY covariant derivatives.

We now focus on transformations which correspond to a reparametrization of superspace:

$$
\begin{aligned}
& t \rightarrow t^{\prime}(t, \theta, \bar{\theta}) \\
& \theta \rightarrow \theta^{\prime}(t, \theta, \bar{\theta}) \\
& \bar{\theta} \rightarrow \bar{\theta}^{\prime}(t, \theta, \bar{\theta})
\end{aligned}
$$

Note that the variations of the superspace coordinates are general functions of superspace. Under such transformations, the chiral and antichiral supercoordinates transform as

$$
\begin{aligned}
\phi(t, \theta, \bar{\theta}) & \rightarrow e^{i \Lambda\left(t^{-1}, \theta^{-1}, \bar{\theta}^{-1}\right)} \phi\left(t^{-1}, \theta^{-1}, \bar{\theta}^{-1}\right) \\
\bar{\phi}(t, \theta, \bar{\theta}) & \rightarrow e^{-i \bar{\Lambda}\left(t^{-1}, \theta^{-1}, \bar{\theta}^{-1}\right)} \bar{\phi}\left(t^{-1}, \theta^{-1}, \bar{\theta}^{-1}\right),
\end{aligned}
$$

where $\Lambda$ and $\bar{\Lambda}$ are complex independent functions of superspace and the inverse transformation refers to the reparametrization given by equation (3.8) where the primed variables are to be replaced with unprimed ones. For infinitesimal parameters, the reparametrization takes the form

$$
t \rightarrow t+\delta t(t, \theta, \bar{\theta})
$$




$$
\begin{aligned}
& \theta \rightarrow \theta+\delta \theta(t, \theta, \bar{\theta}) \\
& \bar{\theta} \rightarrow \bar{\theta}+\delta \bar{\theta}(t, \theta, \bar{\theta})
\end{aligned}
$$

while the chiral and anti-chiral supercoordinates vary as

$$
\begin{aligned}
& \phi(t, \theta, \bar{\theta}) \rightarrow \phi(t, \theta, \bar{\theta})+\delta \phi(t, \theta, \bar{\theta})=(1+i \delta \Lambda) \phi(t-\delta t, \theta-\delta \theta, \bar{\theta}-\delta \bar{\theta}) \\
& \bar{\phi}(t, \theta, \bar{\theta}) \rightarrow \bar{\phi}(t, \theta, \bar{\theta})+\delta \bar{\phi}(t, \theta, \bar{\theta})=(1+i \delta \bar{\Lambda}) \bar{\phi}(t-\delta t, \theta-\delta \theta, \bar{\theta}-\delta \bar{\theta})
\end{aligned}
$$

where $\delta \Lambda$ and $\delta \bar{\Lambda}$ are infinitesimal complex independent functions of superspace. Retaining terms through linear in the small variations gives

$$
\begin{aligned}
\delta \phi & =\left(i \delta \Lambda-\delta t \partial_{t}-\delta \theta \partial_{\theta}-\delta \bar{\theta} \partial_{\bar{\theta}}\right) \phi \\
& =\left(i \delta \Lambda-\delta B \partial_{t}-\delta \theta D\right) \phi \\
\delta \bar{\phi} & =\left(-i \delta \bar{\Lambda}-\delta t \partial_{t}-\delta \theta \partial_{\theta}-\delta \bar{\theta} \partial_{\bar{\theta}}\right) \bar{\phi} \\
& =\left(-i \delta \bar{\Lambda}-\delta B \partial_{t}+\delta \bar{\theta} \bar{D}\right) \bar{\phi},
\end{aligned}
$$

where $\delta B \equiv \delta t+i \delta \theta \bar{\theta}+i \delta \bar{\theta} \theta$.

Under this reparametrization, the terms in the superspace Lagrangian, $\mathcal{L}=K+g D \phi \bar{D} \bar{\phi}$, transform as

$$
\begin{aligned}
\delta(g D \phi \bar{D} \bar{\phi})= & i g \phi \bar{\phi}(\bar{D} D \delta \Lambda+D \bar{D} \delta \bar{\Lambda}) \\
& +i(\delta \Lambda-\delta \bar{\Lambda}+i(D \delta \theta)-i(\bar{D} \delta \bar{\phi})) g D \phi \bar{D} \bar{\phi} \\
& -i g \bar{D}(D \delta \Lambda \phi \bar{\phi})-i g D(\bar{D} \delta \bar{\Lambda} \phi \bar{\phi})+\bar{D}(\delta B g D \phi \dot{\bar{\phi}})-D(\delta B g \dot{\phi} \bar{D} \bar{\phi}) \\
& +\frac{i}{2}(\delta \Lambda-\delta \bar{\Lambda})\left(\phi g_{\phi}+\bar{\phi} g_{\bar{\phi}}\right) D \phi \bar{D} \bar{\phi}+\frac{i}{2}(\delta \Lambda+\delta \bar{\Lambda})\left(\phi g_{\phi}-\bar{\phi} g_{\bar{\phi}}\right) D \phi \bar{D} \bar{\phi} \\
\delta K= & \frac{i}{2}(\delta \Lambda-\delta \bar{\Lambda})\left(\phi K_{\phi}+\bar{\phi} K_{\bar{\phi}}\right)+\frac{i}{2}(\delta \Lambda+\delta \bar{\Lambda})\left(\phi K_{\phi}-\bar{\phi} K_{\bar{\phi}}\right) \\
& +\frac{i}{2} D(\delta B \bar{D} K)+\frac{i}{2} \bar{D}(\delta B D K) \\
& -\left(\delta \theta+\frac{i}{2} \bar{D} \delta B\right) D K+\left(\delta \bar{\theta}-\frac{i}{2} D \delta B\right) \bar{D} K
\end{aligned}
$$

while the Noether charge corresponding to these variations has the form

$$
\begin{aligned}
\mathcal{Q}= & \frac{1}{2}\left\{\frac{i}{2}(\delta \Lambda+\delta \bar{\Lambda})\left(\phi K_{\phi}+\bar{\phi} K_{\bar{\phi}}\right)+\frac{i}{2}(\delta \Lambda-\delta \bar{\Lambda})\left(\phi K_{\phi}-\bar{\phi} K_{\bar{\phi}}\right)\right. \\
& -\delta B\left(\dot{\phi} K_{\phi}-\dot{\bar{\phi}} K_{\bar{\phi}}\right) \\
& -\delta \theta D K-\delta \bar{\theta} \bar{D} K+D(\delta \theta g D \phi \bar{D} \bar{\phi})+\delta \theta g D \phi D \bar{D} \bar{\phi}+\bar{D}(\delta \bar{\theta} g D \phi \bar{D} \bar{\phi})-\delta \bar{\theta} g \bar{D} D \phi \bar{D} \bar{\phi} \\
& -i D(\Lambda g \phi \bar{D} \bar{\phi})-i \Lambda g \phi D \bar{D} \bar{\phi}+i \bar{D}(\bar{\Lambda} g D \phi \bar{\phi})+i \bar{\Lambda} g \bar{D} D \phi \bar{\phi}
\end{aligned}
$$




$$
+D(\delta B g \dot{\phi} \bar{D} \bar{\phi})+\delta B g \dot{\phi} D \bar{D} \bar{\phi}+\bar{D}(\delta B g D \phi \dot{\bar{\phi}})+\delta B g \bar{D} D \phi \dot{\bar{\phi}}\}
$$

The charge generates the intrinsic variations of the coordinates as $i[\mathcal{Q}, \phi]=\delta^{\mathcal{Q}} \phi$. That is, it gives the change in the operator which results in order to insure the invariance of their matrix elements when compared in the two frames of reference. From this it follows that the variation of a derivative of the supercoordinate is the derivative of the variation of the supercoordinate, e.g. $\delta^{\mathcal{Q}} D \phi=D \delta^{\mathcal{Q}} \phi$. We need to insure that the symmetry charges do not change the chirality of the supercoordinates. This chirality consistency requirement further restricts the transformation parameters. The chiral constraint, $\bar{D} \delta \phi=0$, dictates that

$$
\begin{aligned}
\bar{D} \delta \Lambda & =0 \\
\bar{D} \delta \theta & =0 \\
\bar{D} \delta B & =2 i \delta \theta,
\end{aligned}
$$

while the antichiral constraint $D \delta \bar{\phi}=0$ mandates

$$
\begin{aligned}
D \delta \bar{\Lambda} & =0 \\
D \delta \bar{\theta} & =0 \\
D \delta B & =-2 i \delta \bar{\theta} .
\end{aligned}
$$

The solutions to these equations require the transformation parameters $\delta \Lambda, \quad \delta \theta \equiv-i \delta X$ $(\delta \bar{\Lambda}, \quad \delta \bar{\theta} \equiv i \delta \bar{X})$ to be chiral (antichiral) functions of superspace. Note that $\delta X$ and $\delta \bar{X}$ are Grassmann odd. In addition, the equations for $\delta B$ are satisfied provided

$$
\begin{aligned}
\delta t & =\delta A+\delta X \bar{\theta}+\delta \bar{X} \theta \\
& =\delta \bar{A}-\delta X \bar{\theta}-\delta \bar{X} \theta \\
& =\frac{1}{2}(\delta A+\delta \bar{A})
\end{aligned}
$$

where $\delta A(\delta \bar{A})$ are chiral (antichiral): $\bar{D} \delta A=0(D \delta \bar{A}=0)$.

Noting that the chirality of the supercoordinate variations (3.16) and (3.17) implies that $\delta \theta+\frac{i}{2} \bar{D} B=0=\delta \bar{\theta}-\frac{i}{2} D B$, it follows that the variation of the Kähler potential simplifies to

$$
\begin{aligned}
& \delta K=\frac{i}{2}(\delta \Lambda-\delta \bar{\Lambda})\left(\phi K_{\phi}+\bar{\phi} K_{\bar{\phi}}\right)+\frac{i}{2}(\delta \Lambda+\delta \bar{\Lambda})\left(\phi K_{\phi}-\bar{\phi} K_{\bar{\phi}}\right) \\
& +\frac{i}{2} D(\delta B \bar{D} K)+\frac{i}{2} \bar{D}(\delta B D K) \text {. }
\end{aligned}
$$


Our goal is secure the form of the various parameters characterizing the superspace reparametrization which leads to maximum possible symmetries of the action without restriction to the specific forms of the $g$ and $K$ real superfunctions. To accomplish this, we need to further restrict the parameters so that the variation of the term containing $g$, Eq. (3.13,) has an analogous structure to the term containing $K$, Eq. (3.19). This is achieved by requiring

$$
\begin{gathered}
\frac{\partial}{\partial t}(\delta \Lambda+\delta \bar{\Lambda})=0 \\
\delta \Lambda-\delta \bar{\Lambda}+D \delta X+\bar{D} \delta \bar{X}=0 .
\end{gathered}
$$

The resultant variation of the term containing $g$ now becomes

$$
\begin{aligned}
\delta[g D \phi \bar{D} \bar{\phi}]= & \frac{i}{2}(\delta \Lambda-\delta \bar{\Lambda})\left(\phi g_{\phi}+\bar{\phi} g_{\bar{\phi}}\right) D \phi \bar{D} \bar{\phi} \\
& +\frac{i}{2}(\delta \Lambda+\delta \bar{\Lambda})\left(\phi g_{\phi}-\bar{\phi} g_{\bar{\phi}}\right) D \phi \bar{D} \bar{\phi} \\
& +\bar{D}(\delta B g D \phi \dot{\bar{\phi}})-i g \bar{D}(D \delta \Lambda \phi \bar{\phi}) \\
& -D(\delta B g \dot{\phi} \bar{D} \bar{\phi})-i g D(\bar{D} \delta \bar{\Lambda} \phi \bar{\phi}) .
\end{aligned}
$$

Solving equations (3.20) for the transformation parameters gives

$$
\begin{aligned}
\delta \Lambda & =\frac{1}{2} \delta \alpha+\frac{i}{2} \delta \beta+\frac{i}{2} \delta \gamma t+\theta \delta \lambda+\frac{1}{2} \theta \bar{\theta} \delta \gamma \\
\delta \bar{\Lambda} & =\frac{1}{2} \delta \alpha-\frac{i}{2} \delta \beta-\frac{i}{2} \delta \gamma t+\bar{\theta} \delta \bar{\lambda}+\frac{1}{2} \theta \bar{\theta} \delta \gamma \\
\delta X & =\delta \eta+\frac{i}{2} \delta \bar{\lambda} t+\theta\left(\frac{1}{2} \delta \rho-\frac{i}{2} \delta \beta-\frac{i}{2} \delta \gamma t\right)+\frac{1}{2} \theta \bar{\theta} \delta \bar{\lambda} \\
\delta \bar{X} & =\delta \bar{\eta}+\frac{i}{2} \delta \lambda t+\bar{\theta}\left(\frac{1}{2} \delta \rho+\frac{i}{2} \delta \beta+\frac{i}{2} \delta \gamma t\right)-\frac{1}{2} \theta \bar{\theta} \delta \bar{\lambda} \\
\delta A & =\delta \epsilon-\delta \beta t-\frac{1}{2} \delta \gamma t^{2}+\theta(2 \delta \bar{\eta}+i \delta \lambda t)+i \theta \bar{\theta}(\delta \beta+\delta \gamma t) \\
\delta \bar{A} & =\delta \epsilon-\delta \beta t-\frac{1}{2} \delta \gamma t^{2}-\bar{\theta}(2 \delta \eta+i \delta \bar{\lambda} t)-i \theta \bar{\theta}(\delta \beta+\delta \gamma t),
\end{aligned}
$$

where $\delta \alpha, \delta \beta, \delta \gamma, \delta \rho, \delta \epsilon$ are infinitesimal real constants and $\delta \eta, \delta \bar{\eta}, \delta \lambda, \delta \bar{\lambda}$ are infinitesimal complex constant anticommuting parameters. Recall that

$$
\delta B=\delta t+i \delta \theta \bar{\theta}+i \delta \bar{\theta} \theta=\frac{1}{2}(\delta A+\delta \bar{A})+\delta X \bar{\theta}-\delta \bar{X} \theta,
$$

while the superspace infinitesimal transformation parameters are

$$
\begin{aligned}
& \delta t=\frac{1}{2}(\delta A+\delta \bar{A})=\delta \epsilon-\delta \beta t-\frac{1}{2} \delta \gamma t^{2}+\theta\left(\delta \bar{\eta}+\frac{i}{2} \delta \lambda t\right)-\bar{\theta}\left(\delta \eta+\frac{i}{2} \delta \bar{\lambda} t\right) \\
& \delta \theta=-i \delta X=-i\left(\delta \eta+\frac{i}{2} \delta \bar{\lambda} t+\theta\left(\frac{1}{2} \delta \rho-\frac{i}{2} \delta \beta-\frac{i}{2} \delta \gamma t\right)+\frac{1}{2} \theta \bar{\theta} \delta \bar{\lambda}\right)
\end{aligned}
$$




$$
\delta \bar{\theta}=i \delta \bar{X}=i\left(\delta \bar{\eta}+\frac{i}{2} \delta \lambda t+\bar{\theta}\left(\frac{1}{2} \delta \rho+\frac{i}{2} \delta \beta+\frac{i}{2} \delta \gamma t\right)-\frac{1}{2} \theta \bar{\theta} \delta \bar{\lambda}\right)
$$

Taken together, these are recognized as the class of infinitesimal superconformal transformations along with an additional overall $U_{J}(1)$ phase transformation of the coordinates. To obtain the finite superconformal transformations, one simply integrates these equations yielding (with the corresponding finite parameters)

$$
\begin{aligned}
t^{\prime} & =\frac{e^{-\beta} t+\epsilon+\theta \bar{\eta}-\bar{\theta} \eta}{1+\frac{1}{2} \gamma t-\frac{i}{2} \theta \lambda+\frac{i}{2} \bar{\theta} \bar{\lambda}} \\
\theta^{\prime} & =\frac{e^{-\frac{i}{2} \rho-\frac{1}{2} \beta} \theta-i \eta+\frac{1}{2} \bar{\lambda} t}{1+\frac{1}{2} \gamma t-\frac{i}{2} \theta \lambda+\frac{i}{2} \bar{\theta} \bar{\lambda}} \\
\bar{\theta}^{\prime} & =\frac{e^{+\frac{i}{2} \rho-\frac{1}{2} \beta \bar{\theta}}+i \bar{\eta}-\frac{1}{2} \lambda t}{1+\frac{1}{2} \gamma t-\frac{i}{2} \theta \lambda+\frac{i}{2} \bar{\theta} \bar{\lambda}} .
\end{aligned}
$$

Grouping terms with common variation parameters, the infinitesimal superconformal and $U_{J}(1)$ phase transformations of the supercoordinates take the form

$$
\begin{aligned}
\delta \phi= & \frac{i}{2} \delta \alpha \phi+\delta \beta\left(-\frac{1}{2}+t \partial_{t}+\frac{1}{2} \theta D\right) \phi+\frac{i}{2} \delta \rho\left(\theta D+2 i \theta \bar{\theta} \partial_{t}\right) \phi \\
& +\frac{1}{2} \delta \gamma\left(-t+i \theta \bar{\theta}+t^{2} \partial_{t}+t \theta D\right) \phi-\delta \epsilon \partial_{t} \phi \\
& +i \delta \eta\left(D+2 i \bar{\theta} \partial_{t}\right) \phi+\delta \bar{\eta} 2 \theta \partial_{t} \phi \\
& -i \delta \lambda \theta\left(1-t \partial_{t}\right) \phi-\frac{1}{2} \delta \bar{\lambda}\left(t D-i \theta \bar{\theta} D+2 i \bar{\theta} t \partial_{t}\right) \phi \\
\delta \bar{\phi}= & -\frac{i}{2} \delta \alpha \bar{\phi}+\delta \beta\left(-\frac{1}{2}+t \partial_{t}-\frac{1}{2} \bar{\theta} \bar{D}\right) \bar{\phi}+\frac{i}{2} \delta \rho\left(\bar{\theta} \bar{D}+2 i \theta \bar{\theta} \partial_{t}\right) \bar{\phi} \\
& +\frac{1}{2} \delta \gamma\left(-t-i \theta \bar{\theta}+t^{2} \partial_{t}-t \bar{\theta} \bar{D}\right) \bar{\phi}-\delta \epsilon \partial_{t} \bar{\phi} \\
& +i \delta \bar{\eta}\left(\bar{D}-2 i \theta \partial_{t}\right) \bar{\phi}-\delta \eta 2 \bar{\theta} \partial_{t} \bar{\phi} \\
& +i \delta \bar{\lambda} \bar{\theta}\left(1-t \partial_{t}\right) \bar{\phi}-\frac{1}{2} \delta \lambda\left(t \bar{D}+i \theta \bar{\theta} \bar{D}-2 i \theta t \partial_{t}\right) \bar{\phi} .
\end{aligned}
$$

Note that these are identical to the superconformal and $U_{J}(1)$ phase transformations given

in Appendix B corresponding to the case where $d_{\phi}=d_{\bar{\phi}}=-\frac{1}{2}$ and $n_{\phi}=-2 d_{\phi}=-n_{\bar{\phi}}=$ 1. It follows that the largest possible symmetry group is just the direct product group $O S p(2,1) \otimes U_{J}(1)$.

Since the supercoordinate Lagrangian $\mathcal{L}=K+g D \phi \bar{D} \bar{\phi}$ is the most general (through two derivatives) SUSY and time translationally invariant object constructed from the chiral supercoordinates, the corresponding action is, in general, not invariant under these more restrictive superconformal transformations. Mutatis mutandis, the SUSY (and time translation) Ward identities $\delta^{Q} \Gamma=0=\delta^{\bar{Q}} \Gamma$ are functional differential equations for the quantum 
effective action whose solution (through two derivatives ) determines the classical action, $\Gamma_{0}=\int d V \mathcal{L}$ to be given by $\mathcal{L}=K+g D \phi \bar{D} \bar{\phi}$ with arbitrary $K$ and $g$. Only for specific forms of $g$ and $K$ will this larger symmetry be manifest. Isolating the $U_{J}(1)$ transformation by taking $\delta \Lambda=\delta \bar{\Lambda}=\delta \alpha / 2$, it follows from equations (3.19) and (3.21) that $\delta^{J} \Gamma=0$ provided $K$ and $g$ satisfy $K_{z} z-K_{\bar{z}} \bar{z}=0=g_{z} z-g_{\bar{z}} \bar{z}$. As such, $g(\phi, \bar{\phi})$ and $K(\phi, \bar{\phi})$ are functions only of $\phi \bar{\phi}$. On the other hand, isolating the dilataion transformation by choosing the parameters as $\delta \Lambda=-\delta \bar{\Lambda}=i \delta \beta / 2$, dilatation invariance, $\delta^{D} \Gamma=0$ results provided $K$ and $g$ are scale independent. This follows, using equations (3.19) and (3.21), provided $K_{z} z+K_{\bar{z}} \bar{z}=0=g_{z} z+g_{\bar{z}} \bar{z}$ which, in turn, dictates that $g(\phi, \bar{\phi})$ and $K(\phi, \bar{\phi})$ must be are functions of $\phi / \bar{\phi}$. When the dilatation charge is conserved, the commutation relation $[H, D]=i H$ implies that $e^{i \beta D} H e^{-i \beta D}=e^{\beta} H$ for any real $\beta$. Thus if $\mid E>$ is an eigenstate of $H, H|E>=E| E>$, then $e^{-i \beta D} \mid E>$ is also an energy eigenstate with energy eigenvalue $e^{\beta} E$ for any real $\beta$. Consequently, the spectrum of $H$ consists of all non- negative real numbers with the ground state having zero energy. All excited states are doubly degenerate as a consequence of the SUSY. Finally, we note that requiring both $U_{J}(1)$ and dilatation invariance leads to the free theory only.

The variation of the Lagrangian is given by the sum of equations (3.19) and (3.21) and can be written as

$$
\begin{aligned}
\delta \mathcal{L}= & F+\bar{F}+\frac{i}{2}(\delta \Lambda-\delta \bar{\Lambda})\left(\left(\phi K_{\phi}+\bar{\phi} K_{\bar{\phi}}\right)+\left(\phi g_{\phi}+\bar{\phi} g_{\bar{\phi}}\right) D \phi \bar{D} \bar{\phi}\right) \\
& +\frac{i}{2}(\delta \Lambda+\delta \bar{\Lambda})\left(\left(\phi K_{\phi}-\bar{\phi} K_{\bar{\phi}}\right)+\left(\phi g_{\phi}-\bar{\phi} g_{\bar{\phi}}\right) D \phi \bar{D} \bar{\phi}\right) \\
& +\frac{i}{2} \bar{D}(\delta \Lambda-\delta \bar{\Lambda})\left(\phi g_{\phi}+\bar{\phi} g_{\bar{\phi}}\right) D \phi \bar{\phi}-\frac{i}{2} \bar{D}(\delta \Lambda+\delta \bar{\Lambda})\left(\phi g_{\phi}-\bar{\phi} g_{\bar{\phi}}\right) D \phi \bar{\phi} \\
& -\frac{i}{2} D(\delta \Lambda-\delta \bar{\Lambda})\left(\phi g_{\phi}+\bar{\phi} g_{\bar{\phi}}\right) \phi \bar{D} \bar{\phi}+\frac{i}{2} D(\delta \Lambda+\delta \bar{\Lambda})\left(\phi g_{\phi}-\bar{\phi} g_{\bar{\phi}}\right) \phi \bar{D} \bar{\phi}
\end{aligned}
$$

where the chiral, $F$, and antichiral, $\bar{F}$, combinations are defined by

$$
\begin{aligned}
F & =\frac{i}{2} \bar{D}(\delta B D K)-i \bar{D}(D \delta \Lambda g \phi \bar{\phi})+\bar{D}(\delta B g D \phi \dot{\bar{\phi}}) \\
\bar{F} & =\frac{i}{2} D(\delta B \bar{D} K)-i D(\bar{D} \delta \bar{\Lambda} g \phi \bar{\phi})-D(\delta B g D \dot{\phi} \bar{D} \bar{\phi})
\end{aligned}
$$

Note that the last two terms on the right hand side of equation (3.27) can be written as $-i D \delta \Lambda Y-i \bar{D} \delta \bar{\Lambda} \bar{Y}$ where

$$
\begin{aligned}
Y & =\bar{\phi} g_{\bar{\phi}} \phi \bar{D} \bar{\phi} \\
\bar{Y} & =\phi g_{\phi} D \phi \bar{\phi} .
\end{aligned}
$$


are chiral and antichiral respectively: $\bar{D} Y=0 ; D \bar{Y}=0$. For the special case when $D \delta \Lambda=\delta \lambda$ and $\bar{D} \delta \bar{\Lambda}=-\delta \bar{\lambda}$, where $\delta \lambda$ and $\delta \bar{\lambda}$ are constant Grassmann odd superconformal transformation parameters, the $Y$ and $\bar{Y}$ terms can be included as $F$ and $\bar{F}$ terms in $\delta \mathcal{L}$. Since the purely chiral and antichiral terms $F+\bar{F}$ do not contribute to the variation of the action, they may be absorbed into the definition of the Noether charge $\mathcal{Q}$ leading to an improved Noether charge $\mathcal{Q}_{I}$ given by

$$
\mathcal{Q}_{I} \equiv \mathcal{Q}+\frac{1}{2}(\bar{F}-F)
$$

with $\mathcal{Q}$ defined in equation (3.4). Finally, introducing the combination

$$
\Delta \mathcal{L} \equiv \delta \mathcal{L}-(F+\bar{F})
$$

the improved Noether charge obeys the identities

$$
\begin{aligned}
& D \mathcal{Q}_{I}=\delta \bar{\phi} \frac{\delta \Gamma_{0}}{\delta \bar{\phi}}+\frac{1}{2} D \Delta \mathcal{L} \\
& \bar{D} \mathcal{Q}_{I}=-\delta \phi \frac{\delta \Gamma_{0}}{\delta \phi}-\frac{1}{2} \bar{D} \Delta \mathcal{L}
\end{aligned}
$$

and the conservation relation

$$
-2 i \frac{\partial}{\partial t} \mathcal{Q}_{I}=D\left(\delta \phi \frac{\delta \Gamma_{0}}{\delta \phi}\right)-\bar{D}\left(\delta \bar{\phi} \frac{\delta \Gamma_{0}}{\delta \bar{\phi}}\right)+\frac{1}{2}[D, \bar{D}] \Delta \mathcal{L} .
$$

Integrating over the time yields the quantum action principle or Ward identity

$$
\delta \Gamma=\left[i \int d V \delta \mathcal{L}\right] \Gamma
$$

Thus, if the classical action is invariant, $\int d V \delta \mathcal{L}=0$, so is the effective action $\delta \Gamma=0$.

The improved superconformal charges take the form

$$
\begin{aligned}
\mathcal{Q}_{I}= & \frac{1}{2}\left\{\frac{i}{2}(\delta \Lambda+\delta \bar{\Lambda})\left(\phi K_{\phi}+\bar{\phi} K_{\bar{\phi}}\right)+\frac{i}{2}(\delta \Lambda-\delta \bar{\Lambda})\left(\phi K_{\phi}-\bar{\phi} K_{\bar{\phi}}\right)\right. \\
& +i \delta B K_{\phi \bar{\phi}} D \phi \bar{D} \bar{\phi}+4 i \delta B g \dot{\phi} \overline{\bar{\phi}}-i(\delta \Lambda+\delta \bar{\Lambda}+D X-\bar{D} \bar{X}) g D \phi \bar{D} \bar{\phi} \\
& -\frac{i}{2}(\delta \Lambda+\delta \bar{\Lambda})\left(\phi g_{\phi}+\bar{\phi} g_{\bar{\phi}}\right) D \phi \bar{D} \bar{\phi}-\frac{i}{2}(\delta \Lambda-\delta \bar{\Lambda})\left(\phi g_{\phi}-\bar{\phi} g_{\bar{\phi}}\right) D \phi \bar{D} \bar{\phi} \\
& +2(\delta \Lambda+\delta \bar{\Lambda}) g(\phi \dot{\bar{\phi}}-\dot{\phi} \bar{\phi})+2(\delta \Lambda-\delta \bar{\Lambda}) g(\phi \dot{\bar{\phi}}+\dot{\phi} \bar{\phi}) \\
& -2 i D \delta \Lambda g \phi \bar{D} \bar{\phi}+2 i \bar{D} \delta \bar{\Lambda} g D \phi \bar{\phi}-i D \delta \Lambda g_{\bar{\phi}} \bar{\phi} \phi \bar{D} \bar{\phi}+i \bar{D} \delta \Lambda g_{\phi} \phi D \phi \bar{\phi} \\
& +2(\delta \bar{\Lambda}-\delta \dot{\Lambda}) g \phi \bar{\phi}+4 \delta X g D \phi \dot{\bar{\phi}}+4 \delta \bar{X} g \dot{\phi} \bar{D} \bar{\phi}\}
\end{aligned}
$$

Substituting the explicit expressions for the superspace dependent transformation parameters and isolating the coefficients of the various c-number and Grassmann odd parameters, 
the superconformal charges (defined with the transformation parameters removed from their definitions) and (non-) conservation laws can be extracted. Below we compile the various superspace dependent (improved) charges whose $\theta, \bar{\theta}$ independent components generate the associated superconformal symmetries. In addition, we give the SUSY covariant derivatives of these superspace charges, their (non-) conservation laws and the form of the explicit breaking terms.

Throughout this section as well as in section 4, we adapt the notation that $H, Q, \bar{Q}, K, S, \bar{S}, R$ and $J$ represent an entire function of superspace whose $\theta, \bar{\theta}$ independent component is the actual superconformal and $U_{J}(1)$ generator. Elsewhere in the paper, these same symbols are used to represent just the actual generators.

1. Time Translations: Parameter $\delta \epsilon$

$$
\begin{aligned}
\mathcal{Q}_{I}^{H}=H & \equiv 4 g \dot{\phi} \dot{\bar{\phi}}+K_{\phi \bar{\phi}} D \phi \bar{D} \bar{\phi} \\
D H & =2 i \dot{\bar{\phi}} \frac{\delta \Gamma_{0}}{\delta \bar{\phi}} \\
\bar{D} H & =-2 i \dot{\phi} \frac{\delta \Gamma_{0}}{\delta \phi} \\
\frac{\partial}{\partial t} H & =-D\left(\dot{\phi} \frac{\delta \Gamma_{0}}{\delta \phi}\right)+\bar{D}\left(\dot{\bar{\phi}} \frac{\delta \Gamma_{0}}{\delta \bar{\phi}}\right) \\
\frac{1}{2}[D, \bar{D}] H & =-i\left(D\left(\dot{\phi} \frac{\delta \Gamma_{0}}{\delta \phi}\right)+\bar{D}\left(\dot{\bar{\phi}} \frac{\delta \Gamma_{0}}{\delta \bar{\phi}}\right)\right) \\
\Delta^{H} \mathcal{L} & =0 .
\end{aligned}
$$

2. Supersymmetry: Parameters $\delta \eta$ and $\delta \bar{\eta}$

$$
\begin{aligned}
\mathcal{Q}_{I}^{Q} & =Q-2 i \bar{\theta} H \\
\mathcal{Q}_{I}^{\bar{Q}} & =\bar{Q}+2 i \theta H \\
\frac{\partial}{\partial t} \mathcal{Q}_{I}^{Q} & =-\left(D\left(\delta^{Q} \phi \frac{\delta \Gamma_{0}}{\delta \phi}\right)-\bar{D}\left(\delta^{Q} \bar{\phi} \frac{\delta \Gamma_{0}}{\delta \bar{\phi}}\right)\right) \\
\frac{\partial}{\partial t} \mathcal{Q}_{I}^{\bar{Q}} & =-\left(D\left(\delta^{\bar{Q}} \phi \frac{\delta \Gamma_{0}}{\delta \phi}\right)-\bar{D}\left(\delta^{\bar{Q}} \bar{\phi} \frac{\delta \Gamma_{0}}{\delta \bar{\phi}}\right)\right) \\
\Delta^{\mathcal{Q}_{I}^{Q}} \mathcal{L}=0 & =\Delta^{\mathcal{Q}_{I}^{\bar{Q}}} \mathcal{L},
\end{aligned}
$$

where

$$
Q \equiv-4 g D \phi \dot{\bar{\phi}}
$$




$$
\begin{aligned}
\bar{Q} \equiv & -4 g \dot{\phi} \bar{D} \bar{\phi} \\
D Q= & =\bar{D} \bar{Q} \\
\bar{D} Q= & -2 i H-2 i D \phi \frac{\delta \Gamma_{0}}{\delta \phi} \\
D \bar{Q}= & -2 i H+2 i \bar{D} \bar{\phi} \frac{\delta \Gamma_{0}}{\delta \bar{\phi}} \\
\frac{\partial}{\partial t} Q= & -\left\{D\left(\delta^{Q} \phi \frac{\delta \Gamma_{0}}{\delta \phi}\right)-\bar{D}\left(\delta^{Q} \bar{\phi} \frac{\delta \Gamma_{0}}{\delta \bar{\phi}}\right)\right\} \\
& -2 i \bar{\theta}\left\{D\left(\dot{\phi} \frac{\delta \Gamma_{0}}{\delta \phi}\right)-\bar{D}\left(\dot{\bar{\phi}} \frac{\delta \Gamma_{0}}{\delta \bar{\phi}}\right)\right\} \\
\frac{\partial}{\partial t} \bar{Q}= & -\left\{D\left(\delta^{\bar{Q}} \phi \frac{\delta \Gamma_{0}}{\delta \phi}\right]-\bar{D}\left[\delta^{\bar{Q}} \bar{\phi} \frac{\delta \Gamma_{0}}{\delta \bar{\phi}}\right)\right\} \\
& +2 i \theta\left\{D\left(\dot{\phi} \frac{\delta \Gamma_{0}}{\delta \phi}\right)-\bar{D}\left(\dot{\bar{\phi}} \frac{\delta \Gamma_{0}}{\delta \bar{\phi}}\right)\right\} .
\end{aligned}
$$

(Note that a in slight variation from the usual notational convention $Q$ is antichiral, $(D Q=0)$, while $\bar{Q}$ is chiral, $(\bar{D} \bar{Q}=0)$.)

3. $U_{J}(1)$ Transformations: Parameter $\delta \alpha$

$$
\begin{aligned}
\mathcal{Q}_{I}^{U(1)} & =J \equiv-2 i g D \phi \bar{D} \bar{\phi}+4 g(\phi \dot{\bar{\phi}}-\dot{\phi} \bar{\phi})-i\left(\phi g_{\phi}+\bar{\phi} g_{\bar{\phi}}\right) D \phi \bar{D} \bar{\phi}+i\left(\phi K_{\phi}+\bar{\phi} K_{\bar{\phi}}\right) \\
D J & =-2 i \bar{\phi} \frac{\delta \Gamma_{0}}{\delta \bar{\phi}}+\frac{1}{2} D \Delta^{U(1)} \mathcal{L} \\
\bar{D} J & =-2 i \phi \frac{\delta \Gamma_{0}}{\delta \phi}-\frac{1}{2} \bar{D} \Delta^{U(1)} \mathcal{L} \\
\frac{\partial}{\partial t} J & =-D\left(\phi \frac{\delta \Gamma_{0}}{\delta \phi}\right)-\bar{D}\left(\bar{\phi} \frac{\delta \Gamma_{0}}{\delta \bar{\phi}}\right)+\frac{i}{4}[D, \bar{D}] \Delta^{U(1)} \mathcal{L} \\
\Delta^{U(1)} \mathcal{L} & =2 i\left\{\left(\phi K_{\phi}-\bar{\phi} K_{\bar{\phi}}\right)+\left(\phi g_{\phi}-\bar{\phi} g_{\bar{\phi}}\right) D \phi \bar{D} \bar{\phi}\right\}
\end{aligned}
$$

4. $R$ Transformations: Parameter $n_{\phi} \delta \alpha+\delta \rho$

$$
\begin{aligned}
\mathcal{Q}_{I}^{R} & =\frac{1}{2}(R-\theta Q-\bar{\theta} \bar{Q}+2 i \theta \bar{\theta} H) \\
2 i \frac{\partial}{\partial t} \mathcal{Q}_{I}^{R} & =-D\left(\delta^{R} \phi \frac{\delta \Gamma_{0}}{\delta \phi}\right)+\bar{D}\left(\delta^{R} \bar{\phi} \frac{\delta \Gamma_{0}}{\delta \bar{\phi}}\right)-\frac{1}{2}[D, \bar{D}] \Delta^{R} \mathcal{L} \\
\Delta^{R} \mathcal{L} & =\frac{n_{\phi}}{2} \Delta^{U(1)} \mathcal{L}=i n_{\phi}\left\{\left(\phi K_{\phi}-\bar{\phi} K_{\bar{\phi}}\right)+\left(\phi g_{\phi}-\bar{\phi} g_{\bar{\phi}}\right) D \phi \bar{D} \bar{\phi}\right\}
\end{aligned}
$$

where

$$
\begin{aligned}
R & \equiv n_{\phi} J+R_{0} \\
& =-2 i\left(1+n_{\phi}\right) g D \phi \bar{D} \bar{\phi}+4 n_{\phi} g(\phi \dot{\bar{\phi}}-\dot{\phi} \bar{\phi})
\end{aligned}
$$




$$
-i n_{\phi}\left(\phi g_{\phi}+\bar{\phi} g_{\bar{\phi}}\right) D \phi \bar{D} \bar{\phi}+i n_{\phi}\left(\phi K_{\phi}+\bar{\phi} K_{\bar{\phi}}\right)
$$

For $n_{\phi}=0$, the conserved supercoordinate charge $R_{0}$ is obtained as

$$
\begin{aligned}
R_{0} & \equiv-2 i g D \phi \bar{D} \bar{\phi} \\
D R_{0} & =Q \\
\bar{D} R_{0} & =-\bar{Q} \\
2 i \frac{\partial}{\partial t} R_{0} & =\{D, \bar{D}\} R_{0}=-2 i\left(D \phi \frac{\delta \Gamma_{0}}{\delta \phi}+\bar{D} \bar{\phi} \frac{\delta \Gamma_{0}}{\delta \bar{\phi}}\right) \\
\frac{1}{2}[D, \bar{D}] R_{0} & =2 i H+i\left(D \phi \frac{\delta \Gamma_{0}}{\delta \phi}-\bar{D} \bar{\phi} \frac{\delta \Gamma_{0}}{\delta \bar{\phi}}\right) .
\end{aligned}
$$

It should be pointed out that $R_{0}$ may also be obtained by directly applying Noether's theorem to the case that the supercoordinate variations are covariant derivatives (in which case that the transformations do not preserve the original chirality of the supercoordinates). Considering the infinitesimal transformations $\delta \phi=\delta \zeta D \phi$ and $\delta \bar{\phi}=\delta \bar{\zeta} \bar{D} \bar{\phi}$, where $\delta \zeta$ and $\delta \bar{\zeta}$ are independent complex anticommuting parameters, equation (3.4) yields the associated Noether charge

$$
\mathcal{Q}=(\delta \zeta D-\delta \bar{\zeta} \bar{D})\left(g D \phi \bar{D} \bar{\phi}+\frac{1}{2} K\right)
$$

while equation (3.1) gives the variation of $\mathcal{L}$ as

$$
\delta \mathcal{L}=(\delta \zeta D+\delta \bar{\zeta} \bar{D}) K
$$

Absorbing $D K$ and $\bar{D} K$ into $\mathcal{Q}$ defines the improved charge $\mathcal{Q}_{I}$ as

$$
\begin{aligned}
\mathcal{Q}_{I} & =\mathcal{Q}-\frac{1}{2}(\delta \zeta D K-\delta \bar{\zeta} \bar{D} K) \\
& =\frac{i}{2}(\delta \zeta D-\delta \bar{\zeta} \bar{D}) R_{0}=\frac{i}{2}(\delta \zeta Q+\delta \bar{\zeta} \bar{Q}) .
\end{aligned}
$$

The derivative equations are easily obtained as

$$
\begin{aligned}
D \mathcal{Q}_{I} & =\frac{i}{2} \delta \bar{\zeta} D \bar{D} R_{0}=-\frac{i}{2} \delta \bar{\zeta} D \bar{Q}=-\delta \bar{\zeta}\left(H-\bar{D} \bar{\phi} \frac{\delta \Gamma_{0}}{\delta \bar{\phi}}\right) \\
\bar{D} \mathcal{Q}_{I} & =-\frac{i}{2} \delta \zeta \bar{D} D R_{0}=-\frac{i}{2} \delta \zeta \bar{D} Q=-\delta \zeta\left(H+D \phi \frac{\delta \Gamma_{0}}{\delta \phi}\right)
\end{aligned}
$$

leading to the time derivative 


$$
\frac{\partial}{\partial t} R_{0}=-\left(D \phi \frac{\delta \Gamma_{0}}{\delta \phi}+\bar{D} \bar{\phi} \frac{\delta \Gamma_{0}}{\delta \bar{\phi}}\right)
$$

In a similar manner, the chiral and antichiral supercoordinate counting densities can be found by considering the number operator variations of the supercoordinates (global Weyl scaling)

$$
\begin{array}{rlrl}
\delta^{N} \phi & =\phi & & \delta^{\bar{N}} \phi=0 \\
\delta^{N} \bar{\phi}=0 & \delta^{\bar{N}} \bar{\phi}=\bar{\phi} .
\end{array}
$$

Noether's theorem yields the counting charges

$$
\begin{aligned}
\mathcal{Q}^{N} & =\frac{1}{2}\left(-g D \phi \bar{D} \bar{\phi}-\phi g_{\phi} D \phi \bar{D} \bar{\phi}+\phi K_{\phi}-4 i g \phi \dot{\bar{\phi}}\right) \\
\mathcal{Q}^{\bar{N}} & =\frac{1}{2}\left(+g D \phi \bar{D} \bar{\phi}+\bar{\phi} g_{\bar{\phi}} D \phi \bar{D} \bar{\phi}-\bar{\phi} K_{\bar{\phi}}-4 i g \dot{\phi} \bar{\phi}\right)
\end{aligned}
$$

and SUSY covariant derivatives

$$
\begin{aligned}
D \mathcal{Q}^{N} & =\frac{1}{2} D \delta^{N} \mathcal{L} \\
\bar{D} \mathcal{Q}^{N} & =-\phi \frac{\delta \Gamma_{0}}{\delta \phi}-\frac{1}{2} \bar{D} \delta^{N} \mathcal{L} \\
D \mathcal{Q}^{\bar{N}} & =\bar{\phi} \frac{\delta \Gamma_{0}}{\delta \bar{\phi}}+\frac{1}{2} D \delta^{\bar{N}} \mathcal{L} \\
\bar{D} \mathcal{Q}^{\bar{N}} & =-\frac{1}{2} \bar{D} \delta^{\bar{N}} \mathcal{L},
\end{aligned}
$$

where

$$
\begin{aligned}
\delta^{N} \mathcal{L} & =\frac{i}{2} R_{0}+\phi K_{\phi}+\phi g_{\phi} D \phi \bar{D} \bar{\phi} \\
& =\left(1+\phi(\ln g)_{\phi}\right) g D \phi \bar{D} \bar{\phi}+\phi(\ln K)_{\phi} K \\
\delta^{\bar{N}} \mathcal{L} & =\frac{i}{2} R_{0}+\bar{\phi} K_{\bar{\phi}}+\bar{\phi} g_{\bar{\phi}} D \phi \bar{D} \bar{\phi} \\
& =\left(1+\bar{\phi}(\ln g)_{\bar{\phi}}\right) g D \phi \bar{D} \bar{\phi}+\bar{\phi}(\ln K)_{\bar{\phi}} K .
\end{aligned}
$$

Thus the (non-) conservation equations for the counting density are given by

$$
\begin{aligned}
\{D, \bar{D}\} \mathcal{Q}^{N} & =2 i \frac{\partial}{\partial t} \mathcal{Q}^{N} \\
& =-D\left(\phi \frac{\delta \Gamma_{0}}{\delta \phi}\right)-\frac{1}{2}[D, \bar{D}] \delta^{N} \mathcal{L} \\
\{D, \bar{D}\} \mathcal{Q}^{\bar{N}} & =2 i \frac{\partial}{\partial t} \mathcal{Q}^{\bar{N}}
\end{aligned}
$$




$$
=\bar{D}\left(\bar{\phi} \frac{\delta \Gamma_{0}}{\delta \bar{\phi}}\right)-\frac{1}{2}[D, \bar{D}] \delta^{\bar{N}} \mathcal{L}
$$

Combining these chiral and antichiral supercoordinate counting equations, one secures the total supercoordinate number operator (global Weyl scaling) conservation identity:

$$
\begin{aligned}
\frac{\partial}{\partial t} U & \equiv 2 i \frac{\partial}{\partial t}\left(\mathcal{Q}^{N}+\mathcal{Q}^{\bar{N}}\right) \\
& =-D\left(\phi \frac{\delta \Gamma_{0}}{\delta \phi}\right)+\bar{D}\left(\bar{\phi} \frac{\delta \Gamma_{0}}{\delta \bar{\phi}}\right)+\frac{1}{2}[D, \bar{D}]\left(\delta^{N} \mathcal{L}+\delta^{\bar{N}} \mathcal{L}\right)
\end{aligned}
$$

This may be alternatively expressed by considering the chiral and antichiral counting variations of the Lagrangian directly. Introducing the chiral and antichiral combinations

$$
\begin{array}{ll}
T=-i g \phi \bar{D} \bar{\phi} ; & (\bar{D} T=0) \\
\bar{T}=-i g D \phi \bar{\phi} \quad ; \quad(D \bar{T}=0)
\end{array}
$$

with the derivative identities

$$
\begin{aligned}
& D T=-i \delta^{N} \mathcal{L}-i \frac{1}{\bar{D}}\left(\phi \frac{\delta \Gamma_{0}}{\delta \phi}\right) \\
& \bar{D} \bar{T}=i \delta^{\bar{N}} \mathcal{L}+i \frac{1}{D}\left(\bar{\phi} \frac{\delta \Gamma_{0}}{\delta \bar{\phi}}\right)
\end{aligned}
$$

the total number density can be written as

$$
\begin{aligned}
U & =4 g \frac{\partial}{\partial t}(\phi \bar{\phi})+i\left(\phi K_{\phi}-\bar{\phi} K_{\bar{\phi}}\right)-i\left(\phi g_{\phi}-\bar{\phi} g_{\bar{\phi}}\right) D \phi \bar{D} \bar{\phi} \\
& =D T+\bar{D} \bar{T}+i\left\{\frac{1}{D}\left(\bar{\phi} \frac{\delta \Gamma_{0}}{\delta \bar{\phi}}\right)-\frac{1}{\bar{D}}\left(\phi \frac{\delta \Gamma_{0}}{\delta \phi}\right)\right\}
\end{aligned}
$$

The conservation equations take the form

$$
\begin{aligned}
& D U=2 i \bar{\phi} \frac{\delta \Gamma_{0}}{\delta \bar{\phi}}+i D \delta^{U} \mathcal{L} \\
& \bar{D} U=-2 i \phi \frac{\delta \Gamma_{0}}{\delta \phi}-i \bar{D} \delta^{U} \mathcal{L}
\end{aligned}
$$

from which the time derivative is obtained as usual reproducing equation (3.53)

$$
-\frac{\partial}{\partial t} U=D\left(\phi \frac{\delta \Gamma_{0}}{\delta \phi}\right)-\bar{D}\left(\bar{\phi} \frac{\delta \Gamma_{0}}{\delta \bar{\phi}}\right)+\frac{1}{2}[D, \bar{D}] \delta^{U} \mathcal{L}
$$


where

$$
\delta^{U} \mathcal{L}=2 i\left(\delta^{N} \mathcal{L}+\delta^{\bar{N}} \mathcal{L}\right)
$$

So defined, these are the counting operators which enter into the scaling and conformal SUSY charges.

5. Dilatations: Parameter $\delta \beta$

$$
\begin{aligned}
\mathcal{Q}_{I}^{D} & =-\frac{i}{2}\left(-t H+\frac{1}{2} U+\frac{1}{2} \theta Q-\frac{1}{2} \bar{\theta} \bar{Q}\right) \\
2 i \frac{\partial}{\partial t} \mathcal{Q}_{I}^{D} & =D\left(\delta^{D} \phi \frac{\delta \Gamma_{0}}{\delta \phi}\right)-\bar{D}\left(\delta^{D} \bar{\phi} \frac{\delta \Gamma_{0}}{\delta \bar{\phi}}\right)-\frac{1}{2}[D, \bar{D}] \Delta^{D} \mathcal{L} \\
\Delta^{D} \mathcal{L} & =-\frac{i}{4}\left(\delta^{U} \mathcal{L}+2 R_{0}\right) \\
& =\frac{1}{2}\left(\left(\phi K_{\phi}+\bar{\phi} K_{\bar{\phi}}\right)+\left(\phi g_{\phi}+\bar{\phi} g_{\bar{\phi}}\right) D \phi \bar{D} \bar{\phi}\right) .
\end{aligned}
$$

6. Superconformal Symmetry: Parameters $\delta \lambda$ and $\delta \bar{\lambda}$

$$
\begin{aligned}
\mathcal{Q}_{I}^{S} & =-\frac{i}{2} t \bar{Q}+T-i \bar{D}(g \phi \bar{\phi})-\theta\left(2 i \mathcal{Q}_{I}^{D}+\frac{1}{2} R\right) \\
& =-\frac{i}{2} t \bar{Q}+2 T-i Y-\theta\left(2 i \mathcal{Q}_{I}^{D}+\frac{1}{2} R\right) \\
\mathcal{Q}_{I}^{\bar{S}} & =-\frac{i}{2} t Q+\bar{T}-i D(g \phi \bar{\phi})+\bar{\theta}\left(2 i \mathcal{Q}_{I}^{D}-\frac{1}{2} R\right) \\
& =-\frac{i}{2} t Q+2 \bar{T}-i \bar{Y}+\bar{\theta}\left(2 i \mathcal{Q}_{I}^{D}-\frac{1}{2} R\right) \\
2 i \frac{\partial}{\partial t} \mathcal{Q}_{I}^{S} & =D\left(\delta^{S} \phi \frac{\delta \Gamma_{0}}{\delta \phi}\right)-\bar{D}\left(\delta^{S} \bar{\phi} \frac{\delta \Gamma_{0}}{\delta \bar{\phi}}\right)-\frac{1}{2}[D, \bar{D}] \Delta^{S} \mathcal{L} \\
2 i \frac{\partial}{\partial t} \mathcal{Q}_{I}^{\bar{S}} & =D\left(\delta^{\bar{S}} \phi \frac{\delta \Gamma_{0}}{\delta \phi}\right)-\bar{D}\left(\delta^{\bar{S}} \bar{\phi} \frac{\delta \Gamma_{0}}{\delta \bar{\phi}}\right)-\frac{1}{2}[D, \bar{D}] \Delta^{\bar{S}} \mathcal{L} \\
\Delta^{S} \mathcal{L} & =-2 i \bar{\phi} g_{\bar{\phi}} \phi \bar{D} \bar{\phi}-\theta\left(2 i \delta^{N} \mathcal{L}+R_{0}\right) \\
& =-2 i Y-\theta\left(2 i \delta^{N} \mathcal{L}+R_{0}\right) \\
\Delta^{\bar{S}} \mathcal{L} & =2 i \phi g_{\phi} D \phi \bar{\phi}+\bar{\theta}\left(2 i \delta^{\bar{N}} \mathcal{L}+R_{0}\right) \\
& =2 i \bar{Y}+\bar{\theta}\left(2 i \delta^{\bar{N}} \mathcal{L}+R_{0}\right) .
\end{aligned}
$$

As alluded to in the discussion following equations (3.27) and (3.28), as a consequence of their chiral and antichiral nature, the superconformal symmetry breaking terms due to $Y$ and $\bar{Y}$ can be absorbed into the charges to form new, improved charges. Defining these as $\mathcal{Q}_{N I}^{S}=\mathcal{Q}_{I}^{S}+i Y$ and $\mathcal{Q}_{N I}^{\bar{S}}=\mathcal{Q}_{I}^{\bar{S}}+i \bar{Y}$, the associated conservation equations are given by 


$$
\begin{aligned}
& 2 i \frac{\partial}{\partial t} \mathcal{Q}_{N I}^{S}=D\left(\delta^{S} \phi \frac{\delta \Gamma_{0}}{\delta \phi}\right)-\bar{D}\left(\delta^{S} \bar{\phi} \frac{\delta \Gamma_{0}}{\delta \bar{\phi}}\right)-\frac{1}{2}[D, \bar{D}] \delta^{S_{N I}} \mathcal{L} \\
& 2 i \frac{\partial}{\partial t} \mathcal{Q}_{N I}^{\bar{S}}=D\left(\delta^{\bar{S}} \phi \frac{\delta \Gamma_{0}}{\delta \phi}\right)-\bar{D}\left(\delta^{\bar{S}} \bar{\phi} \frac{\delta \Gamma_{0}}{\delta \bar{\phi}}\right)-\frac{1}{2}[D, \bar{D}] \delta^{\bar{S} N I} \mathcal{L}
\end{aligned}
$$

where the breaking terms are now just

$$
\begin{aligned}
& \delta^{S N I} \mathcal{L}=-\theta\left(2 i \delta^{N} \mathcal{L}+R_{0}\right) \\
& \delta^{\bar{S} N I} \mathcal{L}=\bar{\theta}\left(2 i \delta^{\bar{N}} \mathcal{L}+R_{0}\right)
\end{aligned}
$$

7. Conformal Symmetry: Parameter $\delta \gamma$

$$
\begin{aligned}
\mathcal{Q}_{I}^{K}= & \frac{i}{2}\left(t U-t^{2} H\right)-2 i g \phi \bar{\phi}-\theta \mathcal{Q}_{I}^{\bar{S}}+\bar{\theta} \mathcal{Q}_{I}^{S}-\frac{1}{2} \theta \bar{\theta} R \\
= & \frac{i}{2}\left\{\begin{array}{c}
t U-t^{2} H-4 g \phi \bar{\phi}-i \theta \bar{\theta} R \\
+\theta\left(t Q+4 g D \phi \bar{\phi}+2 \phi g_{\phi} D \phi \bar{\phi}\right)
\end{array}\right. \\
& \left.-\bar{\theta}\left(t \bar{Q}+4 g \phi \bar{D} \bar{\phi}+2 \bar{\phi} g_{\bar{\phi}} \phi \bar{D} \bar{\phi}\right)\right\} \\
2 i \frac{\partial}{\partial t} \mathcal{Q}_{I}^{K}= & -D\left(\delta^{K} \phi \frac{\delta \Gamma_{0}}{\delta \phi}\right)+\bar{D}\left(\delta^{K} \bar{\phi} \frac{\delta \Gamma_{0}}{\delta \bar{\phi}}\right)-\frac{1}{2}[D, \bar{D}] \delta^{K} \mathcal{L} \\
\delta^{K} \mathcal{L}= & -2 t \delta^{D} \mathcal{L}-\theta \delta^{\bar{S}} \mathcal{L}+\bar{\theta} \delta^{S} \mathcal{L}-\frac{1}{2} \theta \bar{\theta} \delta^{U(1)} \mathcal{L} \\
= & -2 t \delta^{D} \mathcal{L}-2 i \theta \phi g_{\phi} D \phi \bar{\phi}-2 i \bar{\theta} \bar{\phi} g_{\bar{\phi}} \phi \bar{D} \bar{\phi}+\frac{1}{2} \theta \bar{\theta} \delta^{U(1)} \mathcal{L} \\
= & -2 t \delta^{D} \mathcal{L}-2 i \theta \bar{Y}-2 i \bar{\theta} Y+\frac{1}{2} \theta \bar{\theta} \delta^{U(1)} \mathcal{L} .
\end{aligned}
$$

A new, improved conformal charge can also be defined by absorbing part of the $Y$ and $\bar{Y}$ breaking terms into the charge. These terms in turn will become part of the new, improved superconformal charges replacing the improved ones in the conformal charge as

$$
\mathcal{Q}_{N I}^{K}=\frac{i}{2}\left(t U-t^{2} H\right)-2 i g \phi \bar{\phi}-\theta \mathcal{Q}_{N I}^{\bar{S}}+\bar{\theta} \mathcal{Q}_{N I}^{S}-\frac{1}{2} \theta \bar{\theta} R
$$

However there still remains $Y$ and $\bar{Y}$ dependent terms in the conformal symmetry (non-)conservation equation:

$$
\begin{aligned}
2 i \frac{\partial}{\partial t} \mathcal{Q}_{N I}^{K} & =-D\left(\delta^{K} \phi \frac{\delta \Gamma_{0}}{\delta \phi}\right)+\bar{D}\left(\delta^{K} \bar{\phi} \frac{\delta \Gamma_{0}}{\delta \bar{\phi}}\right)-\frac{1}{2}[D, \bar{D}] \Delta_{N I}^{K} \mathcal{L}-2 i D Y-2 i \bar{D} \bar{Y} \\
\Delta_{N I}^{K} \mathcal{L} & =-2 t \Delta^{D} \mathcal{L}+\frac{1}{2} \theta \bar{\theta} \Delta^{U(1)} \mathcal{L} .
\end{aligned}
$$


By appealing to the action principle, the (local) variations of the effective action, and, hence, the Green's functions, are given by the above equations. For general variations $\delta \phi$ and $\delta \bar{\phi}$, the action principle yields

$$
\begin{aligned}
& i[\delta \phi(t, \theta, \bar{\theta})\left.\frac{\delta \Gamma_{0}}{\delta \phi(t, \theta, \bar{\theta})}\right] \Gamma=\delta \phi(t, \theta, \bar{\theta}) \frac{\delta}{\delta \phi(t, \theta, \bar{\theta})} \Gamma \\
& i\left[\delta \bar{\phi}(t, \theta, \bar{\theta}) \frac{\delta \Gamma_{0}}{\delta \bar{\phi}(t, \theta, \bar{\theta})}\right] \Gamma=\delta \bar{\phi}(t, \theta, \bar{\theta}) \frac{\delta}{\delta \bar{\phi}(t, \theta, \bar{\theta})} \Gamma .
\end{aligned}
$$

Introducing the Ward identity functional differential operators for each of the variations of the superconformal and $U(1)$ transformations, $\mathcal{Q} \in\{H, Q, \bar{Q}, R, J, D, K, S, \bar{S}\}$,

$$
\delta^{\mathcal{Q}} \equiv \int d S \delta^{\mathcal{Q}} \phi \frac{\delta}{\delta \phi}+\int d \bar{S} \delta^{\mathcal{Q}} \bar{\phi} \frac{\delta}{\delta \bar{\phi}},
$$

and the local chiral and antichiral Ward identity operators

$$
\begin{aligned}
w^{\mathcal{Q}} & \equiv D\left(\delta^{\mathcal{Q}} \phi \frac{\delta}{\delta \phi}\right) \\
\bar{w}^{\mathcal{Q}} & \equiv \bar{D}\left(\delta^{\mathcal{Q}} \bar{\phi} \frac{\delta}{\delta \bar{\phi}}\right),
\end{aligned}
$$

Noether's theorem has the general form

$$
w^{\mathcal{Q}} \Gamma-\bar{w}^{\mathcal{Q}} \Gamma=2 \frac{\partial}{\partial t} \mathcal{Q}_{I}-\frac{i}{2}[D, \bar{D}] \Delta^{\mathcal{Q}} \mathcal{L} .
$$

Integrating over the time, and discarding total time differentials secures the quantum action principle

$$
\delta^{\mathcal{Q}} \Gamma=\left[\int d V \Delta^{\mathcal{Q}} \mathcal{L}\right] \Gamma
$$

where once again

$$
\delta^{\mathcal{Q}}=\int d t\left(w^{\mathcal{Q}}-\bar{w}^{\mathcal{Q}}\right)=\int d S \delta^{\mathcal{Q}} \phi \frac{\delta}{\delta \phi}+\int d \bar{S} \delta^{\mathcal{Q}} \bar{\phi} \frac{\delta}{\delta \bar{\phi}} .
$$

The variations of the Green's functions under superconformal and $U(1)$ transformations are obtained as Ward identity functional differential equations for the effective action. Alternatively, the operator inserted Green's functions determine the matrix elements of the operators through the reduction formalism. The local Ward identity functional differential operator terms vanish asymptotically, hence Noether's theorem can be viewed as an operator relation between the time derivative of the charge and the associated variation of the Lagrangian

$$
2 i \frac{\partial}{\partial t} \mathcal{Q}_{I}=-\frac{1}{2}[D, \bar{D}] \Delta^{\mathcal{Q}} \mathcal{L}
$$




\section{SUPERCHARGES}

As discussed in the previous section, it is the $\theta$ and $\bar{\theta}$ independent components of the superspace dependent Noether charges which serve as the superconformal and $U_{J}(1)$ generators. Although these Noether charges are functions of superspace, they do not all transform as supercoordinates. While some are supercoordinates, others involve the product of explicit factors of superspace, $(t, \theta, \bar{\theta})$, multiplying supercoordinates. Just as the Noether charges do not form supercoordinates neither do the respective variations of the Lagrangian. Using the superconformal algebra, however, the charges can be assembled into multiplet structures whose components transform into each other under a restricted set of SUSY transformations which are parametrized by the anticommuting superspace coordinates $\theta, \bar{\theta}$ themselves rather than by arbitrary anticommuting parameters. Such multiplet structures are referred to as quasi-supercoordinates [8]. The restricted supersymmetry relating the Noether charges can also be used to connect the various symmetry breaking terms. These quasi-supercoordinates of charges (called supercharges) are constructed in Appendix B. The quasi-supercoordinates of charges are all constructed from the Hamiltonian and SUSY charge supercoordinates \& and the supercoordinate charges associated with the chiral and antichiral global Weyl scaling of the supercoordinates (that is the chiral and antichiral number operators). The $U_{J}(1)$ symmetry Noether charge forms its own supercoordinate. Due to the quasi-supersymmetry covariance, the variations of the action under the various symmetry transformations are also related. In fact all superconformal variations of the action are obtained from combinations of the $U_{J}(1)$ symmetry variation of the Lagrangian and the conformal SUSY variations of the Lagrangian.

Since the Noether construction is a SUSY covariant proceedure, as long as the variation of the supercoordinates under consideration is SUSY covariant, the resulting Noether charge will be a supercoordinate. Indeed, the $U_{J}(1)$ transformation of the supercoordinates is a covariant transformation in that it commutes with the SUSY variations of the coordinates. Thus the Noether construction of the $U_{J}(1)$ charge results in a supercoordinate. Once again, it is the $\theta, \bar{\theta}$ independent component which is the actual generator, while the time derivative

\footnotetext{
${ }^{1}$ Note that just as is the case in supersymmetric field theory, the Hamiltonian and SUSY charges can be reconstructed from the $R$ symmetry charge [9].
} 
of the higher components simply integrate to zero. The supercharge given in equation (3.39)

$$
J=-2 i g D \phi \bar{D} \bar{\phi}+4 g(\phi \dot{\bar{\phi}}-\dot{\phi} \bar{\phi})-i\left(\phi g_{\phi}+\bar{\phi} g_{\bar{\phi}}\right) D \phi \bar{D} \bar{\phi}+i\left(\phi K_{\phi}+\bar{\phi} K_{\bar{\phi}}\right)
$$

can be expanded in components as

$$
J=J_{00}+\theta \sqrt{2} J_{10}+\bar{\theta} \sqrt{2} J_{01}+\theta \bar{\theta} J_{11}
$$

where the $\theta, \bar{\theta}$ independent component is the $U_{J}(1)$ symmetry generator

$$
J_{00}=j=-4 i g \xi \bar{\xi}-4 g(\dot{z} \bar{z}-z \dot{\bar{z}})+i\left(z K_{z}+\bar{z} K_{\bar{z}}\right)-2 i\left(z g_{z}+\bar{z} g_{\bar{z}}\right) \xi \bar{\xi}
$$

while the higher components

$$
\begin{aligned}
J_{10}=-\bar{z} \frac{\delta \Gamma_{0}}{\delta \bar{\xi}}-2\left(z K_{z}-\bar{z} K_{\bar{z}}\right)_{z} \xi+4 i\left(z g_{z}-\bar{z} g_{\bar{z}}\right) \dot{\bar{z}} \xi \\
J_{01}=z \frac{\delta \Gamma_{0}}{\delta \xi}+2\left(z K_{z}-\bar{z} K_{\bar{z}}\right)_{\bar{z}} \bar{\xi}+4 i\left(z g_{z}-\bar{z} g_{\bar{z}}\right) \dot{z} \bar{\xi} \\
J_{11}=-i\left(z \frac{\delta \Gamma_{0}}{\delta z}+\bar{z} \frac{\delta \Gamma_{0}}{\delta \bar{z}}+\xi \frac{\delta \Gamma_{0}}{\delta \xi}+\bar{\xi} \frac{\delta \Gamma_{0}}{\delta \bar{\xi}}\right) \\
+\frac{d}{d t}\left(\left(z K_{z}-\bar{z} K_{\bar{z}}\right)+2\left(z g_{z}-\bar{z} g_{\bar{z}}\right) \xi \bar{\xi}\right)
\end{aligned}
$$

do not generate any symmetries.

The $U_{J}(1)$ charge obeys the conservation equation

$$
\frac{d}{d t} j=\left(-z \frac{\delta \Gamma_{0}}{\delta z}+\bar{z} \frac{\delta \Gamma_{0}}{\delta \bar{z}}-\xi \frac{\delta \Gamma_{0}}{\delta \xi}+\bar{\xi} \frac{\delta \Gamma_{0}}{\delta \bar{\xi}}\right)-i \delta^{U(1)} L,
$$

where the $U_{J}(1)$ variations of the component coordinates are

$$
\begin{array}{ll}
\delta^{U(1)} z=i z \quad & \delta^{U(1)} \bar{z}=-i \bar{z} \\
\delta^{U(1)} \xi=i \xi & , \quad \delta^{U(1)} \bar{\xi}=-i \bar{\xi} .
\end{array}
$$

The $U_{J}(1)$ variation of the component coordinate Lagrangian $L$ is

$$
\begin{aligned}
\delta^{U(1)} L= & \left(4 i \dot{\bar{z}} \dot{\bar{z}}+2 \xi \bar{\xi}\left(\dot{z} \frac{\partial}{\partial z}-\dot{\bar{z}} \frac{\partial}{\partial \bar{z}}\right)-2(\xi \dot{\bar{\xi}}-\dot{\xi} \bar{\xi})\right)\left(z g_{z}-\bar{z} g_{\bar{z}}\right) \\
& -\left(\dot{z} \frac{\partial}{\partial z}-\dot{\bar{z}} \frac{\partial}{\partial \bar{z}}+i \xi \bar{\xi} \frac{\partial^{2}}{\partial z \partial \bar{z}}\right)\left(z K_{z}-\bar{z} K_{\bar{z}}\right)
\end{aligned}
$$

which gives the structure of the $U_{J}(1)$ Ward identity. In superspace, this identity is obtained by applying the action principle to equation (3.39) yielding

$$
\hat{w}^{U(1)} \Gamma=\left(w^{U(1)}-\bar{w}^{U(1)}\right) \Gamma=\left[\frac{\partial}{\partial t} J\right] \Gamma+\left[\frac{i}{4}[D, \bar{D}] \Delta^{U(1)} \mathcal{L}\right] \Gamma .
$$


Integrating over time yields the variation of the effective action

$$
\delta^{U(1)} \Gamma=\int d t \hat{w}^{U(1)} \Gamma=\left[\frac{i}{2} \int d V \Delta^{U(1)} \mathcal{L}\right] \Gamma .
$$

Similarly, since the time translation of the supercoordinates is a covariant transformation, the Noether construction also yields a supercoordinate charge whose first component is the Hamiltonian $h$

$$
\begin{aligned}
H & =4 g \dot{\phi} \dot{\bar{\phi}}+K_{\phi \bar{\phi}} D \phi \bar{D} \bar{\phi} \\
& =h+\theta \sqrt{2} H_{10}+\bar{\theta} \sqrt{2} H_{01}+\theta \bar{\theta} H_{11} .
\end{aligned}
$$

Explicitly, the component operators are given by

$$
\begin{aligned}
h & =4 g \dot{\bar{z}} \dot{\bar{z}}+2 K_{z \bar{z}} \xi \bar{\xi} \\
H_{10} & =\dot{\bar{z}} \frac{\delta \Gamma_{0}}{\delta \bar{\xi}} \\
H_{01} & =\dot{z} \frac{\delta \Gamma_{0}}{\delta \xi} \\
H_{11} & =\left(-i \dot{z} \frac{\delta \Gamma_{0}}{\delta z}+i \dot{\bar{z}} \frac{\delta \Gamma_{0}}{\delta \bar{z}}-i \dot{\xi} \frac{\delta \Gamma_{0}}{\delta \xi}+i \dot{\bar{\xi}} \frac{\delta \Gamma_{0}}{\delta \bar{\xi}}\right) .
\end{aligned}
$$

Time translations are generated by $h$ which satisfies the conservation law

$$
\frac{d}{d t} h=-\left(\dot{z} \frac{\delta \Gamma_{0}}{\delta z}+\dot{\bar{z}} \frac{\delta \Gamma_{0}}{\delta \bar{z}}+\dot{\xi} \frac{\delta \Gamma_{0}}{\delta \xi}+\dot{\bar{\xi}} \frac{\delta \Gamma_{0}}{\delta \bar{\xi}}\right) .
$$

Equivalently, applying the quantum action principle to equation (3.36) yields the local Ward identity

$$
\hat{w}^{H} \Gamma=\left(w^{H}-\bar{w}^{H}\right) \Gamma=-i \frac{\partial}{\partial t} H
$$

while integrating over time yields the time translation invariance of the effective action

$$
\delta^{H} \Gamma=\int d t \hat{w}^{H} \Gamma=0
$$

The remaining superconformal transformations do not (anti-)commute with the SUSY charges. However, since both the $R$ and SUSY transformations commute with $H$, the quasisupercoordinate construction will lead to a combination of charges that also forms a supercoordinate since they act as constant supercoordinates. Specifically, the quasi-supercoordinate combination of variations corresponding to $\hat{R}$ is nothing but the SUSY covariant $U(1)$ transformation 


$$
\begin{aligned}
i[\hat{R}, \tilde{\phi}] & =i[R-i \theta Q+i \bar{Q} \bar{\theta}-2 \theta \bar{\theta} H, \tilde{\phi}] \\
& =\delta^{R} \tilde{\phi}-i \theta \delta^{Q} \tilde{\phi}-i \bar{\theta} \delta^{\bar{Q}} \tilde{\phi}-2 \theta \bar{\theta} \delta^{H} \tilde{\phi} \\
& =n_{\phi} \delta^{U(1)} \tilde{\phi}=\left\{\begin{array}{c}
i n_{\phi} \phi \\
-i n_{\phi} \bar{\phi}
\end{array}\right.
\end{aligned}
$$

where $\tilde{\phi}$ is either $\phi$ or $\bar{\phi}$. The Noether procedure applied to the $U(1)$ symmetry yields a supercoordinate charge as given in equation (3.39) and (4.1) above. Expanding the $U(1)$ variation of the supercoordinates, multiplying by the corresponding derivative of the action, applying the covariant derivatives and inserting it all into one- particle irreducible functions yields the relation

$$
\begin{aligned}
-i\left[w^{R} \Gamma+i \theta w^{Q} \Gamma\right. & \left.+i \bar{\theta} w^{\bar{Q}} \Gamma-2 \theta \bar{\theta} w^{H} \Gamma\right]=\left[D\left(\delta^{R} \phi \frac{\delta \Gamma_{0}}{\delta \phi}\right)\right] \Gamma \\
& +\left[i \theta D\left(\delta^{Q} \phi \frac{\delta \Gamma_{0}}{\delta \phi}\right)\right] \Gamma+\left[i \bar{\theta} D\left(\delta^{\bar{Q}} \phi \frac{\delta \Gamma_{0}}{\delta \phi}\right)\right] \Gamma+\left[-2 \theta \bar{\theta} D\left(\delta^{H} \phi \frac{\delta \Gamma_{0}}{\delta \phi}\right)\right] \Gamma \\
& =\left[D\left(n_{\phi} \delta^{U(1)} \phi \frac{\delta \Gamma_{0}}{\delta \phi}\right)\right] \Gamma+\left[i D \phi \frac{\delta \Gamma_{0}}{\delta \phi}\right] \Gamma,
\end{aligned}
$$

where the last equality was obtained upon application of the action principle. A similar expression is obtained for the antichiral supercoordinate variations yielding

$$
\begin{aligned}
-i\left(\bar{w}^{R} \Gamma+i \theta \bar{w}^{Q} \Gamma\right. & \left.+i \bar{\theta} \bar{w}^{\bar{Q}} \Gamma-2 \theta \bar{\theta} \bar{w}^{H} \Gamma\right)=\left[\bar{D}\left(\delta^{R} \bar{\phi} \frac{\delta \Gamma_{0}}{\delta \bar{\phi}}\right)\right] \Gamma \\
& +\left[i \theta \bar{D}\left(\delta^{Q} \bar{\phi} \frac{\delta \Gamma_{0}}{\delta \bar{\phi}}\right)\right] \Gamma+\left[i \bar{\theta} \bar{D}\left(\delta^{\bar{Q}} \bar{\phi} \frac{\delta \Gamma_{0}}{\delta \bar{\phi}}\right)\right] \Gamma+\left[-2 \theta \bar{\theta} \bar{D}\left(\delta^{H} \bar{\phi} \frac{\delta \Gamma_{0}}{\delta \bar{\phi}}\right)\right] \Gamma \\
& =\bar{D}\left[n_{\phi} \delta^{U(1)} \bar{\phi} \frac{\delta \Gamma_{0}}{\delta \bar{\phi}}\right] \Gamma-i\left[\bar{D} \bar{\phi} \frac{\delta \Gamma_{0}}{\delta \bar{\phi}}\right] \Gamma .
\end{aligned}
$$

Taking the difference of these equations and recalling the conservation equations for the $U_{J}(1)$ and $R_{0}$ charges, equations (3.39)- (3.42), the supercoordinate charge $\hat{R}=R=\left(n_{\phi} J+\right.$ $\left.R_{0}\right)$ is obtained and seen to obey the Ward identity equation

$$
\begin{aligned}
\hat{w}^{R} \Gamma & =\left(w^{R}-\bar{w}^{R}\right) \Gamma+i \theta\left(w^{Q}-\bar{w}^{Q}\right) \Gamma+i \bar{\theta}\left(w^{\bar{Q}}-\bar{w}^{\bar{Q}}\right) \Gamma-2 \theta \bar{\theta}\left(w^{H}-\bar{w}^{H}\right) \Gamma \\
& =\left[-\frac{\partial}{\partial t} \hat{R}\right] \Gamma+\left[-\frac{i}{2}[D, \bar{D}] \frac{n_{\phi}}{2} \Delta^{U(1)} \mathcal{L}\right] \Gamma
\end{aligned}
$$

A special case is obtained for $n_{\phi}=0$ where the supercoordinate of charges $R$ reduces to the conserved $R_{0}$ charge supercoordinate. It obeys the Ward identity

$$
\hat{w}^{R_{0}} \Gamma=\frac{\partial}{\partial t} R_{0}
$$

where the Ward identity operator is given by $\hat{w}^{R_{0}}=w^{R_{0}}-\bar{w}^{R_{0}}$ with 


$$
\begin{aligned}
w^{R_{0}} & =i D \phi \frac{\delta}{\delta \phi} \\
\bar{w}^{R_{0}} & =-i \bar{D} \bar{\phi} \frac{\delta}{\delta \bar{\phi}} .
\end{aligned}
$$

As is discussed in Appendix B, the quasi-supercoordinate $\hat{Q}$ and $\hat{\bar{Q}}$ beginning with $Q$ and $\bar{Q}$, respectively, can be constructed as

$$
\begin{aligned}
i[\hat{Q}, \tilde{\phi}] & =i[Q-2 i \bar{\theta} H, \tilde{\phi}] \\
& =\delta^{Q} \tilde{\phi}-2 i \bar{\theta} \delta^{H} \tilde{\phi}=D \tilde{\phi} \\
i[\hat{\bar{Q}}, \tilde{\phi}] & =i[\bar{Q}+2 i \theta H, \tilde{\phi}] \\
& =\delta^{\bar{Q}} \tilde{\phi}+2 i \theta \delta^{H} \tilde{\phi}=\bar{D} \tilde{\phi}
\end{aligned}
$$

Once again there are two equivalent directions along which to proceed. Since $D$ and $\bar{D}$ are covariant derivatives, the Noether construction leads to supercoordinate charges $Q$ and $\bar{Q}$. It is found that

$$
\hat{Q}=\mathcal{Q}_{I}^{Q}-2 i \bar{\theta} \mathcal{Q}_{I}^{H}=-Q,
$$

with the conservation equation

$$
\hat{w}^{Q} \Gamma=\left(w^{Q}-\bar{w}^{Q}\right) \Gamma+2 i \bar{\theta}\left(w^{H}-\bar{w}^{H}\right) \Gamma=\left[i \frac{\partial Q}{\partial t}\right] \Gamma .
$$

Likewise

$$
\hat{\bar{Q}}=\mathcal{Q}_{I}^{\bar{Q}}+2 i \theta \mathcal{Q}_{I}^{H}=-\bar{Q},
$$

with the corresponding conservation equation

$$
\hat{w}^{\bar{Q}} \Gamma=\left(w^{\bar{Q}}-\bar{w}^{\bar{Q}}\right) \Gamma-2 i \theta\left(w^{H}-\bar{w}^{H}\right) \Gamma=\left[i \frac{\partial \bar{Q}}{\partial t}\right] \Gamma .
$$

Equivalently, the Euler-Lagrange equations and hence the Ward identity operators can be used directly in order to derive the supercoordinate charges $Q$ and $\bar{Q}$. Taking covariant derivatives of the variations (4.21) gives

$$
\begin{aligned}
& D\left(\delta^{Q} \phi \frac{\delta \Gamma_{0}}{\delta \phi}\right)+2 i \bar{\theta} D\left(\delta^{H} \phi \frac{\delta \Gamma_{0}}{\delta \phi}\right)=D\left(D \phi \frac{\delta \Gamma_{0}}{\delta \phi}\right) \\
& \bar{D}\left(\delta^{Q} \bar{\phi} \frac{\delta \Gamma_{0}}{\delta \bar{\phi}}\right)+2 i \bar{\theta} \bar{D}\left(\delta^{H} \bar{\phi} \frac{\delta \Gamma_{0}}{\delta \bar{\phi}}\right)=-2 i \dot{\bar{\phi}} \frac{\delta \Gamma_{0}}{\delta \bar{\phi}} .
\end{aligned}
$$

Inserting these identities into the 1-PI functions and using the action principle, yields, after their subtraction, 


$$
\begin{aligned}
\hat{w}^{Q} \Gamma & =\left(w^{Q}-\bar{w}^{Q}\right) \Gamma+2 i \bar{\theta}\left(w^{H}-\bar{w}^{H}\right) \Gamma \\
& =\left[D\left(D \phi \frac{\delta \Gamma_{0}}{\delta \phi}\right)\right] \Gamma+\left[2 i \overline{\bar{\phi}} \frac{\delta \Gamma_{0}}{\delta \bar{\phi}}\right] \Gamma \\
& =\left[i \frac{\partial Q}{\partial t}\right] \Gamma
\end{aligned}
$$

where the identities in equations (3.37) and (3.38) have also been exploited. In a similar fashion, the identities lead to

$$
\begin{aligned}
& D\left(\delta^{\bar{Q}} \phi \frac{\delta \Gamma_{0}}{\delta \phi}\right)-2 i \theta D\left(\delta^{H} \phi \frac{\delta \Gamma_{0}}{\delta \phi}\right)=-2 i \dot{\phi} \frac{\delta \Gamma_{0}}{\delta \phi} \\
& \bar{D}\left(\delta^{\bar{Q}} \bar{\phi} \frac{\delta \Gamma_{0}}{\delta \bar{\phi}}\right)-2 i \theta \bar{D}\left(\delta^{H} \bar{\phi} \frac{\delta \Gamma_{0}}{\delta \bar{\phi}}\right)=\left[\bar{D}\left(\bar{D} \bar{\phi} \frac{\delta \Gamma_{0}}{\delta \bar{\phi}}\right)\right] .
\end{aligned}
$$

Inserting these identities into the 1-PI functions and using the action principle produces

$$
\begin{aligned}
\hat{w}^{\bar{Q}} \Gamma & =\left(w^{\bar{Q}}-\bar{w}^{\bar{Q}}\right) \Gamma-2 i \theta\left(w^{H}-\bar{w}^{H}\right) \Gamma \\
& =\left[-\bar{D}\left(\bar{D} \bar{\phi} \frac{\delta \Gamma_{0}}{\delta \bar{\phi}}\right)\right] \Gamma+\left[-2 i \dot{\phi} \frac{\delta \Gamma_{0}}{\delta \phi}\right] \Gamma \\
& =\left[i \frac{\partial \bar{Q}}{\partial t}\right] \Gamma
\end{aligned}
$$

where the identities in equations (3.37) and (3.38) have again been employed.

Having considered combinations of transformations that are covariant variations, either being the supercoordinate itself or a covariant derivative of the supercoordinate, the Noether construction led to a multiplet of charges that was a supercoordinate. Indeed, the multiplet corresponding to the $R_{0}$ transformation contains the supersymmetry charges and the Hamiltonian among its components. Specifically expressing the supercharge $R_{0}$ in terms of its component charges gives

$$
\begin{aligned}
R_{0} & =-2 i g D \phi \bar{D} \bar{\phi} \\
& =r_{0}+i \theta \sqrt{2} q-i \sqrt{2} \bar{q} \bar{\theta}+\theta \bar{\theta}\left(2 i h+i\left(\xi \frac{\delta \Gamma_{0}}{\delta \xi}+\bar{\xi} \frac{\delta \Gamma_{0}}{\delta \bar{\xi}}\right)\right)
\end{aligned}
$$

with the component charges given in terms of the component coordinates as

$$
\begin{aligned}
r_{0} & =-4 i g \xi \bar{\xi} \\
q & =-4 g \dot{\bar{z}} \xi \quad ; \quad \bar{q}=4 g \dot{z} \bar{\xi} \\
h & =4 g \dot{z} \dot{\bar{z}}+2 K_{z \bar{z}} \xi \bar{\xi} .
\end{aligned}
$$

The corresponding component charge conservation equations are

$$
\left[\frac{\partial}{\partial t} r_{0}\right] \Gamma=\left[i\left(-\xi \frac{\delta}{\delta \xi}+\bar{\xi} \frac{\delta}{\delta \bar{\xi}}\right)\right] \Gamma
$$




$$
\begin{aligned}
& {\left[\frac{\partial}{\partial t} q\right] \Gamma=\left[i\left(\xi \frac{\delta}{\delta z}-2 i \dot{\bar{z}} \frac{\delta}{\delta \bar{\xi}}\right)\right] \Gamma} \\
& {\left[\frac{\partial}{\partial t} \bar{q}\right] \Gamma=\left[i\left(-\bar{\xi} \frac{\delta}{\delta \bar{z}}+2 i \dot{z} \frac{\delta}{\delta \xi}\right)\right] \Gamma} \\
& {\left[\frac{\partial}{\partial t} h\right] \Gamma=\left[i\left(\dot{z} \frac{\delta}{\delta z}+\dot{\bar{z}} \frac{\delta}{\delta \bar{z}}+\dot{\xi} \frac{\delta}{\delta \xi}+\dot{\bar{\xi}} \frac{\delta}{\delta \bar{\xi}}\right)\right] \Gamma,}
\end{aligned}
$$

where $r_{0}$ is the $R$ charge for phase transformations involving the Grassmann coordinates only, while $q$ and $\bar{q}$ are the SUSY charges and $h$ the Hamiltonian.

The remainder of the superconformal charges can only be promoted to quasi- supercoordinates. For example, following the procedure outlined in Appendix B, the dilatation transformations spawn the quasi-supercoordinate of transformations

$$
\begin{aligned}
i[\hat{D}, \tilde{\phi}] & =i\left[D-\frac{1}{2} \theta Q-\frac{1}{2} \bar{Q} \bar{\theta}, \tilde{\phi}\right] \\
& =\delta^{D} \tilde{\phi}-\frac{1}{2} \theta \delta^{Q} \tilde{\phi}+\frac{1}{2} \bar{\theta} \delta^{\bar{Q}} \tilde{\phi} \\
& =d_{\phi} \tilde{\phi}+t \partial_{t} \tilde{\phi},
\end{aligned}
$$

which is not SUSY covariant due to the explicit time dependence. The Ward identity operator corresponding to this transformation is obtained by taking covariant derivatives of the above combination of variations. For the chiral supercoordinate, it takes the form

$$
\begin{aligned}
-i w^{\hat{D}} \Gamma & =\left[D\left(\delta^{D} \phi \frac{\delta \Gamma_{0}}{\delta \phi}\right)\right] \Gamma+\left[\frac{1}{2} \theta D\left(\delta^{Q} \phi \frac{\delta \Gamma_{0}}{\delta \phi}\right)\right] \Gamma+\left[-\frac{1}{2} \bar{\theta} D\left(\delta^{\bar{Q}} \phi \frac{\delta \Gamma_{0}}{\delta \phi}\right)\right] \Gamma \\
& =\left[\frac{1}{2} D \phi \frac{\delta \Gamma_{0}}{\delta \phi}\right] \Gamma+\left[t D\left(\dot{\phi} \frac{\delta \Gamma_{0}}{\delta \phi}\right)\right] \Gamma+\left[D\left(d_{\phi} \phi \frac{\delta \Gamma_{0}}{\delta \phi}\right)\right] \Gamma
\end{aligned}
$$

while for the transformation of the antichiral supercoordinate

$$
\begin{aligned}
-i \bar{w}^{\hat{D}} \Gamma & =\left[\bar{D}\left(\delta^{D} \bar{\phi} \frac{\delta \Gamma_{0}}{\delta \bar{\phi}}\right)\right] \Gamma+\left[\frac{1}{2} \theta \bar{D}\left(\delta^{Q} \bar{\phi} \frac{\delta \Gamma_{0}}{\delta \bar{\phi}}\right)\right] \Gamma+\left[-\frac{1}{2} \bar{\theta} \bar{D}\left(\delta^{\bar{Q}} \bar{\phi} \frac{\delta \Gamma_{0}}{\delta \bar{\phi}}\right)\right] \Gamma \\
& =\left[\frac{1}{2} \bar{D} \bar{\phi} \frac{\delta \Gamma_{0}}{\delta \bar{\phi}}\right] \Gamma+\left[t \bar{D}\left(\dot{\bar{\phi}} \frac{\delta \Gamma_{0}}{\delta \bar{\phi}}\right)\right] \Gamma+\left[\bar{D}\left(d_{\phi} \bar{\phi} \frac{\delta \Gamma_{0}}{\delta \bar{\phi}}\right)\right] \Gamma .
\end{aligned}
$$

Hence, the Ward identity operator conservation equation becomes

$$
\begin{aligned}
\hat{w}^{D} \Gamma & =\left(w^{\hat{D}}-\bar{w}^{\hat{D}}\right) \Gamma \\
& =\left[-i \frac{\partial}{\partial t} \hat{D}\right] \Gamma+\left[-\frac{i}{2}[D, \bar{D}] \Delta_{d_{\phi}}^{D} \mathcal{L}\right] \Gamma
\end{aligned}
$$

where the dilatation quasi-supercoordinate of charges $\hat{D}$ is

$$
\hat{D}=t H+d_{\phi} U
$$


Here $U$ is the global Weyl scaling charge given by the supercoordinate

$$
U=2 i\left(\mathcal{Q}^{N}+\mathcal{Q}^{\bar{N}}\right),
$$

while the variation of the Lagrangian is given by

$$
\begin{aligned}
\Delta^{D} d_{\phi} \mathcal{L} & =-i \frac{d_{\phi}}{2} \delta^{U} \mathcal{L}+\frac{i}{2} R_{0} \\
& =\frac{i}{4}\left(-4 i d_{\phi} \delta^{N} \mathcal{L}+R_{0}\right)+\frac{i}{4}\left(-4 i d_{\phi} \delta^{\bar{N}} \mathcal{L}+R_{0}\right) .
\end{aligned}
$$

This is the same result obtained previously by combining the Noether charges to directly form the dilatation quasi-supercoordinate $\hat{D}$

$$
\begin{aligned}
\hat{D} & =\mathcal{Q}_{I}^{D}+\frac{1}{2} \theta \mathcal{Q}_{I}^{Q}-\frac{1}{2} \bar{\theta} \mathcal{Q}_{I}^{\bar{Q}} \\
& =t H+d_{\phi} U .
\end{aligned}
$$

The conformal SUSY Noether charges can be used as the initial components of the conformal SUSY quasi-supercoordinates of charges according to

$$
\begin{aligned}
\hat{\bar{S}} & =2 i\left(\mathcal{Q}_{I}^{\bar{S}}-\bar{\theta}\left(2 i \mathcal{Q}_{I}^{D}-\mathcal{Q}_{I}^{R}\right)\right)+i \theta \bar{\theta} \mathcal{Q}_{I}^{Q} \\
& =t Q+4 g D \phi \bar{\phi}+2 \phi g_{\phi} D \phi \bar{\phi} \\
& =t Q+2 D(g \phi \bar{\phi})+2 i \bar{T} \\
& =t Q+4 i \bar{T}+2 \bar{Y} \\
\hat{S} & =2 i\left(\mathcal{Q}_{I}^{S}+\theta\left(2 i \mathcal{Q}_{I}^{D}+\mathcal{Q}_{I}^{R}\right)\right)-i \theta \bar{\theta} \mathcal{Q}_{I}^{\bar{Q}} \\
& =t \bar{Q}+4 g \phi \bar{D} \bar{\phi}+2 \bar{\phi} g_{\bar{\phi}} \phi \bar{D} \bar{\phi} \\
& =t \bar{Q}+2 \bar{D}(g \phi \bar{\phi})+2 i T \\
& =t \bar{Q}+4 i T+2 Y .
\end{aligned}
$$

These correspond to the variations

$$
\begin{aligned}
i[\hat{\bar{S}}, \tilde{\phi}] & =i[\bar{S}+\bar{\theta}(2 i D+R)+i \theta \bar{\theta} Q, \tilde{\phi}] \\
& =\delta^{\bar{S}} \tilde{\phi}+\bar{\theta}\left(2 i \delta^{D} \tilde{\phi}+\delta^{R} \tilde{\phi}\right)+i \theta \bar{\theta} \delta^{Q} \tilde{\phi} \\
& =-t D \tilde{\phi} \\
i[\hat{S}, \tilde{\phi}] & =i[S+\theta(-2 i D+R)-i \theta \bar{\theta} \bar{Q}, \tilde{\phi}] \\
& =\delta^{S} \tilde{\phi}+\theta\left(-2 i \delta^{D} \tilde{\phi}+\delta^{R} \tilde{\phi}\right)-i \theta \bar{\theta} \delta^{\bar{Q}} \\
& =-t \bar{D} \tilde{\phi} .
\end{aligned}
$$

Applying the conservation equations for the individual Noether charges gives the quasisupercoordinate conservation equations 


$$
\begin{aligned}
& \hat{w}^{\bar{S}} \Gamma=\left[i \frac{\partial}{\partial t} \hat{\bar{S}}\right] \Gamma+\left[\frac{i}{2}[D, \bar{D}] \Delta^{\bar{S}} \mathcal{L}\right] \Gamma+\left[-\bar{\theta}\left(\frac{i}{2}[D, \bar{D}]\left(2 i \Delta^{D} \mathcal{L}-\Delta^{R} \mathcal{L}\right)\right)\right] \Gamma \\
& \hat{w}^{S} \Gamma=\left[i \frac{\partial}{\partial t} \hat{S}\right] \Gamma+\left[\frac{i}{2}[D, \bar{D}] \Delta^{S} \mathcal{L}\right] \Gamma+\left[\theta\left(\frac{i}{2}[D, \bar{D}]\left(2 i \Delta^{D} \mathcal{L}+\Delta^{R} \mathcal{L}\right)\right)\right] \Gamma
\end{aligned}
$$

where the Ward identity operators are

$$
\begin{aligned}
& \hat{w}^{\bar{S}}=w^{\hat{\bar{S}}}-\bar{w}^{\hat{\bar{S}}} \\
& \hat{w}^{S}=w^{\hat{S}}-\bar{w}^{\hat{S}},
\end{aligned}
$$

with

$$
\begin{aligned}
& w^{\bar{S}}=D\left(\delta^{\bar{S}} \phi \frac{\delta \Gamma_{0}}{\delta \phi}\right)-\bar{\theta} D\left(\left(2 i \delta^{D} \phi \frac{\delta \Gamma_{0}}{\delta \phi}+\delta^{R} \phi \frac{\delta \Gamma_{0}}{\delta \phi}\right)\right)+i \theta \bar{\theta} D\left(\delta^{Q} \phi \frac{\delta \Gamma_{0}}{\delta \phi}\right) \\
& \bar{w}^{\bar{S}}=\bar{D}\left(\delta^{\bar{S}} \bar{\phi} \frac{\delta \Gamma_{0}}{\delta \bar{\phi}}\right)-\bar{\theta} \bar{D}\left(\left(2 i \delta^{D} \bar{\phi} \frac{\delta \Gamma_{0}}{\delta \bar{\phi}}+\delta^{R} \bar{\phi} \frac{\delta \Gamma_{0}}{\delta \bar{\phi}}\right)\right)+i \theta \bar{\theta} \bar{D}\left(\delta^{Q} \bar{\phi} \frac{\delta \Gamma_{0}}{\delta \bar{\phi}}\right) \\
& w^{S}=D\left(\delta^{S} \phi \frac{\delta \Gamma_{0}}{\delta \phi}\right)-\theta D\left(\left(-2 i \delta^{D} \phi \frac{\delta \Gamma_{0}}{\delta \phi}+\delta^{R} \phi \frac{\delta \Gamma_{0}}{\delta \phi}\right)\right)-i \theta \bar{\theta} D\left(\delta^{\bar{Q}} \phi \frac{\delta \Gamma_{0}}{\delta \phi}\right) \\
& \bar{w}^{S}=\bar{D}\left(\delta^{S} \bar{\phi} \frac{\delta \Gamma_{0}}{\delta \bar{\phi}}\right)-\theta \bar{D}\left(\left(-2 i \delta^{D} \bar{\phi} \frac{\delta \Gamma_{0}}{\delta \bar{\phi}}+\delta^{R} \bar{\phi} \frac{\delta \Gamma_{0}}{\delta \bar{\phi}}\right)\right)-i \theta \bar{\theta} \bar{D}\left(\delta^{\bar{Q}} \bar{\phi} \frac{\delta \Gamma_{0}}{\delta \bar{\phi}}\right) .
\end{aligned}
$$

Utilizing the identities

$$
\begin{aligned}
\bar{\theta}[D, \bar{D}] & =[D, \bar{D}] \bar{\theta}+2 D \\
\theta[D, \bar{D}] & =[D, \bar{D}] \theta+2 \bar{D} \\
\theta \bar{\theta}[D, \bar{D}] & =[D, \bar{D}] \theta \bar{\theta}-[1-2 \theta \bar{D}+2 \bar{\theta} \bar{D}]
\end{aligned}
$$

and employing the canonical $R$ and scale weights, $n_{\phi}=-2 d_{\phi}=1=-n_{\bar{\phi}}$, these take the form

$$
\begin{aligned}
-i \hat{w}^{\bar{S}} \Gamma & =\left[\frac{\partial}{\partial t} \hat{\bar{S}}\right] \Gamma+\left[\frac{1}{2}[D, \bar{D}]\left(\Delta^{\bar{S}} \mathcal{L}-\bar{\theta}\left(2 i \Delta^{D} \mathcal{L}-\Delta^{R} \mathcal{L}\right)\right)\right] \Gamma-\left[D\left(2 i \Delta^{D} \mathcal{L}-\Delta^{R} \mathcal{L}\right)\right] \Gamma \\
& =\left[\frac{\partial}{\partial t} \hat{\bar{S}}\right] \Gamma+\left[\frac{1}{2}[D, \bar{D}](2 i \bar{Y})\right] \Gamma-\left[D\left(2 i \bar{\phi} K_{\bar{\phi}}+2 i \bar{\phi} g_{\bar{\phi}} D \phi \bar{D} \bar{\phi}\right)\right] \Gamma \\
& =\left[\frac{\partial}{\partial t}[\hat{\bar{S}}-2 \bar{Y}]\right] \Gamma-\left[D\left(2 i \delta^{\bar{N}} \mathcal{L}+R_{0}\right)\right] \Gamma \\
& =\left[\frac{\partial}{\partial t}(t Q)\right] \Gamma-[Q] \Gamma+2 i\left[\bar{\phi} \frac{\delta \Gamma_{0}}{\delta \bar{\phi}}\right] \Gamma .
\end{aligned}
$$

On the other hand, for general $R$ and scale weights, the Ward identity becomes

$$
-i \hat{w}^{\bar{S}} \Gamma=\left[\frac{\partial}{\partial t}(t Q)\right] \Gamma-[Q] \Gamma+\left[i\left(n_{\phi}-2 d_{\phi}\right)\left(\bar{\phi} \frac{\delta \Gamma_{0}}{\delta \bar{\phi}}\right)\right] \Gamma
$$

In analogous fashion, for canonical $R$ and scale weights, 


$$
\begin{aligned}
-i \hat{w}^{S} \Gamma & =\left[\frac{\partial}{\partial t} \hat{S}\right] \Gamma+\left[\frac{1}{2}[D, \bar{D}]\left(\Delta^{S} \mathcal{L}+\theta\left(2 i \Delta^{D} \mathcal{L}+\Delta^{R} \mathcal{L}\right)\right)\right] \Gamma+\left[\bar{D}\left(2 i \Delta^{D} \mathcal{L}+\Delta^{R} \mathcal{L}\right)\right] \Gamma \\
& =\left[\frac{\partial}{\partial t} \hat{S}\right] \Gamma+\left[\frac{1}{2}[D, \bar{D}](-2 i Y)\right] \Gamma+\left[\bar{D}\left(2 i \phi K_{\phi}+2 i \phi g_{\phi} D \phi \bar{D} \bar{\phi}\right)\right] \Gamma \\
& =\left[\frac{\partial}{\partial t}(\hat{S}-2 Y)\right] \Gamma+\left[\bar{D}\left(2 i \delta^{N} \mathcal{L}+R_{0}\right)\right] \Gamma \\
& =\left[\frac{\partial}{\partial t}(t \bar{Q})\right] \Gamma+[-\bar{Q}] \Gamma+\left[-2 i\left(\phi \frac{\delta \Gamma_{0}}{\delta \phi}\right)\right] \Gamma
\end{aligned}
$$

while for general $R$ and scale weights, the Ward identity takes the form

$$
-i \hat{w}^{S} \Gamma=\left[\frac{\partial}{\partial t}(t \bar{Q})\right] \Gamma+[-\bar{Q}] \Gamma+\left[-i\left(n_{\phi}-2 d_{\phi}\right)\left(\phi \frac{\delta \Gamma_{0}}{\delta \phi}\right)\right] \Gamma
$$

Finally, the conformal transformation yields the quasi-supercoordinate variation

$$
\begin{aligned}
i[\hat{K}, \tilde{\phi}] & =i[K+\theta \bar{S}+S \bar{\theta}+\theta \bar{\theta} R, \tilde{\phi}] \\
& =\delta^{K} \tilde{\phi}+\theta \delta^{\bar{S}} \tilde{\phi}-\bar{\theta} \delta^{S} \tilde{\phi}+\theta \bar{\theta} \delta^{R} \tilde{\phi} \\
& =2 t d_{\phi} \tilde{\phi}+t^{2} \partial_{t} \tilde{\phi}
\end{aligned}
$$

along with the quasi-supercoordinate of Noether charges

$$
\begin{aligned}
\hat{K} & =2 i\left(-\mathcal{Q}_{I}^{K}-\theta \mathcal{Q}_{I}^{\bar{S}}+\bar{\theta} \mathcal{Q}_{I}^{S}-\theta \bar{\theta} \mathcal{Q}_{I}^{R}\right) \\
& =t(U-t H)-4 g \phi \bar{\phi}
\end{aligned}
$$

The conservation equation yields the conformal Ward identity

$$
\begin{aligned}
-i \hat{w}^{K} \Gamma= & {\left[\frac{\partial}{\partial t} \hat{K}\right] \Gamma-\left[\frac{1}{2}[D, \bar{D}] \Delta^{K} \mathcal{L}\right] \Gamma-\frac{1}{2} \theta\left[[D, \bar{D}] \Delta^{\bar{S}} \mathcal{L}\right] \Gamma+\frac{1}{2} \bar{\theta}\left[[D, \bar{D}] \Delta^{S} \mathcal{L}\right] \Gamma } \\
& -\frac{1}{2} \theta \bar{\theta}\left[[D, \bar{D}] \Delta^{R} \mathcal{L}\right] \Gamma \\
= & {\left[\frac{\partial}{\partial t} \hat{K}\right] \Gamma-\left[\frac{1}{2}[D, \bar{D}]\left(\Delta^{K} \mathcal{L}+\theta \Delta^{\bar{S}} \mathcal{L}-\bar{\theta} \Delta^{S} \mathcal{L}+\theta \bar{\theta} \Delta^{R} \mathcal{L}\right)\right] \Gamma } \\
& -\left[\bar{D} \Delta^{\bar{S}} \mathcal{L}\right] \Gamma+\left[D \Delta^{S} \mathcal{L}\right] \Gamma+\left[(1-\theta D+\bar{\theta} \bar{D}) \Delta^{R} \mathcal{L}\right] \Gamma
\end{aligned}
$$

where the conformal Ward identity operator is $\hat{w}^{K}=w^{K}-\bar{w}^{K}$ with the chiral and antichiral Ward identity operators given by

$$
\begin{aligned}
& w^{K}=D\left[\delta^{K} \phi \frac{\delta \Gamma_{0}}{\delta \phi}\right]-\theta D\left[\delta^{\bar{S}} \phi \frac{\delta \Gamma_{0}}{\delta \phi}\right]+\bar{\theta} D\left[\delta^{S} \phi \frac{\delta \Gamma_{0}}{\delta \phi}\right]+\theta \bar{\theta} D\left[\delta^{R} \phi \frac{\delta \Gamma_{0}}{\delta \phi}\right] \\
& \bar{w}^{K}=\bar{D}\left[\delta^{K} \bar{\phi} \frac{\delta \Gamma_{0}}{\delta \bar{\phi}}\right]-\theta \bar{D}\left[\delta^{\bar{S}} \bar{\phi} \frac{\delta \Gamma_{0}}{\delta \bar{\phi}}\right]+\bar{\theta} \bar{D}\left[\delta^{S} \bar{\phi} \frac{\delta \Gamma_{0}}{\delta \bar{\phi}}\right]+\theta \bar{\theta} \bar{D}\left[\delta^{R} \bar{\phi} \frac{\delta \Gamma_{0}}{\delta \bar{\phi}}\right]
\end{aligned}
$$

Substituting the expressions for the variations of the Lagrangian from section 3 and exploiting the identity 


$$
[D, \bar{D}] t=t[D, \bar{D}]-2 i \theta D-2 i \bar{\theta} \bar{D},
$$

the conformal Ward identity is obtained as

$$
\begin{aligned}
-i \hat{w}^{K} \Gamma & =\left[\frac{\partial}{\partial t} \hat{K}\right] \Gamma+\left[-t \frac{i}{4}[D, \bar{D}]\left(\delta^{U} \mathcal{L}+2 R_{0}\right)\right] \Gamma+[-2 i(D Y+\bar{D} \bar{Y})] \Gamma+\left[-\frac{1}{2} \Delta^{U(1)} \mathcal{L}\right] \Gamma \\
& =\left[\frac{\partial}{\partial t}(\hat{K}+4 g \phi \bar{\phi})\right] \Gamma+[-U] \Gamma+\left[-t \frac{i}{4}[D, \bar{D}]\left(\delta^{U} \mathcal{L}+2 R_{0}\right)\right] \Gamma \\
& =\left[\frac{\partial}{\partial t}\left(t U-t^{2} H\right)\right] \Gamma+[-U] \Gamma+\left[-t \frac{i}{4}[D, \bar{D}]\left(\delta^{U} \mathcal{L}+2 R_{0}\right)\right] \Gamma .
\end{aligned}
$$

In summary, the (quasi-)supercoordinates of charges for the superconformal group are given in terms of the Hamiltonian and SUSY charge supercoordinates, which are components of the $R$ symmetry charge, and the charges associated with the chiral and antichiral global Weyl scaling of the supercoordinates. In addition, the breaking of the superconformal symmetries is given in terms of the breaking of these global Weyl scaling symmetries and the additional $U(1) J$ symmetry of the Lagrangian. In particular, the symmetries and conservation equations can be summarized as the Ward identities for the effective action: 


\begin{tabular}{|c|c|c|}
\hline Symmetry & Charge & Ward Identity \\
\hline$U(1)$ & $J$ & $\delta^{U(1)} \Gamma=\left[\frac{1}{2} \int d V \Delta^{U_{J}(1)} \mathcal{L}\right] \Gamma$ \\
\hline Time Translation & $H$ & $\delta^{H} \Gamma=0$ \\
\hline \multirow[t]{2}{*}{ Supersymmetry } & $Q$ & $\hat{\delta}^{Q} \Gamma=\left(\delta^{Q}+2 i \bar{\theta} \delta^{H}\right) \Gamma=0$ \\
\hline & $\bar{Q}$ & $\hat{\delta}^{\bar{Q}} \Gamma=\left(\delta^{\bar{Q}}-2 i \theta \delta^{H}\right) \Gamma=0$ \\
\hline$R$ Transformation & $R$ & $\begin{aligned} \hat{\delta}^{R} \Gamma & =\left(\delta^{R}+i \theta \delta^{Q}+i \bar{\theta} \delta^{\bar{Q}}-2 \theta \bar{\theta} \delta^{H}\right) \Gamma \\
& =i\left[\frac{n_{\phi}}{2} \int d V \Delta^{U(1)} \mathcal{L}\right] \Gamma\end{aligned}$ \\
\hline Dilatation & $\hat{D}$ & $\begin{aligned} \hat{\delta}^{D} \Gamma & =\left(\delta^{D}+\frac{1}{2} \theta \delta^{Q}-\frac{1}{2} \bar{\theta} \delta^{\bar{Q}}\right) \Gamma=i\left[\int d V \Delta_{d_{\phi}}^{D} \mathcal{L}\right] \Gamma \\
& =-\frac{1}{4} \int d V\left[\left(-4 i d_{\phi} \delta^{N} \mathcal{L}+R_{0}\right)+\left(-4 i d_{\phi} \delta^{\bar{N}} \mathcal{L}+R_{0}\right)\right] \Gamma\end{aligned}$ \\
\hline \multirow[t]{2}{*}{ Superconformal } & $\hat{\bar{S}}$ & $\begin{aligned} \hat{\delta}^{\bar{S}} \Gamma & =\left(\delta^{\bar{S}}-\bar{\theta}\left[2 i \delta^{D}+\delta^{R}\right]+i \theta \bar{\theta} \delta^{Q}\right) \Gamma \\
& =-i \int d S\left[-4 i d_{\phi} \delta^{\bar{N}} \mathcal{L}+R_{0}\right] \Gamma\end{aligned}$ \\
\hline & $\hat{S}$ & $\begin{aligned} \hat{\delta}^{S} \Gamma & =\left(\delta^{S}-\theta\left[-2 i \delta^{D}+\delta^{R}\right]-i \theta \bar{\theta} \delta^{\bar{Q}}\right) \Gamma \\
& =-i \int d \bar{S}\left[-4 i d_{\phi} \delta^{N} \mathcal{L}+R_{0}\right] \Gamma\end{aligned}$ \\
\hline Conformal & $\hat{K}$ & $\begin{aligned} \hat{\delta}^{K} \Gamma & =\left(\delta^{K}-\theta \delta^{\bar{S}}+\bar{\theta} \delta^{S}+\theta \bar{\theta} \delta^{R}\right) \Gamma \\
= & i \int d V\left[2 t \Delta_{d_{\phi}}^{D} \mathcal{L}\right] \Gamma+i \int d S\left[\Delta^{S} \mathcal{L}+\theta \frac{n_{\phi}}{2} \Delta^{U(1)} \mathcal{L}\right] \Gamma \\
& +i \int d \bar{S}\left[\Delta^{\bar{S}} \mathcal{L}+\bar{\theta} \frac{n_{\phi}}{2} \Delta^{U(1)} \mathcal{L}\right] \Gamma-i \int d t\left[\frac{n_{\phi}}{2} \Delta^{U(1)} \mathcal{L}\right] \Gamma\end{aligned}$ \\
\hline
\end{tabular}

Recall that in the special case that $n_{\phi}=0$, the $R$ charge reduces to the conserved $R_{0}$ charge:

$$
\begin{aligned}
R_{0} & =-2 i g D \phi \bar{D} \bar{\phi} \\
D R_{0} & =Q
\end{aligned}
$$




$$
\begin{aligned}
\bar{D} R_{0} & =-\bar{Q} \\
\frac{1}{2}[D, \bar{D}] R_{0} & =2 i H+i\left(D \phi \frac{\delta \Gamma_{0}}{\delta \phi}-\bar{D} \bar{\phi} \frac{\delta \Gamma_{0}}{\delta \bar{\phi}}\right) \\
2 i \frac{\partial}{\partial t} R_{0} & =\{D, \bar{D}\} R_{0}=-2 i\left(D \phi \frac{\delta \Gamma_{0}}{\delta \phi}+\bar{D} \bar{\phi} \frac{\delta \Gamma_{0}}{\delta \bar{\phi}}\right) .
\end{aligned}
$$

The superconformal Ward identities involve the variance of the Lagrangian under the chiral and antichiral global Weyl scaling transformations (these are just the chiral and antichiral number operators). The combinations $\left(2 i \delta^{N} \mathcal{L}+R_{0}\right)=2 i\left(\phi K_{\phi}+\phi g_{\phi} D \phi \bar{D} \bar{\phi}\right)$ and $\left(2 i \delta^{\bar{N}} \mathcal{L}+R_{0}\right)=2 i\left(\bar{\phi} K_{\bar{\phi}}+\bar{\phi} g_{\bar{\phi}} D \phi \bar{D} \bar{\phi}\right)$ characterize the superconformal symmetry breaking. The strong requirement of superconformal symmetry implies that the theory is trivial. On the other hand, the less restrictive dilatation symmetry breaking is characterized by the sum of the above breaking terms: $\Delta_{d_{\phi}}^{D} \mathcal{L}=\frac{i d_{\phi}}{2}\left(\delta^{U} \mathcal{L}+2 R_{0}\right)=$ $-d_{\phi}\left(\left(\phi K_{\phi}+\bar{\phi} K_{\bar{\phi}}\right)+\left(\phi g_{\phi}+\bar{\phi} g_{\bar{\phi}}\right) D \phi \bar{D} \bar{\phi}\right)$. As in the case of the superconformal symmetries, the conformal Ward identity is satisfied only for a free theory. 


\section{APPENDIX A: SUPERSPACE AND SUPERCOORDINATES}

$N=1$ supersymmetry (SUSY) is generated by a complex Grassmann valued (hence nilpotent) supersymmetry charge, $Q$, its complex conjugate, $\bar{Q}$, and the Hamiltonian, $H$. Together, these charges satisfy the supersymmetry algebra

$$
\begin{gathered}
\{Q, \bar{Q}\}=2 H \\
{[Q, H]=0=[\bar{Q}, H]} \\
\{Q, Q\}=0=\{\bar{Q}, \bar{Q}\} .
\end{gathered}
$$

Since multiplying $Q$ and $\bar{Q}$ by a real phase, $Q \rightarrow e^{i \rho} Q$ and $\bar{Q} \rightarrow e^{-i \rho} \bar{Q}$, does not alter the algebra, one can define an additional charge, $R$, generating this automorphism and satisfying

$$
\begin{aligned}
& {[R, Q]=Q} \\
& {[R, \bar{Q}]=-\bar{Q}} \\
& {[R, H]=0 .}
\end{aligned}
$$

$N=1$ superspace is obtained by introducing a complex Grassmann coordinate, $\theta$, and its complex conjugate, $\bar{\theta}$, in addition to the time, $t$ so that the triplet $(t, \theta, \bar{\theta})$ denotes points in superspace. 2 The representation of the symmetry transformations as superspace differential operators on supercoordinates is most readily obtained from the motion that group multiplication induces on the parameter space of the SUSY graded Lie group. An element of this group is obtained by exponentiation of the sum of the products of the charges with the corresponding superspace point giving

$$
G(t, \theta, \bar{\theta})=e^{i(t H+\theta Q+\bar{Q} \bar{\theta})}=e^{i(\theta Q+\bar{Q} \bar{\theta})} e^{i t H}
$$

Multiplication of group elements is determined from the algebra (A.1) by exploiting the Baker-Campbell-Hausdorff formula. As a consequence of the SUSY algebra, the BakerCampbell-Hausdorff formula truncates to

$$
e^{A} e^{B}=e^{A+B+\frac{1}{2}[A, B]} .
$$

It follows that the multiplication law for group elements is given by

\footnotetext{
${ }^{2}$ For the construction of superspace and supercoordinates in extended SUSY, see [10].
} 


$$
G\left(t^{\prime}, \theta^{\prime}, \bar{\theta}^{\prime}\right) G(t, \theta, \bar{\theta})=G\left(t+t^{\prime}+i\left(\theta^{\prime} \bar{\theta}-\theta \bar{\theta}^{\prime}\right), \theta+\theta^{\prime}, \bar{\theta}+\bar{\theta}^{\prime}\right)
$$

Hence, group multiplication (from the left) induces the movement in parameter space $(t, \theta, \bar{\theta}) \rightarrow\left(t+t^{\prime}+i\left(\theta^{\prime} \bar{\theta}-\theta \bar{\theta}^{\prime}\right), \theta+\theta^{\prime}, \bar{\theta}+\bar{\theta}^{\prime}\right)$.

A supercoordinate, $\phi(t, \theta, \bar{\theta})$, whose transformation properties under $H, Q$ and $\bar{Q}$ are determined by the above shift, is defined so that

$$
\begin{aligned}
\phi(t, \theta, \bar{\theta}) & =G(t, \theta, \bar{\theta}) \phi(0,0,0) G^{-1}(t, \theta, \bar{\theta}) \\
& =e^{i(\theta Q+\bar{Q} \bar{\theta})} \phi(t, 0,0) e^{-i(\theta Q+\bar{Q} \bar{\theta})}
\end{aligned}
$$

with $\phi(t, 0,0)=e^{i t H} \phi(0,0,0) e^{-i t H}$. It then follows that

$$
G\left(t^{\prime}, \theta^{\prime}, \bar{\theta}^{\prime}\right) \phi(t, \theta, \bar{\theta}) G^{-1}\left(t^{\prime}, \theta^{\prime}, \bar{\theta}^{\prime}\right)=\phi\left(t+t^{\prime}+i\left(\theta^{\prime} \bar{\theta}-\theta \bar{\theta}^{\prime}\right), \theta+\theta^{\prime}, \bar{\theta}+\bar{\theta}^{\prime}\right) .
$$

For infinitesimal superspace parameters $(\epsilon, \eta, \bar{\eta})$, the group elements and shifted supercoordinate can be Taylor expanded about $(t, \theta, \bar{\theta})$. Employing the notation $\delta^{\mathcal{Q}} \phi$ for the superspace differential operator acting on $\phi$ corresponding to the operator $\mathcal{Q}$ so that

$$
i[\mathcal{Q}, \phi]=\delta^{\mathcal{Q}} \phi
$$

for $\mathcal{Q} \in\{H, Q, \bar{Q}\}$, and recalling that differentiation with respect to the Grassmann coordinates is given by

$$
\frac{\partial}{\partial \theta} \theta=1 \quad \frac{\partial}{\partial \bar{\theta}} \bar{\theta}=1
$$

with all other derivatives vanishing, the representation of the SUSY transformations as superspace differential operators is secured as

$$
\begin{aligned}
& i[H, \phi]=\frac{\partial}{\partial t} \phi \equiv \delta^{H} \phi \\
& i[Q, \phi]=\left(\frac{\partial}{\partial \theta}+i \bar{\theta} \frac{\partial}{\partial t}\right) \phi \equiv \delta^{Q} \phi \\
& i[\bar{Q}, \phi]=\left(-\frac{\partial}{\partial \bar{\theta}}-i \theta \frac{\partial}{\partial t}\right) \phi \equiv \delta^{\bar{Q}} \phi .
\end{aligned}
$$

From the charge algebra, $\{Q, \bar{Q}\}=2 H$, and the identity for nested commutators, $[\{Q, \bar{Q}\}, \phi]=\left\{\delta^{Q}, \delta^{\bar{Q}}\right\} \phi$, it is seen that the superspace differential operator variations $\delta^{\mathcal{Q}}$ obey the same algebra (up to a factor of $-i$ ) as the charges:

$$
\left\{\delta^{Q}, \delta^{\bar{Q}}\right\}=-2 i \delta^{H}
$$




$$
\begin{gathered}
{\left[\delta^{Q}, \delta^{H}\right]=0=\left[\delta^{\bar{Q}}, \delta^{H}\right]} \\
\left\{\delta^{Q}, \delta^{Q}\right\}=0=\left\{\delta^{\bar{Q}}, \delta^{\bar{Q}}\right\} .
\end{gathered}
$$

Since SUSY transformations induce a real time translation, $i\left(\theta^{\prime} \bar{\theta}-\theta \bar{\theta}^{\prime}\right)$ in equation (A.7), $\phi(t, \theta, \bar{\theta})$ can be chosen to be real and denoted by $\Phi$. This real supercoordinate, $\Phi^{\dagger}=\Phi$, has a component expansion

$$
\Phi(t, \theta, \bar{\theta})=x(t)+i \theta \psi(t)-i \bar{\psi}(t) \bar{\theta}+\theta \bar{\theta} W(t) .
$$

The time dependent coefficients in this decomposition consist of the even element coordinates $x(t)$ and $W(t)$ and the Grassmann odd elements $\psi(t)$ and $\bar{\psi}(t)$. The reality of $\Phi$ dictates that $x^{\dagger}=x, W^{\dagger}=W$ and $\bar{\psi}=\psi^{\dagger}$.

The $R$ symmetry variations are determined in an analogous manner using the group multiplication law

$$
e^{i \alpha R} G(t, \theta, \bar{\theta}) e^{-i \alpha R}=G\left(t, e^{i \alpha} \theta, e^{-i \alpha} \bar{\theta}\right)
$$

with $\alpha$ a real parameter. Since $R$ transformations form an Abelian subgroup, the variation of $\phi$ is given by

$$
e^{i \alpha R} \phi(t, \theta, \bar{\theta}) e^{-i \alpha R}=e^{i \alpha n_{R}} \phi\left(t, e^{i \alpha} \theta, e^{-i \alpha} \bar{\theta}\right)
$$

where $n_{R}$ is the intrinsic $R$ charge (or $R$-weight) of the $\phi$ supercoordinate. For real $\Phi$, this $R$-weight is required to vanish; $n_{R}=0$. On the other hand, for the case of complex $\phi$, which we shall consider next, $n_{R}$ is arbitrary. For infinitesimal $\alpha$, the $R$ transformations yield the $R$ variation of the supercoordinate,

$$
i[R, \phi(t, \theta, \bar{\theta})]=i\left(n_{R}+\theta \frac{\partial}{\partial \theta}-\bar{\theta} \frac{\partial}{\partial \bar{\theta}}\right) \phi(t, \theta, \bar{\theta}) \equiv \delta^{R} \phi(t, \theta, \bar{\theta}) .
$$

Once again the charge algebra $[R, Q]=Q$ and $[R, \bar{Q}]=-\bar{Q}$ implies

$$
\left[\delta^{R}, \delta^{Q}\right]=-i \delta^{Q} \quad, \quad\left[\delta^{R}, \delta^{\bar{Q}}\right]=i \delta^{\bar{Q}},
$$

as is verified by direct calculation using the explicit forms for $\delta^{R}, \delta^{Q}$, and $\delta^{\bar{Q}}$.

An alternative induced motion in parameter space can be obtained by group multiplication on the right

$$
G(t, \theta, \bar{\theta}) G\left(t^{\prime}, \theta^{\prime}, \bar{\theta}^{\prime}\right)=G\left(t+t^{\prime}-i\left(\theta^{\prime} \bar{\theta}-\theta \bar{\theta}^{\prime}\right), \theta+\theta^{\prime}, \bar{\theta}+\bar{\theta}^{\prime}\right)
$$


giving

$$
(t, \theta, \bar{\theta}) \rightarrow\left(t+t^{\prime}-i\left(\theta^{\prime} \bar{\theta}-\theta \bar{\theta}^{\prime}\right), \theta+\theta^{\prime}, \bar{\theta}+\bar{\theta}^{\prime}\right)
$$

For the supercoordinate $\varphi(t, \theta, \bar{\theta})$ defined by

$$
\varphi(t, \theta, \bar{\theta})=G^{-1}(t, \theta, \bar{\theta}) \phi(0,0,0) G(t, \theta, \bar{\theta})=\phi(-t,-\theta,-\bar{\theta})
$$

the induced motion yields

$$
G^{-1}\left(t^{\prime}, \theta^{\prime}, \bar{\theta}^{\prime}\right) \varphi(t, \theta, \bar{\theta}) G\left(t^{\prime}, \theta^{\prime}, \bar{\theta}^{\prime}\right)=\varphi\left(t+t^{\prime}-i\left(\theta^{\prime} \bar{\theta}-\theta \bar{\theta}^{\prime}\right), \theta+\theta^{\prime}, \bar{\theta}+\bar{\theta}^{\prime}\right) .
$$

For infinitesimal superspace parameters, this right multiplication induced motion leads to

$$
\begin{aligned}
-i[H, \varphi] & =\frac{\partial}{\partial t} \varphi \\
-i[Q, \varphi] & =\left(\frac{\partial}{\partial \theta}-i \bar{\theta} \frac{\partial}{\partial t}\right) \varphi \equiv D \varphi \\
-i[\bar{Q}, \varphi] & =\left(-\frac{\partial}{\partial \bar{\theta}}+i \theta \frac{\partial}{\partial t}\right) \varphi \equiv \bar{D} \varphi .
\end{aligned}
$$

where the covariant Grassmann derivatives are defined as

$$
\begin{aligned}
& D=\frac{\partial}{\partial \theta}-i \bar{\theta} \frac{\partial}{\partial t} \\
& \bar{D}=-\frac{\partial}{\partial \bar{\theta}}+i \theta \frac{\partial}{\partial t} .
\end{aligned}
$$

Note that

$$
\begin{aligned}
& \left\{\delta^{Q}, D\right\}=0=\left\{\delta^{\bar{Q}}, D\right\} \\
& \left\{\delta^{Q}, \bar{D}\right\}=0=\left\{\delta^{\bar{Q}}, \bar{D}\right\},
\end{aligned}
$$

while

$$
\begin{gathered}
\{D, \bar{D}\}=2 i \frac{\partial}{\partial t} \\
\{D, D\}=0=\{\bar{D}, \bar{D}\},
\end{gathered}
$$

(Since $\left[\partial / \partial t, \delta^{Q}\right]=0=\left[\partial / \partial t, \delta^{\bar{Q}}\right]$, it also follows that $\partial / \partial t$ is a covariant derivative).

The covariant derivatives can be used to obtain irreducible representations of the SUSY algebra by covariantly constraining the general supercoordinate $\phi$ so that either $\bar{D} \phi=0$, producing a chiral supercoordinate, or $D \phi=0$, which yields an antichiral supercoordinate. (As discussed below, $\bar{D} \phi=0$ implies that the chiral supercoordinate is necessarily complex 
and distinct from the complex antichiral supercoordinate; i.e. $\phi \neq \bar{\phi}$ ). The solution to the chiral constraint, $\bar{D} \phi=0$, is given by

$$
\phi(t, \theta, \bar{\theta})=e^{-i \theta \bar{\theta} \frac{\partial}{\partial t}} \phi(t, \theta, 0)
$$

while the antichiral constraint, $D \bar{\phi}=0$, is satisfied provided

$$
\bar{\phi}(t, \theta, \bar{\theta})=e^{i \theta \bar{\theta} \frac{\partial}{\partial t}} \bar{\phi}(t, 0, \bar{\theta})
$$

The component expansions of the chiral and antichiral supercoordinates contain complex coordinates $z, \bar{z}$ and complex (spinning) Grassmann coordinates $\xi, \bar{\xi}$ so that

$$
\begin{aligned}
& \phi(t, \theta, \bar{\theta})=e^{-i \theta \bar{\theta} \frac{\partial}{\partial t}}(z(t)+i \sqrt{2} \theta \xi(t)) \\
& \bar{\phi}(t, \theta, \bar{\theta})=e^{i \theta \bar{\theta} \frac{\partial}{\partial t}}(\bar{z}(t)-i \sqrt{2} \bar{\xi}(t) \bar{\theta}) .
\end{aligned}
$$

Alternative parametrizations of the group elements are provided by

$$
\begin{aligned}
& G_{1}(t, \theta, \bar{\theta})=e^{i(t H+\theta Q)} e^{i \bar{Q} \bar{\theta}}=e^{i \theta Q} e^{i \bar{Q} \bar{\theta}} e^{i t H} \\
& G_{2}(t, \theta, \bar{\theta})=e^{i(t H+\bar{Q} \bar{\theta})} e^{i \theta Q}=e^{i \bar{Q} \bar{\theta}} e^{i \theta Q} e^{i t H} .
\end{aligned}
$$

The relation between each parametrization is obtained using the Baker-Campbell-Hausdorff formula as

$$
G(t, \theta, \bar{\theta})=G_{1}(t-i \theta \bar{\theta}, \theta, \bar{\theta})=G_{2}(t+i \theta \bar{\theta}, \theta, \bar{\theta})
$$

The parametrizations differ by purely imaginary shifts, $\pm i \theta \bar{\theta}$, in the time coordinate. Proceeding just as before, left group multiplication with these representations also induces a motion in parameter space which now take the forms

$$
\begin{aligned}
& G_{1}\left(t^{\prime}, \theta^{\prime}, \bar{\theta}^{\prime}\right) G_{1}(t, \theta, \bar{\theta})=G_{1}\left(t+t^{\prime}-2 i \theta \bar{\theta}^{\prime}, \theta+\theta^{\prime}, \bar{\theta}+\bar{\theta}^{\prime}\right) \\
& G_{2}\left(t^{\prime}, \theta^{\prime}, \bar{\theta}^{\prime}\right) G_{2}(t, \theta, \bar{\theta})=G_{2}\left(t+t^{\prime}+2 i \theta^{\prime} \bar{\theta}, \theta+\theta^{\prime}, \bar{\theta}+\bar{\theta}^{\prime}\right) .
\end{aligned}
$$

It follows that two different supercoordinate representations can be defined using $G_{1}$ and $G_{2}$ as

$$
\phi_{1,2}(t, \theta, \bar{\theta})=G_{1,2}(t, \theta, \bar{\theta}) \phi(0,0,0) G_{1,2}^{-1}(t, \theta, \bar{\theta})
$$

Since the group elements are related by imaginary shifts in the time, so are the supercoordinate representations 


$$
\phi(t, \theta, \bar{\theta})=\phi_{1}(t-i \theta \bar{\theta}, \theta, \bar{\theta})=\phi_{2}(t+i \theta \bar{\theta}, \theta, \bar{\theta}) .
$$

$\phi$ is called the real representation while $\phi_{1}$ is known as the chiral representation and $\phi_{2}$ as the antichiral representation. The SUSY group transformations are given by the induced parameter transformations

$$
\begin{aligned}
& G_{1}\left(t^{\prime}, \theta^{\prime}, \bar{\theta}^{\prime}\right) \phi_{1}(t, \theta, \bar{\theta}) G_{1}^{-1}\left(t^{\prime}, \theta^{\prime}, \bar{\theta}^{\prime}\right)=\phi_{1}\left(t+t^{\prime}-2 i \theta \bar{\theta}^{\prime}, \theta+\theta^{\prime}, \bar{\theta}+\bar{\theta}^{\prime}\right) \\
& G_{2}\left(t^{\prime}, \theta^{\prime}, \bar{\theta}^{\prime}\right) \phi_{2}(t, \theta, \bar{\theta}) G_{2}^{-1}\left(t^{\prime}, \theta^{\prime}, \bar{\theta}^{\prime}\right)=\phi_{2}\left(t+t^{\prime}+2 i \theta^{\prime} \bar{\theta}, \theta+\theta^{\prime}, \bar{\theta}+\bar{\theta}^{\prime}\right) .
\end{aligned}
$$

Once again, for infinitesimal superspace parameters $(\epsilon, \eta, \bar{\eta})$, the SUSY variations in the chiral and antichiral representations can be determined. Using the notation $i\left[\mathcal{Q}, \phi_{1,2}\right] \equiv$ $\left(\delta^{\mathcal{Q}} \phi\right)_{1,2}$, the variations are given by

$$
\begin{array}{rlrl}
\left(\delta^{H} \phi\right)_{1} & =\frac{\partial}{\partial t} \phi_{1} \quad, \quad\left(\delta^{H} \phi\right)_{2} & =\frac{\partial}{\partial t} \phi_{2} \\
\left(\delta^{Q} \phi\right)_{1} & =\frac{\partial}{\partial \theta} \phi_{1} \quad, \quad\left(\delta^{Q} \phi\right)_{2} & =\left(\frac{\partial}{\partial \theta}+2 i \bar{\theta} \frac{\partial}{\partial t}\right) \phi_{2} \\
\left(\delta^{\bar{Q}} \phi\right)_{1} & =\left(-\frac{\partial}{\partial \bar{\theta}}+2 i \theta \frac{\partial}{\partial t}\right) \phi_{1} \quad, \quad\left(\delta^{\bar{Q}} \phi\right)_{2}=-\frac{\partial}{\partial \bar{\theta}} \phi_{2} .
\end{array}
$$

In all representations the nilpotent SUSY variations obey $\left\{\delta^{Q}, \delta^{\bar{Q}}\right\}=-2 i \frac{\partial}{\partial t}$.

Just as was the case using the real representation, the right cosets can be used to define the covariant derivatives in the chiral and antichiral representations. From the parameter space motion deduced from

$$
\begin{aligned}
& G_{1}(t, \theta, \bar{\theta}) G_{1}\left(t^{\prime}, \theta^{\prime}, \bar{\theta}^{\prime}\right)=G_{1}\left(t+t^{\prime}-2 i \theta^{\prime} \bar{\theta}, \theta+\theta^{\prime}, \bar{\theta}+\bar{\theta}^{\prime}\right) \\
& G_{2}(t, \theta, \bar{\theta}) G_{2}\left(t^{\prime}, \theta^{\prime}, \bar{\theta}^{\prime}\right)=G_{2}\left(t+t^{\prime}+2 i \theta \bar{\theta}^{\prime}, \theta+\theta^{\prime}, \bar{\theta}+\bar{\theta}^{\prime}\right),
\end{aligned}
$$

the supercoordinate defined as

$$
\varphi_{1,2}(t, \theta, \bar{\theta})=G_{1,2}^{-1}(t, \theta, \bar{\theta}) \phi(0,0,0) G_{1,2}(t, \theta, \bar{\theta})
$$

is found to satisfy

$$
\begin{aligned}
& G_{1}^{-1}\left(t^{\prime}, \theta^{\prime}, \bar{\theta}^{\prime}\right) \varphi_{1}(t, \theta, \bar{\theta}) G_{1}\left(t^{\prime}, \theta^{\prime}, \bar{\theta}^{\prime}\right)=\varphi_{1}\left(t+t^{\prime}-2 i \theta^{\prime} \bar{\theta}, \theta+\theta^{\prime}, \bar{\theta}+\bar{\theta}^{\prime}\right) \\
& G_{2}^{-1}\left(t^{\prime}, \theta^{\prime}, \bar{\theta}^{\prime}\right) \varphi_{2}(t, \theta, \bar{\theta}) G_{2}\left(t^{\prime}, \theta^{\prime}, \bar{\theta}^{\prime}\right)=\varphi_{2}\left(t+t^{\prime}+2 i \theta \bar{\theta}^{\prime}, \theta+\theta^{\prime}, \bar{\theta}+\bar{\theta}^{\prime}\right) .
\end{aligned}
$$

Finally, for infinitesimal superspace parameters $(\epsilon, \eta, \bar{\eta})$, these transformations lead to the expressions for the covariant derivatives in the chiral and antichiral representations given by 


$$
\begin{aligned}
& D_{1} \phi_{1}=\left(\frac{\partial}{\partial \theta}-2 i \bar{\theta} \frac{\partial}{\partial t}\right) \phi_{1} \quad, \quad D_{2} \phi_{2}=\frac{\partial}{\partial \theta} \phi_{2} \\
& \bar{D}_{1} \phi_{1}=-\frac{\partial}{\partial \bar{\theta}} \phi_{1} \quad, \quad \bar{D}_{2} \phi_{2}=\left(-\frac{\partial}{\partial \bar{\theta}}+2 i \theta \frac{\partial}{\partial t}\right) \phi_{2}
\end{aligned}
$$

Note that $\frac{\partial}{\partial t}$ and $\{D, \bar{D}\}=2 i \frac{\partial}{\partial t}$ are SUSY covariant derivatives in all representations.

The utility of the (anti-)chiral representations follows from the observation that when employed, the chiral and antichiral supercoordinate constraints reduce to $\bar{\theta}$ or $\theta$ independence. Thus in the chiral representation

$$
\bar{D}_{1} \phi_{1}=0=-\frac{\partial}{\partial \bar{\theta}} \phi_{1},
$$

which implies that $\phi_{1}$ is independent of $\bar{\theta}$ and can thus be expanded as

$$
\phi_{1}(t, \theta, \bar{\theta})=z(t)+i \sqrt{2} \theta \xi(t)
$$

On the other hand, in the real representation, the chiral supercoordinate is obtained from its representaion in the chiral representaion by a simple imaginary shift in time so that

$$
\begin{aligned}
\phi(t, \theta, \bar{\theta}) & =\phi_{1}(t-i \theta \bar{\theta}, \theta, \bar{\theta}) \\
& =e^{-i \theta \bar{\theta} \frac{\partial}{\partial t}}(z(t)+i \sqrt{2} \theta \xi(t)),
\end{aligned}
$$

reproducing the result presented in equation (A.27). Similarly, the antichiral constraint in the antichiral representation takes the form

$$
D_{2} \bar{\phi}_{2}=0=\frac{\partial}{\partial \theta} \bar{\phi}_{2}
$$

which implies $\bar{\phi}_{2}$ is independent of $\theta$ and thus has the component decomposition

$$
\bar{\phi}_{2}(t, \theta, \bar{\theta})=\bar{z}(t)-i \sqrt{2} \bar{\xi}(t) \bar{\theta}
$$

Once again to obtain the real representation for the antichiral supercoordinate one need only perform an imaginary time shift of the opposite sign to that used for the chiral supercoordinate. So doing, one finds

$$
\begin{aligned}
\bar{\phi}(t, \theta, \bar{\theta}) & =\bar{\phi}_{2}(t+i \theta \bar{\theta}, \theta, \bar{\theta}) \\
& =e^{i \theta \bar{\theta} \frac{\partial}{\partial t}}(\bar{z}(t)-i \sqrt{2} \bar{\xi}(t) \bar{\theta}),
\end{aligned}
$$

just as in equation (A.27). In an analogous fashion, the variations and the covariant derivatives are also related directly through imaginary time shifts as 


$$
\begin{aligned}
\delta^{Q, \bar{Q}} & =e^{-i \theta \bar{\theta} \frac{\partial}{\partial t}}\left(\delta^{Q, \bar{Q}}\right)_{1} e^{i \theta \bar{\theta} \frac{\partial}{\partial t}} \\
& =e^{i \theta \bar{\theta} \frac{\partial}{\partial t}}\left(\delta^{Q, \bar{Q}}\right)_{2} e^{-i \theta \bar{\theta} \frac{\partial}{\partial t}} \\
\stackrel{(-)}{D} & =e^{-i \theta \bar{\theta} \frac{\partial}{\partial t}} \stackrel{(-)}{D_{1}} e^{i \theta \bar{\theta} \frac{\partial}{\partial t}} \\
& =e^{i \theta \bar{\theta} \frac{\partial}{\partial t}} \stackrel{(-)}{D_{2}} e^{-i \theta \bar{\theta} \frac{\partial}{\partial t}}
\end{aligned}
$$

The SUSY transformations of the component coordinates of each supercoordinate are most easily determined in each supercoordinate's respective representation. For a chiral supercoordinate in the chiral representation, the SUSY variations are

$$
\begin{aligned}
\left(\delta^{Q} \phi\right)_{1} & =\delta^{Q} z-i \sqrt{2} \theta \delta^{Q} \xi \\
& =\frac{\partial}{\partial \theta} \phi_{1}=i \sqrt{2} \xi \\
\left(\delta^{\bar{Q}} \phi\right)_{1} & =\delta^{\bar{Q}} z-i \sqrt{2} \theta \delta^{\bar{Q}} \xi \\
& =\left(-\frac{\partial}{\partial \bar{\theta}}-2 i \theta \frac{\partial}{\partial t}\right) \phi_{1}=-2 i \theta \dot{z} .
\end{aligned}
$$

Hence the chiral supercoordinate components' SUSY transformations are

$$
\begin{array}{lrl}
\delta^{Q} z=i \sqrt{2} \xi & \quad \delta^{Q} \xi=0 \\
\delta^{\bar{Q}} z=0 \quad, & \delta^{\bar{Q}} \xi=\sqrt{2} \dot{z} .
\end{array}
$$

For an antichiral supercoordinate in the antichiral representation, the SUSY variations are

$$
\begin{aligned}
\left(\delta^{Q} \bar{\phi}\right)_{2} & =\delta^{Q} \bar{z}-i \sqrt{2} \delta^{Q} \bar{\xi} \bar{\theta} \\
& =\left(\frac{\partial}{\partial \theta}+2 i \bar{\theta} \frac{\partial}{\partial t}\right) \bar{\phi}_{2}=2 i \bar{\theta} \dot{\bar{z}} \\
\left(\delta^{\bar{Q}} \bar{\phi}\right)_{2} & =\delta^{\bar{Q}} \bar{z}-i \sqrt{2} \delta^{\bar{Q}} \bar{\xi} \bar{\theta} \\
& =-\frac{\partial}{\partial \bar{\theta}} \bar{\phi}_{2}=-i \sqrt{2} \bar{\xi}
\end{aligned}
$$

Hence the antichiral supercoordinate components' SUSY transformations are

$$
\begin{array}{ll}
\delta^{Q} \bar{z}=0 & , \quad \delta^{Q} \bar{\xi}=-\sqrt{2} \dot{\bar{z}} \\
\delta^{\bar{Q}} \bar{z}=-i \sqrt{2} \bar{\xi} & , \quad \delta^{\bar{Q}} \bar{\xi}=0 .
\end{array}
$$

Finally for the real supercoordinate in the real representation, the SUSY variations are

$$
\begin{aligned}
\delta^{Q} \Phi & =\delta^{Q} x-i \theta \delta^{Q} \psi-i \delta^{Q} \bar{\psi} \bar{\theta}+\theta \bar{\theta} \delta^{Q} W \\
& \left.=\left(\frac{\partial}{\partial \theta}+i \bar{\theta} \frac{\partial}{\partial t}\right) \Phi=i \psi+\bar{\theta}(W+i \dot{(} x)\right)+\theta \bar{\theta} \dot{\psi}
\end{aligned}
$$




$$
\begin{aligned}
\delta^{\bar{Q}} \phi & =\delta^{\bar{Q}} x-i \theta \delta^{\bar{Q}} \psi-i \delta^{\bar{Q}} \bar{\psi} \bar{\theta}+\theta \bar{\theta} \delta^{\bar{Q}} W \\
& =\left(-\frac{\partial}{\partial \bar{\theta}}-i \theta \frac{\partial}{\partial t}\right) \phi=-i \bar{\psi}+\theta(W-i(x))+\theta \bar{\theta} \dot{\bar{\psi}}
\end{aligned}
$$

Hence the real supercoordinate components' SUSY transformations are

$$
\begin{array}{rlrl}
\delta^{Q} x & =i \psi & & \delta^{Q} W=\frac{\partial}{\partial t} \psi \\
\delta^{Q} \psi & =0 & , & \delta^{Q} \bar{\psi}=i(W+i \dot{x}) \\
\delta^{\bar{Q}} x & =-i \bar{\psi} \quad, & \delta^{\bar{Q}} W=\frac{\partial}{\partial t} \bar{\psi} \\
\delta^{\bar{Q}} \psi & =i(W-i \dot{x}) & , & \delta^{\bar{Q}} \bar{\psi}=0 .
\end{array}
$$

The last $(\theta \bar{\theta})$ component of a real supercoordinate transforms as a total time derivative, $\delta^{Q} W=\frac{\partial}{\partial t} \psi$ and $\delta^{\bar{Q}} W=\frac{\partial}{\partial t} \bar{\psi}$. Hence, assuming total differentials in time integrate to zero, it follows that $\int d t W$ is SUSY invariant; $\delta^{Q} \int d t W=0=\delta^{\bar{Q}} \int d t W$. In the superspace framework, to extract the last component of the real supercoordinate, one integrates over a vector measure defined as

$$
\int d V \Phi=\int d t d \theta d \bar{\theta} \Phi=-\int d t W
$$

In obtaining this result, one employs the convention that integration over the Grassmann parameters is defined just as differentiation so that

$$
\begin{aligned}
& d \theta=\frac{\partial}{\partial \theta} \\
& d \bar{\theta}=\frac{\partial}{\partial \bar{\theta}}
\end{aligned}
$$

Since $\delta^{Q} \int d t W=\delta^{\bar{Q}} \int d t W=0$, it follows that $\delta^{Q} \int d V \Phi=\delta^{\bar{Q}} \int d V \Phi=0$ and hence is SUSY invariant.

For chiral, $\chi$, and antichiral, $\bar{\chi}$, Grassmann supercoordinates, the last component of each also transforms as a total derivative or is itself invariant. Thus additional SUSY invariant terms can be secured as integrals of a (anti-)chiral Grassmann supercoordinate taken over a (anti-)chiral measure defined as $\int d S \chi=\int d t d \theta \chi$ and $\int d \bar{S} \bar{\chi}=\int d t d \bar{\theta} \bar{\chi}$. 


\section{APPENDIX B: SUPERCONFORMAL TRANSFORMATIONS}

The $N=1$ superconformal group algebra can be obtained using the product rules of the orthosymplectic group $\operatorname{OSp}(2,1)$ whose algebra consists of 8 (charges) generators [11]. Three charges generate the familiar conformal subgroup $S O(2,1)$ of quantum mechanics. They consist of the Hamiltonian $H$ which generates time translations, the time dilatation charge $D$ and the conformal transformation charge $K$. The remaining 5 charges consist of the generator, $R$, of superspace Grassmann (spinning) coordinate rotations, the anticommuting SUSY charges, $Q$ and $\bar{Q}$, and the anticommuting special (conformal) SUSY charges, $S$ and $\bar{S}$. An element of $O S p(2,1)$ can be represented by matrices having the generic structure 12

$$
\left(\begin{array}{cc|c}
S O(2,1) & Q \\
H, & D, K & S \\
\hline \bar{Q} & \bar{S} & R
\end{array}\right)
$$

By examination of the matrix commutators, the $O S p(2,1)$ algebra can be extracted as

$$
\begin{array}{rlrl}
{[H, D]} & =i H & {[H, K]=2 i D} & {[D, K]=i K} \\
\{Q, \bar{Q}\} & =2 H & \{S, \bar{S}\} & =2 K \\
{[R, Q]} & =Q & {[R, S]} & =-S \\
{[R, \bar{Q}]} & =-\bar{Q} & {[R, \bar{S}]} & =\bar{S} \\
{[D, Q]} & =-\frac{i}{2} Q & {[D, \bar{Q}]} & =-\frac{i}{2} \bar{Q} \\
{[D, S]} & =\frac{i}{2} S & {[D, \bar{S}]=\frac{i}{2} \bar{S}} \\
{[K, Q]} & =i \bar{S} & {[K, \bar{Q}]=i S} \\
{[H, S]} & =-i \bar{Q} & {[H, \bar{S}]} & =-i Q \\
\{Q, S\} & =-2 D-i R & \{\bar{Q}, \bar{S}\} & =-2 D+i R
\end{array}
$$

with all other (anti-)commutators vanishing.

The representation of these transformations on a general supercoordinate $\phi$ can be determined by the techniques of Appendix A. Employing the notation $i[\mathcal{Q}, \phi]=$ $\delta^{\mathcal{Q}} \phi$ for each charge $\mathcal{Q}$, the variations in the real representation are given by 


\begin{tabular}{|l|l|}
\hline Symmetry & Transformation \\
\hline \hline Time Translation & $\delta^{H} \phi(t, \theta, \bar{\theta})=\frac{\partial}{\partial t} \phi(t, \theta, \bar{\theta})$ \\
\hline Supersymmetry & $\delta^{Q} \phi(t, \theta, \bar{\theta})=\left(\frac{\partial}{\partial \theta}+i \bar{\theta} \frac{\partial}{\partial t}\right) \phi(t, \theta, \bar{\theta})$ \\
\cline { 2 - 3 } & $\delta^{\bar{Q}} \phi(t, \theta, \bar{\theta})=\left(-\frac{\partial}{\partial \theta}-i \theta \frac{\partial}{\partial t}\right) \phi(t, \theta, \bar{\theta})$ \\
\hline \multirow{2}{*}{$R$ Transformation } & $\delta^{R} \phi(t, \theta, \bar{\theta})=i\left(n_{\phi}+\theta \frac{\partial}{\partial \theta}-\bar{\theta} \frac{\partial}{\partial \bar{\theta}}\right) \phi(t, \theta, \bar{\theta})$ \\
\hline \multirow{2}{*}{ Dilatation } & $\delta^{D} \phi(t, \theta, \bar{\theta})=\left(d_{\phi}+t \frac{\partial}{\partial t}+\frac{1}{2} \theta \frac{\partial}{\partial \theta}+\frac{1}{2} \bar{\theta} \frac{\partial}{\partial \theta}\right) \phi(t, \theta, \bar{\theta})$ \\
\hline Conformal & $\delta^{K} \phi(t, \theta, \bar{\theta})=\left(2 t d_{\phi}+i n_{\phi} \theta \bar{\theta}+t\left(\theta \frac{\partial}{\partial \theta}+\bar{\theta} \frac{\partial}{\partial \theta}\right)+t^{2} \frac{\partial}{\partial t}\right) \phi(t, \theta, \bar{\theta})$ \\
\hline Superconformal & $\delta^{S} \phi(t, \theta, \bar{\theta})=\left(i \theta\left(2 d_{\phi}-n_{\phi}\right)+t \frac{\partial}{\partial \theta}+i t \theta \frac{\partial}{\partial t}+i \theta \bar{\theta} \frac{\partial}{\partial \theta}\right) \phi(t, \theta, \bar{\theta})$ \\
\cline { 2 - 3 } & $\delta^{\bar{S}} \phi(t, \theta, \bar{\theta})=\left(-i \bar{\theta}\left(2 d_{\phi}+n_{\phi}\right)-t \frac{\partial}{\partial \theta}-i t \bar{\theta} \frac{\partial}{\partial t}+i \theta \bar{\theta} \frac{\partial}{\partial \theta}\right) \phi(t, \theta, \bar{\theta})$ \\
\hline
\end{tabular}

where $n_{\phi}$ is the $R$ charge (weight) of the supercoordinate while $d_{\phi}$ is its scaling weight (engineering dimension). If $\phi$ is hermitean, then $n_{\phi}=0$, while for chiral $\phi$ with $R$-weight $n_{\phi}$, then the antichiral supercoordinate $\bar{\phi}$ has $R$-weight $n_{\bar{\phi}}=-n_{\phi}$. Requiring chiral consistency in the commutation relations of the covariant derivatives, $D$ and $\bar{D}$, with the transformations imply that for chiral supercoordinates $n_{\phi}=-2 d_{\phi}$, while for antichiral supercoordinates that $n_{\bar{\phi}}=2 d_{\phi}$. As can be explicitly demonstrated, the variations obey the superconformal algebra. Thus if $\left[\mathcal{Q}_{1}, \mathcal{Q}_{2}\right]_{ \pm}=$if $\mathcal{Q}_{3}$, then $\left[\delta^{\mathcal{Q}_{1}}, \delta^{\mathcal{Q}_{2}}\right]_{ \pm}=f \delta^{\mathcal{Q}_{3}}$.

In terms of the component coordinates, the superconformal and $U(1)$ transformations are given by 


\begin{tabular}{|c|c|c|}
\hline Symmetry & $\begin{array}{l}\text { Chiral Components } \\
\text { Transformation }\end{array}$ & $\begin{array}{l}\text { Antichiral Components } \\
\text { Transformation }\end{array}$ \\
\hline \multirow[t]{2}{*}{ Time Translation } & $\delta^{H} z=\dot{z}$ & $\delta^{H} \bar{z}=\dot{\bar{z}}$ \\
\hline & $\delta^{H} \xi=\dot{\xi}$ & $\delta^{H} \bar{\xi}=\dot{\bar{\xi}}$ \\
\hline \multirow[t]{4}{*}{ Supersymmetry } & $\delta^{Q} z=i \sqrt{2} \xi$ & $\delta^{Q} \bar{z}=0$ \\
\hline & $\delta^{Q} \xi=0$ & $\delta^{Q} \bar{\xi}=-\sqrt{2} \dot{\bar{z}}$ \\
\hline & $\delta^{\bar{Q}} z=0$ & $\delta^{\bar{Q}} \bar{z}=-\bar{\xi}$ \\
\hline & $\delta^{\bar{Q}} \xi=+\sqrt{2} \dot{z}$ & $\delta^{\bar{Q}} \bar{\xi}=0$ \\
\hline \multirow[t]{2}{*}{$R_{0}$ Transformation } & $\delta^{R_{0}} z=0$ & $\delta^{R_{0}} \bar{z}=0$ \\
\hline & $\delta^{R_{0}} \xi=+i \xi$ & $\delta^{R_{0}} \bar{\xi}=-i \bar{\xi}$ \\
\hline \multirow[t]{2}{*}{ Dilatation } & $\delta^{D} z=\left(d+t \partial_{t}\right) z$ & $\delta^{D} \bar{z}=\left(d+t \partial_{t}\right) \bar{z}$ \\
\hline & $\delta^{D} \xi=\left(d_{\xi}+t \partial_{t}\right) \xi$ & $\delta^{D} \bar{\xi}=\left(d_{\xi}+t \partial_{t}\right) \bar{\xi}$ \\
\hline \multirow[t]{2}{*}{ Conformal } & $\delta^{K} z=\left(2 t d+t^{2} \partial_{t}\right) z$ & $\delta^{K} \bar{z}=\left(2 t d+t^{2} \partial_{t}\right) \bar{z}$ \\
\hline & $\delta^{K} \xi=\left(2 t d_{\xi}+t^{2} \partial_{t}\right) \xi$ & $\delta^{K} \bar{\xi}=\left(2 t d_{\xi}+t^{2} \partial_{t}\right) \bar{\xi}$ \\
\hline \multirow[t]{4}{*}{ Superconformal } & $\delta^{S} z=0$ & $\delta^{S} \bar{z}=i t \sqrt{2} \bar{\xi}$ \\
\hline & $\delta^{S} \xi=-\sqrt{2}\left[2\left(d_{\xi}-\frac{1}{2}\right)+t \partial_{t}\right] z$ & $\delta^{S} \bar{\xi}=0$ \\
\hline & $\delta^{\bar{S}} z=-i t \sqrt{2} \xi$ & $\delta^{\bar{S}} \bar{z}=0$ \\
\hline & $\delta^{\bar{S}} \xi=0$ & $\delta^{\bar{S}} \bar{\xi}=+\sqrt{2}\left[2\left(d_{\xi}-\frac{1}{2}\right)+t \partial_{t}\right] \bar{z}$ \\
\hline \multirow[t]{2}{*}{$U_{J}(1)$} & $\delta^{J} z=+i z$ & $\delta^{J} \bar{z}=-i \bar{z}$ \\
\hline & $\delta^{J} \xi=+i \xi$ & $\delta^{J} \bar{\xi}=-i \bar{\xi}$ \\
\hline
\end{tabular}

Note that in order for the superconformal algebra to close, it is necessary that $d_{\xi}=d_{\phi}+\frac{1}{2}$.

Some of the superspace dependent charges of the superconformal algebra have explicit time dependence and thus do not transform as supercoordinates under SUSY transformations. It is possible, however, to add explicit $\theta$ and $\bar{\theta}$ terms to these charges such that the new charge transforms under a restricted SUSY transformation as would a time independent supercoordinate while still obeying the same superconformal algebra as the associated 
charge without the explicit $\theta$ and $\bar{\theta}$. Such a quasi-supercoordinate $\hat{\mathcal{Q}}$ is defined so that under a SUSY transformation parametrized with the superspace coordinates $\theta$ and $\bar{\theta}$, it transforms as would a supercoordinate so that [8]

$$
i[\theta Q+\bar{Q} \bar{\theta}, \hat{\mathcal{Q}}]=\left(\theta \frac{\partial}{\partial \theta}+\bar{\theta} \frac{\partial}{\partial \bar{\theta}}\right) \hat{\mathcal{Q}} .
$$

The solution to this equation is given by

$$
\hat{\mathcal{Q}}(\theta, \bar{\theta})=e^{i(\theta Q+\bar{Q} \bar{\theta})} \mathcal{Q} e^{-i(\theta Q+\bar{Q} \bar{\theta})} .
$$

For each of the superconformal charges the corresponding quasi-supercoordinate is constructed

$$
\begin{aligned}
\hat{H} & =H \\
\hat{Q} & =Q-2 i \bar{\theta} H \\
\hat{\bar{Q}} & =\bar{Q}+2 i \theta H \\
\hat{R} & =R-i \theta Q+i \bar{Q} \bar{\theta}-2 \theta \bar{\theta} H \\
\hat{D} & =D-\frac{1}{2} \theta Q-\frac{1}{2} \bar{Q} \bar{\theta} \\
\hat{K} & =K+\theta \bar{S}+S \bar{\theta}+\theta \bar{\theta} R \\
\hat{S} & =S+\theta(-2 i D+R)-i \theta \bar{\theta} \bar{Q} \\
\hat{\bar{S}} & =\bar{S}+\bar{\theta}(+2 i D+R)+i \theta \bar{\theta} Q .
\end{aligned}
$$

The quasi-supercoordinates of charges $\hat{\mathcal{Q}}$ obey the same superconformal algebra as the $\mathcal{Q}$. 


\section{APPENDIX C: QUANTUM ACTION PRINCIPLE}

The generating functional, $Z[\eta, \bar{\eta}]$, for ground state expectation values of the time ordered products of chiral and antichiral supercoordinates is given by the Feynman path integral

$$
\begin{aligned}
Z[\eta, \bar{\eta}] & =<0\left|T e^{i \int d S \eta \phi+i \int d \bar{S} \bar{\eta} \bar{\phi}}\right| 0> \\
& =\int[d \phi][d \bar{\phi}] e^{i\left(\Gamma_{0}+\int d S \eta \phi+\int d \bar{S} \bar{\eta} \bar{\phi}\right)}
\end{aligned}
$$

where $\Gamma_{0}=\int d V \mathcal{L}=\int d t L$ is the classical action. For the model discussed in the body of this paper, the action is given in equation (2.2) or equivalently equation (2.3). Here $\eta$ and $\bar{\eta}$ are chiral and antichiral Grassmann scalar supercoordinate sources having the component decompositions

$$
\begin{aligned}
& \eta(t, \theta, \bar{\theta})=e^{-i \theta \bar{\theta} \frac{\partial}{\partial t}}\left(\frac{i}{\sqrt{2}} \chi(t)+\theta J(t)\right) \\
& \bar{\eta}(t, \theta, \bar{\theta})=e^{i \theta \bar{\theta} \frac{\partial}{\partial t}}\left(\frac{i}{\sqrt{2}} \bar{\chi}(t)+\bar{\theta} \bar{J}(t)\right) .
\end{aligned}
$$

Using the chiral and antichiral measures, $d S=d t \frac{\partial}{\partial \theta}$, and $d \bar{S}=d t \frac{\partial}{\partial \theta}$ respectively, the generating functional expressed in terms of the component coordinates is

$$
Z[\eta, \bar{\eta}]=Z[J, \bar{J}, \chi, \bar{\chi}]=\int[d z][d \bar{z}][d \xi][d \bar{\xi}] e^{i \int d t\{L+J z+\bar{J} \bar{z}+\chi \xi+\bar{\chi} \bar{\xi}\}}
$$

The connected function generating functional, $Z^{c}[\eta, \bar{\eta}]$, is defined as

$$
Z[\eta, \bar{\eta}]=e^{Z^{c}[\eta, \bar{\eta}]}
$$

The quantum effective action $\Gamma[\phi, \bar{\phi}]$, which is the generating functional for one-particle irreducible (1-PI) functions is defined as the Legendre transform of $Z^{c}$ :

$$
\Gamma[\phi, \bar{\phi}]=Z^{c}[\eta, \bar{\eta}]-i \int d S \eta \phi-i \int d \bar{S} \bar{\eta} \bar{\phi}
$$

where the sources for the 1-PI functions are the classical supercoordinates $\phi$ and $\bar{\phi}$, defined as

$$
\frac{1}{i} \frac{\delta Z^{c}}{\delta \eta}=\phi \quad, \quad \frac{1}{i} \frac{\delta Z^{c}}{\delta \bar{\eta}}=\bar{\phi}
$$

In terms of the component coordinates, the Legendre transform is

$$
\Gamma[z, \bar{z}, \xi, \bar{\xi}]=Z^{c}[J, \bar{J}, \chi, \bar{\chi}]-i \int d t(J z+\bar{J} \bar{z}+\chi \xi+\bar{\chi} \bar{\xi})
$$


with the classical supercoordinates given by

$$
\begin{array}{lll}
\frac{1}{i} \frac{\delta Z^{c}}{\delta J}=z & , & \frac{1}{i} \frac{\delta Z^{c}}{\delta \bar{J}}=\bar{z} \\
\frac{1}{i} \frac{\delta Z^{c}}{\delta \chi}=\xi & & \frac{1}{i} \frac{\delta Z^{c}}{\delta \bar{\chi}}=\bar{\xi}
\end{array}
$$

It should be noted that in the supercoordinate case, the chiral supercoordinate functional derivatives are Grassmann odd. Thus they have been used in the Grassmann odd chiral and antichiral integrals in order to give chiral, $\delta^{S}$, and antichiral, $\delta^{\bar{S}}$, superspace delta functions that then integrate to Grassmann even functions so that

$$
\begin{aligned}
\frac{\delta \phi(1)}{\delta \phi(2)} & =\delta^{S}(1,2)=\bar{D}_{1} \delta(1,2) \\
& =\theta_{12} e^{-i \theta_{2} \bar{\theta}_{12} \frac{\partial}{\partial t_{1}}} \delta\left(t_{1}-t_{2}\right) \\
\frac{\delta \bar{\phi}(1)}{\delta \bar{\phi}(2)} & =\delta^{\bar{S}}(1,2)=D_{1} \delta(1,2) \\
& =\bar{\theta}_{12} e^{i \theta_{12} \bar{\theta}_{2} \frac{\partial}{\partial t_{1}}} \delta\left(t_{1}-t_{2}\right)
\end{aligned}
$$

where the real measure delta function is defined as $\delta(1,2)=\delta\left(t_{1}-t_{2}\right) \theta_{12} \bar{\theta}_{12}$ with $\theta_{12}=$ $\theta_{1}-\theta_{2} ; \bar{\theta}_{12}=\bar{\theta}_{1}-\bar{\theta}_{2}$.

The supercoordinate Euler-Lagrange equations for the above chiral supercoordinate model with action (2.2) can be derived either graphically or by using a formal change of integration variable in the path integral. So doing, one finds that, for this action, no renormalization is needed. Consequently, the equations of motion for the Green's function generating functional are

$$
\frac{\delta \Gamma_{0}}{\delta \phi(1)} Z[\eta, \bar{\eta}]=-\eta(1) Z[\eta, \bar{\eta}] \quad, \quad \frac{\delta \Gamma_{0}}{\delta \bar{\phi}(1)} Z[\eta, \bar{\eta}]=-\bar{\eta}(1) Z[\eta, \bar{\eta}]
$$

while for connected functions one finds

$$
\frac{\delta \Gamma_{0}}{\delta \phi(1)} Z^{c}[\eta, \bar{\eta}]=-\eta(1) \quad, \quad \frac{\delta \Gamma_{0}}{\delta \bar{\phi}(1)} Z^{c}[\eta, \bar{\eta}]=-\bar{\eta}(1)
$$

The functional derivatives of the classical action, $\Gamma_{0}$, are just the supercoordinate generalization of the classical Euler-Lagrange derivatives of the Lagrangian. For the action under consideration, $\Gamma_{0}=\int d V[g D \phi \bar{D} \bar{\phi}+K] \equiv \int d V \mathcal{L}$, the Euler-Lagrange derivatives are

$$
\begin{aligned}
\frac{\delta \Gamma_{0}}{\delta \phi(1)} & =-\bar{D}\left(\frac{\partial \mathcal{L}}{\partial \phi}-D\left(\frac{\partial \mathcal{L}}{\partial D \phi}\right)\right) \\
& =\bar{D}\left(g D \bar{D} \bar{\phi}-K_{\phi}\right)
\end{aligned}
$$




$$
\begin{aligned}
\frac{\delta \Gamma_{0}}{\delta \bar{\phi}(1)} & =-D\left(\frac{\partial \mathcal{L}}{\partial \bar{\phi}}-\bar{D}\left(\frac{\partial \mathcal{L}}{\partial \bar{D} \bar{\phi}}\right)\right) \\
& =-D\left(g \bar{D} D \phi+K_{\bar{\phi}}\right)
\end{aligned}
$$

The operator inserted Euler-Lagrange equations can then be Legendre transformed in order to obtain the effective action equations of motion

$$
\left[i \frac{\delta \Gamma_{0}}{\delta \phi(1)}\right] \Gamma[\phi, \bar{\phi}]=\frac{\delta \Gamma[\phi, \bar{\phi}]}{\delta \phi(1)} \quad, \quad\left[i \frac{\delta \Gamma_{0}}{\delta \bar{\phi}(1)}\right] \Gamma[\phi, \bar{\phi}]=\frac{\delta \Gamma[\phi, \bar{\phi}]}{\delta \bar{\phi}(1)}
$$

In a similar fashion, using either graphical or path integral techniques, composite operator, $\mathcal{O}$, inserted Euler-Lagrange equations of motion can be gleaned and take the form

$$
\left[i \mathcal{O} \frac{\delta \Gamma_{0}}{\delta \tilde{\phi}(1)}\right] \Gamma[\phi, \bar{\phi}]=\frac{\delta}{\delta \tilde{\phi}(1)}(\mathcal{O} \Gamma[\phi, \bar{\phi}])
$$

where $\tilde{\phi}$ is either $\phi$ or $\bar{\phi}$. Thus the quantum action principle relates the variation of the effective action to that of the classical action

$$
\begin{aligned}
\delta \Gamma & =\left(\int d S \delta \phi \frac{\delta}{\delta \phi}+\int d \bar{S} \delta \bar{\phi} \frac{\delta}{\delta \bar{\phi}}\right) \Gamma \\
& =\left[i \delta \Gamma_{0}\right] \Gamma \\
& =\left[i \int d S \delta \phi \frac{\delta \Gamma_{0}}{\delta \phi}+i \int d \bar{S} \delta \bar{\phi} \frac{\delta \Gamma_{0}}{\delta \bar{\phi}}\right] \Gamma \\
& =\left[i \int d V \delta \mathcal{L}\right] \Gamma,
\end{aligned}
$$

where $\delta \phi$ and $\delta \bar{\phi}$ are chiral and antichiral products of the supercoordinates and their derivatives. 


\section{APPENDIX D: ANTICOMMUTING GRASSMANN (SPINNING) COORDINATE DETERMINANT}

In this appendix, we shall explicitly integrate the anticommuting Grassmann (spinning) coordinate out of the path integral representation of the Green function generating functional resulting in a path integral involving only the particle ordinary spatial coordinates. Expressed in terms of the component coordinates, including the anticommuting Grassmann (spinning) coordinates, the path integral is given by

$$
Z[J, \bar{J}, \chi, \bar{\chi}]=\int[d z][d \bar{z}][d \xi][d \bar{\xi}] e^{i \int d t\{L+J z+\bar{J} \bar{z}+\chi \xi+\bar{\chi} \bar{\xi}\}}
$$

where the Lagrangian with canonically normalized kinetic energy term so $g=\frac{1}{4}$ is

$$
L=\dot{z} \dot{\bar{z}}+\frac{i}{2}(\xi \dot{\bar{\xi}}-\dot{\xi} \bar{\xi})+i\left(K_{z} \dot{z}-K_{\bar{z}} \dot{\bar{z}}\right)-2 K_{z \bar{z}} \xi \bar{\xi}
$$

Since this Lagrangian is bilinear in the Grassmann (spinning) coordinates, they can be integrated out as 13] 14

$$
\int[d \xi][d \bar{\xi}] e^{i \int d t\left[L_{f}+\chi \xi+\bar{\chi} \bar{\xi}\right]}=\frac{\operatorname{Det}\left[S_{F}^{-1}\right]}{\operatorname{Det}\left[S_{F 0}^{-1}\right]} e^{i \int d t d t^{\prime} \bar{\chi}(t) S_{F}\left(t-t^{\prime}\right) \chi\left(t^{\prime}\right)},
$$

where

$$
L_{f}=\frac{i}{2}(\xi \dot{\bar{\xi}}-\dot{\xi} \bar{\xi})-2 K_{z \bar{z}} \xi \bar{\xi}
$$

is that part of the Lagrangian having dependence on the anticommuting (spinning) coordinates $\xi$ and $\bar{\xi}$. Here we normalized the determinant by its noninteracting $\left(K_{z \bar{z}}=0\right.$ value so that the path integral gives unity in the absence of all interactions and sources. The inverse fermion propagator, determined from $\mathcal{L}_{f}$, is

$$
S_{F}^{-1}\left(t-t^{\prime} ; z, \bar{z}\right)=\left(i \frac{d}{d t}-2 K_{z \bar{z}}+i \epsilon\right) \delta\left(t-t^{\prime}\right),
$$

while $S_{F 0}^{-1}=\left(i \frac{d}{d t}+i \epsilon\right) \delta\left(t-t^{\prime}\right)$. The determinant can be secured as a product of the eigenvalues, $\lambda$, of $S_{F}^{-1}$ defined through the eigenvalue equation

$$
\int d t^{\prime} S_{F}^{-1}\left(t-t^{\prime} ; z, \bar{z}\right) \psi_{\lambda}\left(t^{\prime} ; z, \bar{z}\right)=\lambda \psi_{\lambda}(t ; z, \bar{z})
$$

The solution to this integral equation is given by

$$
\psi_{\lambda}(t ; z, \bar{z})=\psi_{\lambda}\left(t_{0} ; z, \bar{z}\right) e^{-i \lambda\left(t-t_{0}\right)-2 i \int_{t_{0}}^{t} d \tau K_{z \bar{z}}(z, \bar{z})}
$$


Eventually, we will want to take the adiabatic limit, $t \rightarrow \infty$. Since the time integral in the exponential diverges in this limit, we must regulate the integral in the intermediate steps. We do so by introducing a cutoff at time $T$. As such, we need to impose boundary conditions on the solution. Imposing anti-periodic boundary conditions, the anticommuting coordinate eigenfunctions are then required to satisfy

$$
\psi_{\lambda}(-T)=-\psi_{\lambda}(T)
$$

This, in turn, restricts the allowed eigenvalues to be

$$
\lambda \rightarrow \lambda_{n}=\frac{(2 n+1) \pi}{2 T}-\frac{1}{T} \int_{-T}^{T} d \tau K_{z \bar{z}}
$$

where $n=0, \pm 1, \pm 2, \ldots$ is any integer.

The desired ratio of determinants is then simply given by the product of the eigenvalues as

$$
\begin{aligned}
\frac{\operatorname{Det}\left[S_{F}^{-1}\right]}{\operatorname{Det}\left[S_{F 0}^{-1}\right]} & =\frac{\prod_{n=-\infty}^{\infty} \lambda_{n}}{\left.\prod_{n=-\infty}^{\infty} \lambda_{n}\right|_{K_{z \bar{z}}=0}} \\
& =\prod_{n=-\infty}^{\infty}\left[1-\frac{\int_{-T}^{T} d \tau 2 K_{z \bar{z}}}{(2 n+1) \pi}\right] \\
& =\cos \left[\int_{-T}^{T} d \tau K_{z \bar{z}}\right] .
\end{aligned}
$$

Taking the adiabatic limit, $T \rightarrow \infty$, Green function generating functional (ignoring the Grassmann sources for simplicity) takes the form

$$
Z[J, \bar{J}, 0,0]=\int[d z][d \bar{z}]\left(e^{i \int d t K_{z \bar{z}}(z, \bar{z})}+e^{-i \int d t K_{z \bar{z}}(z, \bar{z})}\right) e^{i \int d t\left[\dot{z} \dot{\bar{z}}+i\left(K_{z} \dot{z}-K_{\bar{z}} \dot{\bar{z}}\right)+J z+\bar{J} \bar{z}\right]} .
$$

Finally, letting $z=\sqrt{\frac{m}{2}}(x+i y), \bar{z}=\sqrt{\frac{m}{2}}(x-i y)$, and scaling $K \rightarrow q K$, with $q$ the particle electric cahrge, so that $K_{z \bar{z}}=-\frac{q}{2 m} B(x, y)=-\frac{q}{2 m} \epsilon_{i j} \partial_{i} A_{j}(x, y)$, where $B(x, y)$ is the magnetic field and $\vec{A}$ is the vector potential, we secure the path integral representation

$$
Z\left[J_{x}, J_{y}, 0,0\right]=\int[d x][d y]\left(e^{i \int d t \frac{q B(x, y)}{2 m}}+e^{-i \int d t \frac{q B(x, y)}{2 m}}\right) e^{i \int d t\left[\frac{1}{2} m \vec{v}^{2}+q \vec{A} \cdot \vec{v}+J_{x} x+J_{y} y\right]} .
$$

Here $\vec{v}=\hat{e}_{1} \dot{x}+\hat{e}_{2} \dot{y}$ is the particle velocity and we have replaced the complex sources $J, \bar{J}$ with the real sources $J_{x}, J_{y}$. This path integral expression is completely equivalent (note in this appendix, we have set $\hbar=1$ ) to the path integral resulting from the Lagrangian (2.11) or the Hamiltonian (2.12) where the trace over the Pauli matrix magnetic dipole moment interaction Hamiltonian has been taken to obtain the two magnetic field exponential terms in the path integral. 
This work was supported in part by the U.S. Department of Energy under grant DE-FG0291ER40681 (Task B). 


\section{REFERENCES}

[1] E. Witten, Nucl. Phys. B185 (1981) 513.

[2] for a reviews of 1-dimensional supersymmetric quantum mechanics, see, for example, F. Cooper, A. Khare, and U. Sukhatme, Phys.Rept. 251, 267 (1995) and W. Kwong and J.L. Rosner, Prog. Theor. Phys. Suppl. 86 (1986) 366, (Festschrift volume in honor of Y. Nambu).

[3] T.E. Clark, S.T. Love and S.R. Nowling, Mod. Phys. Lett. A15 (2000) 2105.

[4] M. de Crombrugghe and V. Rittenberg, Ann. Phys. 151, 99 (1983); A. Barducci, R. Casalbuoni and L. Lusanna, Nuovo Cimento 35A, 377 (1976).

[5] R. Jackiw, Phys. Rev. D 29, 2375 (1984).

[6] R.J. Hughes, V.A. Kostelecký and M.M Nieto, Phys. Rev. D 34, 1100 (1986); V.A. Kostelecký, V.I. Man'ko, M.M. Nieto and D.R. Truax, Phys. Rev. A 48, 951 (1993).

[7] R. Jackiw, Delta function potentials in two-dimensional and three-dimensional quantum mechanics, Beg Memorial Volume, edited by A. Ali and P. Hoodbhoy, (World Scientific, Singapore 1991).

[8] T.E. Clark, O. Piguet and K. Sibold, Nucl. Phys. B143, 445 (1978), Nucl. Phys. B169, 77 (1980), Nucl. Phys. B172, 201 (1980).

[9] T.E. Clark and S.T. Love, Int. Jour. of Mod. Phys. A11, 2807 (1996).

[10] C.M. Hull, The Geometry of Supersymmetric Quantum Mechanics hep-th/9910028.

[11] E. D'Hoker and L. Vinet, Lett. in Math. Phys. 8, 439 (1984), Commun. Math. Phys. 97, 391 (1985).

[12] C. Duval and P.A. Horváthy, J. Math. Phys. 35, 2516 (1994).

[13] E. Gildener and A. Patrascioiu, Phys. Rev. D16, 1802 (1977).

[14] F. Cooper and B. Freedman, Ann. of Phys. 146, 262 (1983). 